\title{
ARL AnNual Salary Survey 2008-2009
}

Compiled and Edited by

Martha KyRILLIDOU

Les BlAND

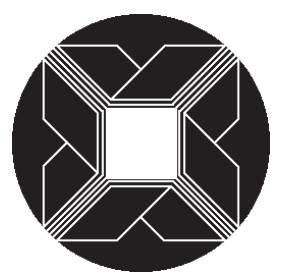

Association of ResEARCh LibraRIES

WASHINGTON, DC 
ARL Annual Salary Survey 2008-2009

The quantitative tables presented in this publication are not indicative of performance and outcomes and should not be used as measures of library quality. In comparing any individual library to ARL medians or to other ARL members, one must be careful to make such comparisons within the context of differing institutional and local goals and characteristics.

Custom reports based on the Salary Survey data are also available. Contact the ARL Statistics and Measurement Program Officer for further information.

Visit the ARL Statistics and Measurement Program online at http://www.arl.org/stats/.

Published by the

Association of Research Libraries

Washington, DC 20036

www.arl.org

ISSN 0361-5669

ISBN 1-59407-843-2

EAN 978-1-59407-843-9

(C) 2009

The compilation is copyrighted by the Association of Research Libraries. Blanket permission is granted to reproduce and distribute copies of this work for nonprofit, educational, or library purposes, provided that the author, source, and copyright notice are included on each copy. This permission is in addition to rights of reproduction granted under Sections 107, 108, and other provisions of the US Copyright Act.

The paper used in this publication meets the minimum requirements of the American National Standard for Information Science and National Information Standards Organization standard-Permanence of Paper for Publications and Documents in Libraries and Archives, ANSI/NISO Z39.48-1992(R1997). 


\section{CONTENTS}

Salary Survey Trends 2008-2009. 7

Figure 1: $\quad$ Ethnicity/Race of Professional Staff in US ARL University Libraries, 2008-2009 ................ 8

Figure 2: $\quad$ Minority Professionals by Region in US ARL University Libraries, 2008-2009.................... 9

Figure 3: $\quad$ Race/Ethnicity and Sex Distribution of Professional Staff in ARL University

Libraries, 2008-2009. .10

Figure 4: $\quad$ Distribution of Functional Specialist Job Sub-Codes by Type of Library ...............................12

Figure 5: Distribution of Functional Specialist Job Sub-Codes' Average Salary by Sex .......................12

\section{SAlary LeVels for Staff in ARL Libraries}

Table 1: $\quad$ Distribution by Salary Level 19

Table 2: $\quad$ Salary Trends in ARL University Libraries ............................................................................. 20

Table 3: $\quad$ Salary Trends in US ARL University Libraries..........................................................................21

Table 4: $\quad$ Salary Trends in Canadian ARL University Libraries ........................................................ 22

\section{ARL NONUNIVERSITY LIBRARIES}

Table 5: $\quad$ Median and Beginning Professional Salaries in ARL Nonuniversity Libraries 25

Table 6: $\quad$ Salary Trends in ARL Nonuniversity Libraries. .26

\section{ARL UnIVERsity LibRARIES}

Table 7: $\quad$ Filled Positions; Average, Median, Beginning Professional Salaries; and Average Years of Experience in ARL University Libraries, FY 2008-2009

Table 8: $\quad$ Beginning Professional Salaries in ARL University Libraries;

Rank Order Table, FY 2007-2008

Table 9: Beginning Professional Salaries in ARL University Libraries;

Rank Order Table, FY 2008-2009.

Table 10: $\quad$ Median Professional Salaries in ARL University Libraries;

Rank Order Table, FY 2007-2008 
Table 11: $\quad$ Median Professional Salaries in ARL University Libraries;

Table 12: $\quad$ Average Professional Salaries in ARL University Libraries;

Rank Order Table, FY 2007-2008 34

Table 13: $\quad$ Average Professional Salaries in ARL University Libraries;

Rank Order Table, FY 2008-2009. 35

Table 14: $\quad$ Average, Median, and Beginning Professional Salaries in ARL University

Libraries; Summary of Rankings, FY 2005-2006 to 2008-2009. 36

Table 15: $\quad$ Distribution of Professional Staff in ARL University Libraries

by Salary and Position, FY 2008-2009 40

Table 16: $\quad$ Distribution of Professional Staff in ARL University Libraries by Salary, Sex, and Position, FY 2008-2009

Table 17: $\quad$ Number and Average Salaries of ARL University Librarians

by Position and Sex, FY 2008-2009.

Table 18: $\quad$ Number and Average Years of Experience of ARL University Librarians

by Position and Sex, FY 2008-2009.

Table 19: $\quad$ Number and Average Salaries of ARL University Librarians

by Years of Experience and Sex, FY 2008-2009

Table 20: $\quad$ Average Salaries of ARL University Librarians

by Position and Years of Experience, FY 2008-2009

Table 21: $\quad$ Number and Average Salaries of ARL University Librarians

by Position and Type of Institution, FY 2008-2009

Table 22: $\quad$ Years of Experience of ARL University Librarians

by Position and Type of Institution, FY 2008-2009

Table 23: $\quad$ Number and Average Salaries of ARL University Librarians

by Position and Size of Professional Staff, FY 2008-2009.

Table 24: $\quad$ Years of Experience of ARL University Librarians

by Position and Size of Professional Staff, FY 2008-2009.

Table 25: $\quad$ Average Salaries of ARL University Librarians

by Position and Geographic Region, FY 2008-2009 50

ARL University Libraries by Geographic Region .51 


\section{US ARL UNIVERSITY LIBRARIES}

Table 26: $\quad$ Average Salaries of US ARL University Librarians by Position and Years of Experience, FY 2008-2009 54

Table 27: $\quad$ Number and Average Salaries of Minority US ARL University Librarians by Position and Sex, FY 2008-2009.

Table 28: $\quad$ Number and Average Years of Experience of Minority US ARL University

Librarians by Position and Sex, FY 2008-2009. 56

Table 29: $\quad$ Number and Average Salaries of US ARL University Librarians by Years of Experience and Sex, FY 2008-2009 .57

Table 30: $\quad$ Number and Average Salaries of Minority US ARL University Librarians by Years of Experience and Sex, FY 2008-2009.

\section{CANAdian ARL University Libraries}

Table 31: Filled Positions; Average, Median, and Beginning Professional Salaries; and Average Years of Professional Experience in Canadian ARL University Libraries, FY 2008-2009 . 60

Table 32: $\quad$ Number and Average Salaries of Canadian ARL University

Librarians by Position and Sex, FY 2008-2009

Table 33: $\quad$ Number and Average Years of Experience of Canadian ARL

University Librarians by Position and Sex, FY 2008-2009

Table 34: $\quad$ Number and Average Salaries of Canadian ARL University Librarians by Years of Experience and Sex, FY 2008-2009.

\section{ARL University Medical Libraries}

Table 35: $\quad$ Filled Positions; Average, Median, Beginning Professional Salaries; and Average Years of Professional Experience in ARL University Medical Libraries, FY 2008-2009 ..... 66

Table 36: Beginning Professional Salaries in ARL University Medical Libraries; Rank Order Table, FY 2008-2009.

Table 37: $\quad$ Median Professional Salaries in ARL University Medical Libraries;

Rank Order Table, FY 2008-2009

Table 38: $\quad$ Average Professional Salaries in ARL University Medical Libraries;

Rank Order Table, FY 2008-2009 
Table 39: $\quad$ Number and Average Salaries of ARL University Medical Librarians

by Position and Sex, FY 2008-2009. 71

Table 40: $\quad$ Number and Average Years of Experience of ARL University Medical Librarians by Position and Sex, FY 2008-2009. 72

Table 41: $\quad$ Number and Average Salaries of ARL University Medical Librarians by Years of Experience and Sex, FY 2008-2009. 73

\section{ARL University LAW LibRaries}

Table 42: $\quad$ Filled Positions; Average, Median, Beginning Professional Salaries; and Average Years of Experience in ARL University Law Libraries, FY 2008-2009. .76

Table 43: $\quad$ Beginning Professional Salaries in ARL University Law Libraries; Rank Order Table, FY 2008-2009 .78

Table 44: $\quad$ Median Professional Salaries in ARL University Law Libraries; Rank Order Table, FY 2008-2009 79

Table 45: $\quad$ Average Professional Salaries in ARL University Law Libraries; Rank Order Table, FY 2008-2009 80

Table 46: $\quad$ Number and Average Salaries of ARL University Law Librarians by Position and Sex, FY 2008-2009. .81

Table 47: $\quad$ Number and Average Years of Experience of ARL University Law Librarians by Position and Sex, FY 2008-2009. .82

Table 48: $\quad$ Number and Average Salaries of ARL University Law Librarians by Years of Experience and Sex, FY 2008-2009 83

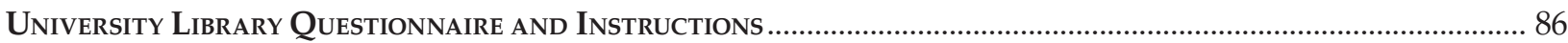

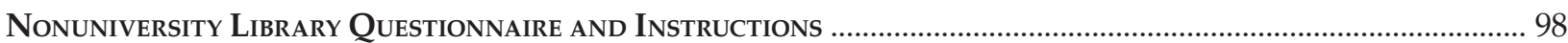

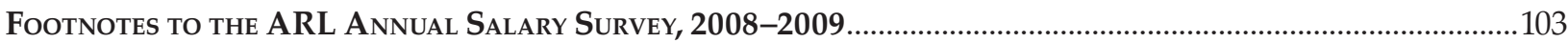

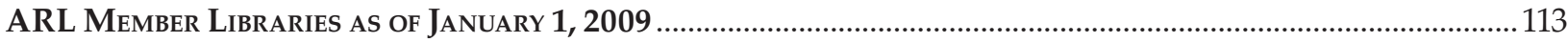

Table Numbering Changes for 1998-1999 to 1999-2000.... . .117 


\section{SAlary Survey Trends 2008-2009}

The ARL Annual Salary Survey 2008-2009 reports salary data for all professional staff working in ARL libraries. The Association of Research Libraries (ARL) represents the interests of libraries that serve major North American research institutions. The Association operates as a forum for the exchange of ideas and as an agent for collective action to influence forces affecting the ability of these libraries to meet the future needs of scholarship. The ARL Statistics and Measurement program, which produces the Salary Survey, is organized around collecting, analyzing, and distributing quantifiable information describing the characteristics of research libraries. The ARL Annual Salary Survey is the most comprehensive and thorough guide to current salaries in large US and Canadian academic and research libraries, and is a valuable management and research tool.

Data for 10,148 professional staff members were reported this year for the 113 ARL university libraries, including their law and medical libraries (941 staff members reported by 71 medical libraries and 743 staff members reported by 75 law libraries). For the 10 nonuniversity ARL members, data were reported for 3,748 professional staff members.

The tables are organized in seven major sections. The first section includes Tables 1 through 4, which report salary figures for all professionals working in ARL member libraries, including law and medical library data. The second section includes salary information for the 10 nonuniversity research libraries of ARL. The third section, entitled "ARL University Libraries," reports data in Tables 7 through 25 for the "general" library system of the university ARL members, combining US and Canadian data but excluding law and medical data. The fourth section, composed of Tables 26 through 30, reports data on US ARL university library members excluding law and medical data; the fifth section, Tables 31-34, reports data on Canadian ARL university libraries excluding law and medical data. The sixth section (Tables 35-41) and the seventh section (Tables 42-48) report on medical and law libraries, respectively, combining US and Canadian data.

The university population is generally treated in three distinct groups: staff in the "general" library system, staff in the university medical libraries, and staff in the university law libraries. Any branch libraries for which data were received, other than law and medical, are included in the "general" category, whether or not those libraries are administratively independent. Footnotes for many institutions provide information on branch inclusion or exclusion.

In all tables where data from US and Canadian institutions are combined, Canadian salaries are converted into US dollar equivalents at the rate of 1.0101 Canadian dollars per US dollar. Tables 4 and 31 through 34, however, pertain exclusively to staff in Canadian university libraries, so salary data in those tables are expressed in Canadian dollars.

\footnotetext{
${ }^{1}$ This is the average monthly noon exchange rate published in the Bank of Canada Review for the period July 2007-June 2008 and is used in converting figures that are shown effective as of 1 July 2008. This information can be accessed at: http://www.bankofcanada.ca/en/rates/ exchange.html. 


\section{RACE AND ETHNICITY}

There were 1,289 minority professional staff reported in 99 US ARL university libraries, including law and medical. ${ }^{2}$ Note that the data for minority professionals comes only from the US ARL university libraries following the Equal Employment Opportunity Commission (EEOC) definitions; Canadian law prohibits the identification of Canadians by ethnic category.

Currently, $14.1 \%$ of the professional staff in US ARL university libraries (including law and medical) belongs to one of the four non-Caucasian categories for which ARL keeps records. The percentage of minorities in managerial or leadership positions in the largest US academic libraries is far lower: $6.1 \%$ are directors (6 out of 97), 6.9\% are associate or assistant directors (29 out of 420), and $11.2 \%$ are branch librarians (50 out of 443 ). Figure 1, below, depicts the overall racial/ethnic distribution of professional staff in US ARL university libraries: Caucasian/Other 85.9\%, Asian/Pacific Islander 6.3\%, Black 4.7\%, Hispanic 2.8\%, and American Indian/Alaskan Native $0.3 \%$. According to a 1998 survey by Mary Jo Lynch, data from the American Library Association (ALA) show that the sample of academic libraries surveyed by ALA has a higher representation of Blacks, Asian/Pacific Islanders, and American Indian/Alaskan Native than ARL libraries. ${ }^{3}$

\section{Figure 1: Ethnicity/Race of Professional Staff in US ARL University Libraries, 2008-2009}

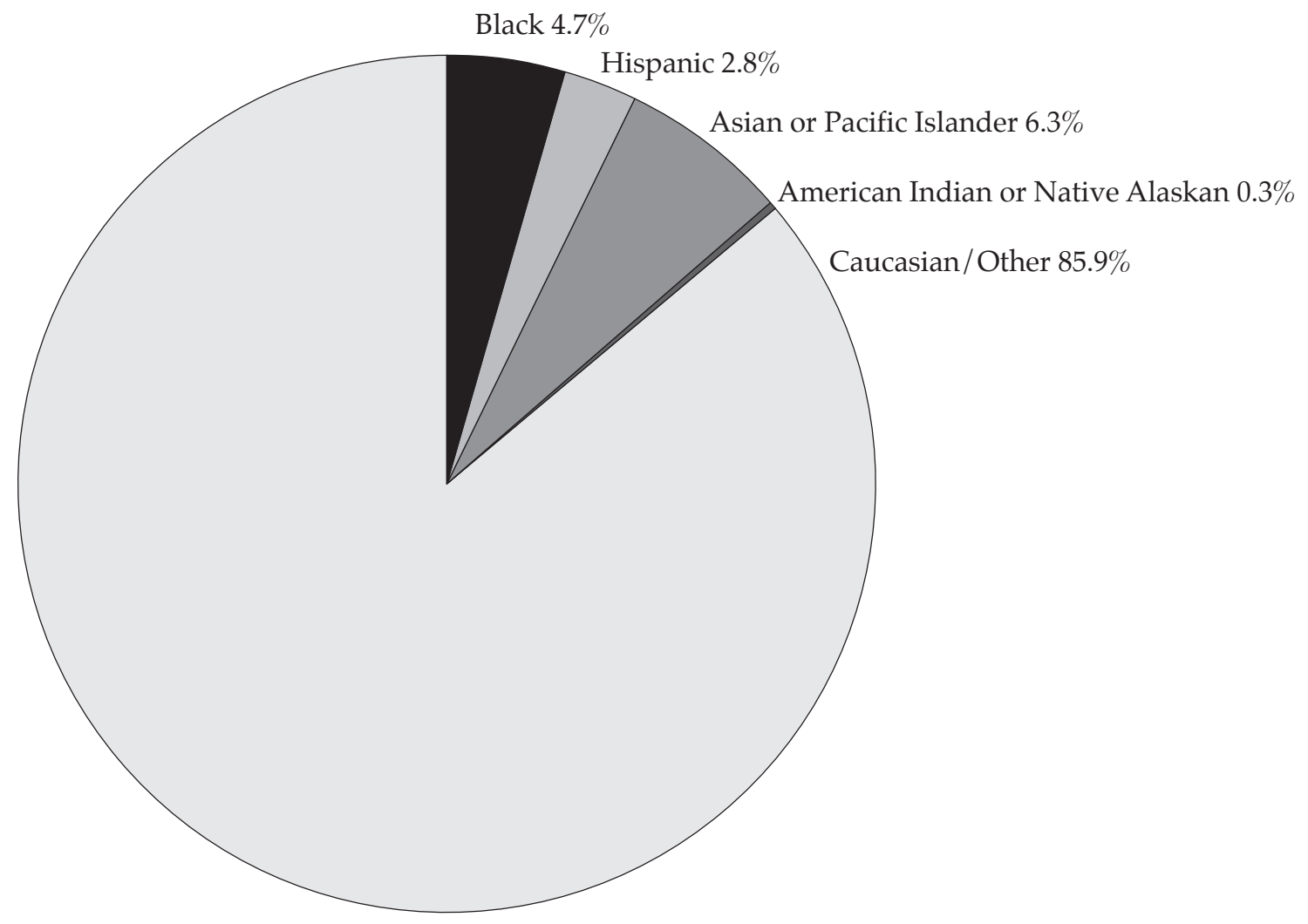

\footnotetext{
${ }^{2}$ Some US institutions offer their librarians the option of not reporting race and ethnicity; others forbid the tracking of racial and ethnic classification altogether. See Footnotes.

${ }^{3}$ Mary Jo Lynch, “Librarians' Salaries: Smaller Increases This Year," American Libraries 29, no. 10 (1998): 66-70. Also available at http://www. ala.org/alaorg/ors/racethnc.html.
}

8· ARL Annual Salary Survey 2008-2009 
Minority professional staff in US ARL university libraries continues to be disproportionately distributed across the country. Using Figure 2, we can compare the number of minority staff with other staff, region by region. These patterns of distribution have been relatively stable for the entire history of ARL's data-collection experience. Minorities are underrepresented over 36\% in the East South Central and by more than 25\% in the New England, West North Central and Mountain regions (see Table 25 for a definition of the regions). Proportionately to other regions, there are more minorities in the South Atlantic, West South Central, and Pacific regions.)

Figure 2: Minority Professionals by Region in US ARL University Libraries, 2008-2009

\begin{tabular}{|c|c|c|c|c|c|c|c|c|c|c|c|}
\hline & $\begin{array}{l}\text { New } \\
\text { England }\end{array}$ & $\begin{array}{l}\text { Middle } \\
\text { Atlantic }\end{array}$ & $\begin{array}{c}\text { East } \\
\text { North } \\
\text { Central }\end{array}$ & $\begin{array}{c}\text { West } \\
\text { North } \\
\text { Central }\end{array}$ & $\begin{array}{l}\text { South } \\
\text { Atlantic }\end{array}$ & $\begin{array}{c}\text { East } \\
\text { South } \\
\text { Central }\end{array}$ & $\begin{array}{c}\text { West } \\
\text { South } \\
\text { Central }\end{array}$ & Mountain & Pacific & TOTAL & $\%$ \\
\hline $\begin{array}{l}\text { Race/Ethnicity } \\
\text { Category }\end{array}$ & & & & & & & & & & & \\
\hline Black & 35 & 61 & 95 & 24 & 118 & 23 & 33 & 9 & 30 & 428 & $33 \%$ \\
\hline Hispanic & 26 & 41 & 22 & 11 & 44 & 4 & 39 & 25 & 44 & 256 & $20 \%$ \\
\hline Asian & 75 & 99 & 68 & 27 & 83 & 10 & 37 & 17 & 164 & 580 & $45 \%$ \\
\hline $\mathbf{A I} / \mathbf{A N}^{*}$ & 4 & 2 & 3 & 3 & 5 & 1 & 2 & 5 & & 25 & $2 \%$ \\
\hline Minority Total & 140 & 203 & 188 & 65 & 250 & 38 & 111 & 56 & 238 & 1,289 & $100 \%$ \\
\hline $\begin{array}{l}\text { Minority } \\
\text { Percent }\end{array}$ & $10.86 \%$ & $15.74 \%$ & $14.58 \%$ & $5.04 \%$ & $19.39 \%$ & $2.95 \%$ & $8.61 \%$ & $4.34 \%$ & $18.46 \%$ & & \\
\hline $\begin{array}{l}\text { Nonminority } \\
\text { Total }\end{array}$ & 1,171 & 1,250 & 1,398 & 535 & 1,256 & 366 & 583 & 464 & 846 & 7,869 & $100 \%$ \\
\hline $\begin{array}{l}\text { Nonminority } \\
\text { Percent }\end{array}$ & $14.88 \%$ & $15.88 \%$ & $17.76 \%$ & $6.79 \%$ & $15.96 \%$ & $4.65 \%$ & $7.40 \%$ & $5.89 \%$ & $10.75 \%$ & & \\
\hline $\begin{array}{l}\text { Regional } \\
\text { Percent } \\
\text { Total staff }\end{array}$ & $14.31 \%$ & $15.86 \%$ & $17.31 \%$ & $6.55 \%$ & $16.44 \%$ & $4.41 \%$ & $7.57 \%$ & $5.67 \%$ & $11.83 \%$ & & \\
\hline $\begin{array}{l}\text { Proportional } \\
\text { Minority } \\
\text { Representation }\end{array}$ & $-27.02 \%$ & $-0.88 \%$ & $-17.91 \%$ & $-25.77 \%$ & $21.49 \%$ & $-36.56 \%$ & $16.35 \%$ & $-26.32 \%$ & $71.72 \%$ & & \\
\hline
\end{tabular}

* American Indian/Alaskan Native

ARL recognizes the difficulties that the profession has in attracting a diverse workforce and continues to work actively in the development of workplace climates that embrace diversity. The ARL Diversity Program, through its Leadership and Career Development Program and the Initiative to Recruit a Diverse Workforce, 
emphasizes ARL and its members' commitment to creating a diverse academic and research library community to better meet the new challenges of global competition and changing demographics. Further, the Diversity Program focuses on issues surrounding work relationships in libraries while considering the impact of diversity on library services, interactions with library users, and the development of collections, at its homepage, http:// www.arl.org/diversity/.

Women comprise $69.1 \%$ of the four racial/ethnic groups that comprise minority staff, as compared to $62.8 \%$ of Caucasian/Other staff in all US ARL university libraries. The overall gender balance in the 113 Canadian and US university libraries (including law and medical) is 35.8\% male and 64.2\% female. See Figure 2, above, and Figure 3, below, for more detail on race/ethnic and gender distribution.

Figure 3: Race/Ethnicity and Sex Distribution of Professional Staff in ARL University Libraries, 2008-2009

\begin{tabular}{|c|c|c|c|c|c|}
\hline \multicolumn{6}{|c|}{ UNITED STATES } \\
\hline & \multicolumn{2}{|c|}{ Men } & \multicolumn{2}{|c|}{ Women } & \multirow[t]{2}{*}{ Total } \\
\hline & Number of Staff & Percent of Total & Number of Staff & Percent of Total & \\
\hline Main & 2,846 & $37.4 \%$ & 4,754 & $62.6 \%$ & 7,600 \\
\hline Medical & 243 & $28.5 \%$ & 610 & $71.5 \%$ & 853 \\
\hline Law & 237 & $33.6 \%$ & 468 & $66.4 \%$ & 705 \\
\hline Minority * & 398 & $30.9 \%$ & 891 & $69.1 \%$ & 1,289 \\
\hline Nonminority & 2,928 & $37.2 \%$ & 4,941 & $62.8 \%$ & 7,869 \\
\hline All & 3,326 & $36.3 \%$ & 5,832 & $63.7 \%$ & 9,158 \\
\hline \multicolumn{6}{|c|}{ Canada } \\
\hline & \multicolumn{2}{|c|}{ Men } & \multicolumn{2}{|c|}{ Women } & Total \\
\hline & Number of Staff & Percent of Total & Number of Staff & Percent of Total & \\
\hline Main & 281 & $32.5 \%$ & 583 & $67.5 \%$ & 864 \\
\hline Medical & 10 & $11.4 \%$ & 78 & $88.6 \%$ & 88 \\
\hline Law & 12 & $31.6 \%$ & 26 & $68.4 \%$ & 38 \\
\hline All & 303 & $30.6 \%$ & 687 & $69.4 \%$ & 990 \\
\hline \multicolumn{6}{|c|}{ United STATES AND CANADA (COMBINED) } \\
\hline & \multicolumn{2}{|c|}{ Men } & \multicolumn{2}{|c|}{ Women } & Total \\
\hline & Number of Staff & Percent of Total & Number of Staff & Percent of Total & \\
\hline Main & 3,127 & $36.9 \%$ & 5,337 & $63.1 \%$ & 8,464 \\
\hline Medical & 253 & $26.9 \%$ & 688 & $73.1 \%$ & 941 \\
\hline Law & 249 & $33.5 \%$ & 494 & $66.5 \%$ & 743 \\
\hline All & 3,629 & $35.8 \%$ & 6,519 & $64.2 \%$ & 10,148 \\
\hline
\end{tabular}

* Includes staff in medical and law libraries. 


\section{GENDER DATA}

Many readers of previous surveys have inquired about evidence of gender-based salary differentials in ARL libraries. Additionally, data on salary comparisons for directors also are frequently requested. In 2008-2009, in contrast to previous years, the average salary for female directors was higher than that of their male counterparts (see Table 17); furthermore, the number of women in the top administrative library position has continued to increase steadily in recent years (63 women directors out of 111 total directorships reported).

Looking at other job categories in Table 17 demonstrates this increase in women's salaries relative to men. In 14 of 27 job categories used in the table women's salaries were larger than men's. However, the salaries for women have not yet met parity with that of men: in 2008-2009 the overall salary for women was only $96.2 \%$ that of men for the 113 ARL university libraries (compared to $95.39 \%$ in 2007-2008). This suggests a slow, long-term trend towards closure of the gender gap in ARL libraries - in 1980-1981, women in ARL libraries made roughly $87 \%$ that of men.

Table 18 provides average years of professional experience for many of the same staffing categories for which salary data are shown in Table 17, revealing that experience differentials may explain some differences within specific job categories. Women have more experience in all but two of the 14 job categories in which they average higher pay, but there are other categories in which women, on average, have more experience and less pay: Assistant Director, Functional Specialist, and Department Head-Other. Table 19 further reveals that the average salary for men is consistently higher than the average salary for women in all ten of the experience cohorts, a pattern that is also repeated for minority librarians: the average salary for minority men is higher than that for minority women in nine out of the ten experience cohorts (see Table 30).

There is a sense that the gender gap persists in academe in areas beyond the library and that a renewed commitment to resolve the problem is needed. ${ }^{4} \mathrm{~A}$ variety of reasons have been offered as to why these trends persist, most notably the perception that work is peripheral in a woman's life and, consequently, femaledominated professions are undervalued. Librarianship is predominantly and persistently a woman's profession. The scarcity of men in the profession has been well documented in many studies — the largest percentage of men employed in ARL libraries was 38.2\% in 1980-1981; since then men have consistently represented about 35\% of the professional staff in ARL libraries.

\section{The Functional Specialist Breakdown}

In 2004, the ARL Statistics and Measurement Committee accepted a proposal from the ACRL Personnel Administrators and Staff Development Officers Discussion Group to break down the Functional Specialist category (FSPEC). The Group's major concern was that so many different types of positions, with their varying job descriptions and salaries, were being labeled with the code FSPEC that data reported for the category were beginning to lose meaning. For each position that would have been labeled FSPEC in past years, the proposal offered ARL institutions two options: either use one of eight new codes to describe that position; or, if none of the eight new codes could adequately describe that position, use FSPEC. As seen in Figure 4, only 17\% of Functional Specialists in all libraries did not use an alternative code, a slight decrease from 20.2\% in 2006-2007. Of the positions that did use an alternate code, $61.1 \%$ of them were Archivists or Information Technology specialists.

\footnotetext{
4 There are many instances citing the continuation of gender inequity in academia. See, for example: Denise K. Manger's articles in the Chronicle of Higher Education, "Faculty Salaries Increased 3.7\% in 1999-2000" (14 Apr. 2000: A20) and "Faculty Salaries are Up 3.6\%, Double the Rate of Inflation" (23 April 1999: A16); D. W. Miller, "Salary Gap Between Male and Female Professors Grows Over the Years, Study Suggests," Chronicle of Higher Education, Today's News, 27 April 2000, <http://chronicle.com/daily/2000/ 04/2000042702n.htm>; and Yolanda Moses, "Salaries in Academe: The Gender Gap Persists," Chronicle of Higher Education 12 December 1997: A60.
} 
Figure 4: Distribution of Functional Specialist Job Sub-Codes by Type of Library

\begin{tabular}{|l|c|c|c|c|c|c|c|c|}
\hline \multirow{2}{*}{ Position } & \multicolumn{2}{|c|}{ Main } & \multicolumn{2}{c|}{ Medical } & \multicolumn{2}{c|}{ Law } & \multicolumn{2}{c|}{ All } \\
\cline { 2 - 9 } & No. & Percent & No. & Percent & No. & Percent & No. & Percent \\
\cline { 1 - 9 } Archivist & 459 & $22.0 \%$ & 22 & $14.6 \%$ & 6 & $10.5 \%$ & 487 & $21.2 \%$ \\
Business Manager & 132 & $6.3 \%$ & 12 & $7.9 \%$ & 2 & $3.5 \%$ & 146 & $6.4 \%$ \\
Human Resources & 104 & $5.0 \%$ & 1 & $0.7 \%$ & 0 & $0.0 \%$ & 105 & $4.6 \%$ \\
IT - Programming & 408 & $19.5 \%$ & 32 & $21.2 \%$ & 15 & $26.3 \%$ & 455 & $19.8 \%$ \\
IT - Systems & 143 & $6.9 \%$ & 18 & $11.9 \%$ & 7 & $12.3 \%$ & 168 & $7.3 \%$ \\
IT - Web Development & 269 & $12.9 \%$ & 24 & $15.9 \%$ & 0 & $0.0 \%$ & 293 & $12.8 \%$ \\
Media/Multimedia & 110 & $5.3 \%$ & 6 & $4.0 \%$ & 4 & $7.0 \%$ & 120 & $5.2 \%$ \\
Preservation/Conservation & 127 & $6.1 \%$ & 1 & $0.7 \%$ & 3 & $5.3 \%$ & 131 & $5.7 \%$ \\
Other Functional Specialists & 335 & $16.0 \%$ & 35 & $23.1 \%$ & 20 & $35.1 \%$ & 390 & $17.0 \%$ \\
\hline Total & $\mathbf{2 , 0 8 7}$ & & $\mathbf{1 5 1}$ & & $\mathbf{5 7}$ & & $\mathbf{2 , 2 9 5}$ & \\
\hline
\end{tabular}

*Note: The 2007-2008 version of this table contained incorrect data and has been revised. For a revised edition go to http://www.arl.org/ stats/annualsurveys/salary/annualedssal.shtml.

Figure 5, below, displays the average salaries of the subcategories by position and sex in main libraries, in the same fashion as Table 17. The salaries in each of the sub-categories deviate widely from the combined Functional Specialist average salary of $\$ 63,928$. Human resource specialists have the highest average of all subcategories, with an average salary of $\$ 73,090$; media/multimedia specialists have the lowest average salary of $\$ 53,792$.

Figure 5: Distribution of Functional Specialist Job Sub-Codes' Average Salaries by Sex

\begin{tabular}{|c|c|c|c|c|c|c|}
\hline \multirow{2}{*}{ Position } & \multicolumn{2}{|c|}{ Women } & \multicolumn{2}{|c|}{ Men } & \multicolumn{2}{|c|}{ Total } \\
\hline & Salary & No. & Salary & No. & Salary & No. \\
\hline Archivist & $\$ 57,605$ & 290 & $\$ 61,830$ & 169 & $\$ 59,160$ & 459 \\
\hline Business Manager & 68,834 & 80 & 73,035 & 52 & 70,489 & 132 \\
\hline Human Resources & 72,464 & 86 & 76,080 & 18 & 73,090 & 104 \\
\hline IT - Programming & 66,700 & 141 & 65,009 & 267 & 65,593 & 408 \\
\hline IT - Systems & 62,047 & 67 & 63,819 & 76 & 62,989 & 143 \\
\hline IT - Web Development & 66,235 & 70 & 67,909 & 199 & 67,473 & 269 \\
\hline Media/Multimedia & 55,815 & 44 & 52,444 & 66 & 53,792 & 110 \\
\hline Preservation/Conservation & 60,664 & 93 & 65,900 & 34 & 62,066 & 127 \\
\hline Other Functional Specialists & 60,052 & 220 & 61,951 & 115 & 60,704 & 335 \\
\hline All Functional Specialists & $\$ 63,380$ & 1,091 & $\$ 65,331$ & 996 & $\$ 63,928$ & 2,087 \\
\hline
\end{tabular}

In regards to the gender gap in ARL libraries explained in the previous section, it is worth noting that the average salaries of men are higher than those of women in seven out of the nine categories in Figure 5. 


\section{Institutional Characteristics AND SAlaries}

\section{A. Public and Private Institutions}

The gap between salaries paid in private US ARL university libraries and those paid in publicly supported US university libraries increased slightly in 2008-2009 to 7\% (it was 6.2 in 2007-2008) with an average of $\$ 4,778$ more paid for a position in a private institution. Out of 27 job categories, only in four (Heads of Serials, Heads of Circulation, Heads of Rare Books/Manuscripts and Other department Heads) did librarians in public institutions earn more than their peers employed in private institutions (see Table 21).

\section{B. Library Size}

Library size, as measured by the number of professional staff, is another significant determinant of salary. As a rule, the largest libraries pay the highest average salaries, not only overall, but for specific positions as well. The cutoff staffing levels used to determine the largest cohort of libraries, after declining in every year since 19951996, continued to hold steady at 110 in 2008-2009.5 The largest libraries, those with more than 110 staff, reported the highest average salary, $\$ 73,135$, compared to $\$ 71,063$ for the libraries with between 75 and 110 staff (see Table 23). The smallest libraries (22-49 staff) had the third-highest average salary of the cohorts, followed by those libraries with 50 to 74 staff. One factor responsible for the high pay of the smallest libraries (22-49 staff) is related to the strengthening of the Canadian dollar relative to the US dollar (see Table 4 for a list of exchange rates since 1984-1985), as 11 of the 14 Canadian ARL members have less than 74 staff. The gap between the highest paying cohort and the lowest paying cohort dropped slightly, in 2008-2009 it was \$4,994, a difference of 6.83\% percent (the difference in $2007-08$ was $\$ 4,613$ or $7 \%$ ).

\section{Geographic Area}

In 2008-2009, the highest average salaries were found in Canada $(\$ 82,295)$ followed by the New England $(\$ 77,055)$ and Pacific $(\$ 74,056)$ regions (see Table 25$)$. This is partly a result of the US/Canadian exchange rate, which has dropped precipitously over the past five years (see Table 4). The West South Central region had the lowest average salary, with an average of $\$ 62,286$.

\section{RANK Structure}

Rank structure continues to provide a useful framework for examining professional salaries in ARL university libraries. Figure 6, below, displays average salary and years of experience in the most commonly used rank structures. Readers should be aware that not all individuals have a rank that fits into the rank structure the library utilizes. Most commonly, directors may have no rank (or a rank outside the structure) and it is common for non-librarians included in the survey (business officers, personnel staff, computer specialists, etc.) to be unranked, as well.

The pattern of relationships between rank and salary seen in past years continues: with higher rank associated with higher average years of experience and a correspondingly higher salary. 6,244 of the 8,463 librarians in ARL university member libraries occupy a rank within these three most commonly found ranking systems, and the largest number of professionals $(3,288)$ occupy a position in a four-step rank structure.

\footnotetext{
5 In 1995-1996, the largest cohort of libraries was determined based on staff over 124; in 1996-1998, over 120; in 1998-1999, over 115; and since 1999-2000, over 110. See Table 23.
} 
Figure 6: Average Salaries and Average Years of Experience of Library Professionals in Libraries with Three, Four, and Five Step Rank Structures, 2008-2009

\begin{tabular}{|c|c|c|c|c|c|c|}
\hline & \multicolumn{2}{|c|}{ Three-Step } & \multicolumn{2}{|c|}{ Four-Step } & \multicolumn{2}{|c|}{ Five-Step } \\
\hline & Salary & Experience & Salary & Experience & Salary & Experience \\
\hline Librarian 1 & 56,741 & 8.8 & 52,361 & 7.1 & 50,983 & 5.2 \\
\hline Librarian 2 & 67,919 & 17.5 & 56,097 & 10.4 & 58,104 & 10.8 \\
\hline Librarian 3 & 84,908 & 25.6 & 71,408 & 20.0 & 69,136 & 18.1 \\
\hline Librarian 4 & & & 86,622 & 25.8 & 86,903 & 23.9 \\
\hline Librarian 5 & & & & & 98,872 & 28 \\
\hline No. of Staff & \multicolumn{2}{|c|}{1,808} & \multicolumn{2}{|c|}{3,288} & \multicolumn{2}{|c|}{1,148} \\
\hline
\end{tabular}

\section{INFLATION EFFECT}

Tables 2 and 6 reveal changes in beginning professional and median salaries, as well as changes in the US Bureau of Labor's Cost of Living Index (CPI-All Urban Consumers) for university and nonuniversity research libraries. Table 3 is similar to Table 2, but reports data only on US libraries. Table 4 shows trend data for Canadian libraries and compares them to the Canadian Consumer Price Index changes. Tables 2, 3, and 4 include law and medical library staff in ARL university libraries. In contrast to 2007-08, these tables indicate that the purchasing power of professionals (in both the United States and Canada) employed in ARL libraries did not keep pace with inflation.

For a third consecutive year, the median Canadian salary (converted to US dollars) exceeded the median US salary. The median salary for all ARL university libraries was $\$ 64,828$ in 2008-2009; for Canadian libraries converted into US dollars it was $\$ 77,954$ (a median of $\$ 78,742$ in Canadian dollars); and for US libraries it was only $\$ 63,673$. However, in 2008-2009 US median salaries increased 3.8\% (see Table 3), in comparison, Canadian median salaries (when denominated in Canadian dollars and compared against the Canadian CPI) increased only 3.3\% (see Table 4). The median salary for combined US and Canadian university libraries increased $4.8 \%$ (Table 2); at the same time, the US Consumer Price Index increased 5.6\% (see Table 3) in the last year and the Canadian Consumer Price Index increased 3.4\% (see Table 4).

Beginning salaries in the university sector continue to increase at a steady rate. The median beginning salary in ARL university libraries rose to $\$ 44,000$; this is a $6.6 \%$ increase over the median beginning salary of $\$ 41,125$ reported in 2007-2008 (see Table 2). The median beginning salary for ARL nonuniversity research libraries also expanded from $\$ 44,359$ in 2007-08 to $\$ 48,108$; an increase 7.8\% (see Table 6). Table 6 also reveals that the median salary for nonuniversity staff has increased about 6\% in 2008-2009 to \$85,320.

Libraries need staff with high-level technical skills to operate at the more sophisticated and complex information environments that are in place. As people are hired with higher beginning salaries, the inability to adjust the overall salary structure to achieve some equity for the experienced staff members is another factor that contributes to slow salary growth for higher salaries. 
Readers are reminded that these data reflect only salaries, and that there are other compensation issues which may have influenced the pattern of salaries in various institutions. In addition, a highly standardized structure for capturing data has been used, which may portray results in a way that cannot be fully representative of a local situation.

Martha Kyrillidou

Les Bland

Association of Research Libraries

April 1, 2009 

Salary LeVels for Staff in ARL Libraries

TABles 1-4 



\section{Table 1: Distribution by Salary LeVel}

Figures in columns headed by fiscal year show the number of filled professional positions. Columns headed by Cum. \% show the percentage of all filled positions with salaries equal to or more than the beginning of each salary range. For example: in FY 2008-2009, 82.8\% of all ARL university librarians earned more than $\$ 50,000$, as did $96 \%$ of all ARL nonuniversity librarians.

Note: Canadian salaries are expressed in US dollars. Data includes medical and law libraries.

\begin{tabular}{|c|c|c|c|c|c|c|c|c|}
\hline \multirow[b]{3}{*}{ Salary Range } & \multicolumn{4}{|c|}{ University Librarians } & \multicolumn{4}{|c|}{ Nonuniversity Librarians } \\
\hline & FY & Cum. & FY & Cum. & FY & Cum. & FY & Cum. \\
\hline & 2007-2008 & $\%$ & 2008-2009 & $\%$ & $2007-2008$ & $\%$ & 2008-2009 & $\%$ \\
\hline More than 250,000 & 12 & $0.1 \%$ & 17 & $0.2 \%$ & 1 & $0.0 \%$ & 1 & $0.0 \%$ \\
\hline $200,000-250,000$ & 30 & $0.4 \%$ & 36 & $0.5 \%$ & 1 & $0.1 \%$ & 2 & $0.1 \%$ \\
\hline $175,000-199,999$ & 41 & $0.8 \%$ & 55 & $1.1 \%$ & 7 & $0.2 \%$ & 3 & $0.2 \%$ \\
\hline $150,000-174,999$ & 81 & $1.6 \%$ & 91 & $2.0 \%$ & 108 & $3.1 \%$ & 112 & $3.1 \%$ \\
\hline $140,000-149,999$ & 37 & $2.0 \%$ & 55 & $2.5 \%$ & 148 & $7.0 \%$ & 197 & $8.4 \%$ \\
\hline $130,000-139,999$ & 57 & $2.6 \%$ & 87 & $3.4 \%$ & 118 & $10.1 \%$ & 115 & $11.5 \%$ \\
\hline $120,000-129,999$ & 90 & $3.55 \%$ & 124 & $4.6 \%$ & 223 & $16.0 \%$ & 251 & $18.2 \%$ \\
\hline $110,000-119,999$ & 177 & $5.3 \%$ & 229 & $6.8 \%$ & 199 & $21.2 \%$ & 237 & $24.5 \%$ \\
\hline $100,000-109,999$ & 246 & $7.7 \%$ & 369 & $10.5 \%$ & 351 & $30.4 \%$ & 373 & $34.4 \%$ \\
\hline 95,000 - 99,999 & 212 & $9.8 \%$ & 296 & $13.4 \%$ & 219 & $36.2 \%$ & 241 & $40.9 \%$ \\
\hline 90,000 - 94,999 & 299 & $12.8 \%$ & 296 & $16.3 \%$ & 142 & $40.0 \%$ & 312 & $49.2 \%$ \\
\hline $85,000-89,999$ & 374 & $16.6 \%$ & 413 & $20.4 \%$ & 295 & $47.7 \%$ & 195 & $54.4 \%$ \\
\hline $80,000-84,999$ & 469 & $21.3 \%$ & 568 & $26.0 \%$ & 285 & $55.2 \%$ & 244 & $60.9 \%$ \\
\hline $76,000-79,999$ & 456 & $25.9 \%$ & 479 & $30.7 \%$ & 146 & $59.1 \%$ & 179 & $65.7 \%$ \\
\hline $74,000-75,999$ & 229 & $28.1 \%$ & 322 & $33.9 \%$ & 88 & $61.4 \%$ & 130 & $69.2 \%$ \\
\hline $72,000-73,999$ & 330 & $31.5 \%$ & 299 & $36.8 \%$ & 180 & $66.1 \%$ & 88 & $71.5 \%$ \\
\hline 70,000 - 71,999 & 306 & $34.5 \%$ & 301 & $39.8 \%$ & 119 & $69.3 \%$ & 92 & $74.0 \%$ \\
\hline $68,000-69,999$ & 312 & $37.6 \%$ & 410 & $43.8 \%$ & 120 & $72.4 \%$ & 114 & $77.0 \%$ \\
\hline $66,000-67,999$ & 408 & $41.7 \%$ & 351 & $47.3 \%$ & 87 & $74.7 \%$ & 40 & $78.1 \%$ \\
\hline $64,000-65,999$ & 363 & $45.4 \%$ & 473 & $51.9 \%$ & 95 & $77.2 \%$ & 123 & $81.4 \%$ \\
\hline $62,000-63,999$ & 436 & $49.7 \%$ & 365 & $55.5 \%$ & 75 & $79.2 \%$ & 83 & $83.6 \%$ \\
\hline $60,000-61,999$ & 397 & $53.7 \%$ & 523 & $60.7 \%$ & 80 & $81.3 \%$ & 114 & $86.6 \%$ \\
\hline $58,000-59,999$ & 494 & $58.7 \%$ & 420 & $64.8 \%$ & 138 & $84.9 \%$ & 159 & $90.8 \%$ \\
\hline $56,000-57,999$ & 472 & $63.4 \%$ & 466 & $69.4 \%$ & 78 & $87.0 \%$ & 46 & $92.1 \%$ \\
\hline $54,000-55,999$ & 511 & $68.5 \%$ & 441 & $73.8 \%$ & 135 & $90.5 \%$ & 34 & $93.0 \%$ \\
\hline $52,000-53,999$ & 510 & $73.6 \%$ & 475 & $78.4 \%$ & 104 & $93.3 \%$ & 65 & $94.7 \%$ \\
\hline $50,000-51,999$ & 500 & $78.6 \%$ & 441 & $82.8 \%$ & 52 & $94.7 \%$ & 47 & $96.0 \%$ \\
\hline $48,000-49,999$ & 459 & $83.2 \%$ & 417 & $86.9 \%$ & 46 & $95.9 \%$ & 66 & $97.7 \%$ \\
\hline 46,000 - 47,999 & 440 & $87.6 \%$ & 361 & $90.5 \%$ & 98 & $98.4 \%$ & 30 & $98.5 \%$ \\
\hline $44,000-45,999$ & 384 & $91.5 \%$ & 321 & $93.6 \%$ & 15 & $98.8 \%$ & 7 & $98.7 \%$ \\
\hline $42,000-43,999$ & 321 & $94.7 \%$ & 217 & $95.8 \%$ & 12 & $99.2 \%$ & 24 & $99.4 \%$ \\
\hline $40,000-41,999$ & 217 & $96.9 \%$ & 182 & $97.6 \%$ & 8 & $99.4 \%$ & 3 & $99.4 \%$ \\
\hline $38,000-39,999$ & 132 & $98.2 \%$ & 109 & $98.6 \%$ & 4 & $99.5 \%$ & 9 & $99.7 \%$ \\
\hline $36,000-37,999$ & 92 & $99.1 \%$ & 60 & $99.2 \%$ & 10 & $99.7 \%$ & 0 & $99.7 \%$ \\
\hline $34,000-35,999$ & 41 & $99.5 \%$ & 43 & $99.6 \%$ & 1 & $99.8 \%$ & 0 & $99.7 \%$ \\
\hline $32,000-33,999$ & 24 & $99.8 \%$ & 21 & $99.9 \%$ & 8 & $100.0 \%$ & 0 & $99.7 \%$ \\
\hline $30,000-31,999$ & 14 & $99.9 \%$ & 8 & $99.9 \%$ & 0 & $100.0 \%$ & 11 & $100.0 \%$ \\
\hline less than 30,000 & 10 & $100.0 \%$ & 7 & $100.0 \%$ & 1 & $100.0 \%$ & 1 & $100.0 \%$ \\
\hline Total Positions & & 9,983 & & 10,148 & & 3,797 & & 3,748 \\
\hline Median Salary & & $\$ 61,833$ & & $\$ 64,828$ & & $\$ 80,261$ & & $\$ 85,320$ \\
\hline
\end{tabular}




\section{Table 2: Salary Trends in ARL University Libraries}

Salary figures for the current year are displayed in the context of the previous years and compared to the changes in the Consumer Price Index (CPI) to show trends in the purchasing power of median and beginning professional salaries. Salary figures and CPI numbers have been converted to adjusted indexes using July 1984 as the base. Actual CPI data retrieved from the US Department of Labor, Bureau of Labor Statistics' Consumer Price Index-All Urban Consumers (US All items, 1982-1984=100 - CUUR0000SA0) available online at http://www.bls. gov/data/.

Note: Canadian salaries are expressed in US dollars.

\begin{tabular}{|c|c|c|c|c|c|c|c|c|}
\hline $\begin{array}{l}\text { Fiscal } \\
\text { Year }\end{array}$ & $\begin{array}{l}\text { Number of } \\
\text { Libraries }\end{array}$ & $\begin{array}{l}\text { Total } \\
\text { Staff }\end{array}$ & $\begin{array}{c}\text { Median } \\
\text { Salary }^{+}\end{array}$ & $\begin{array}{c}\text { BPS }^{\ddagger} \\
\text { Median }\end{array}$ & $\begin{array}{c}\text { Median } \\
\text { Salary Index }\end{array}$ & $\begin{array}{l}\text { BPS }^{\ddagger} \\
\text { Index }\end{array}$ & $\begin{array}{c}\text { Actual } \\
\text { CPI }^{*}\end{array}$ & $\begin{array}{c}\text { Adjusted } \\
\text { CPI }\end{array}$ \\
\hline 2008-2009 & 113 & 10,148 & $\$ 64,823$ & $\$ 44,000$ & 248.4 & 266.7 & 219.9 & 211.6 \\
\hline 2007-2008 & 113 & 9,983 & 61,833 & 41,125 & 236.9 & 249.7 & 208.3 & 200.5 \\
\hline 2006-2007 & 113 & 9,824 & 59,648 & 40,000 & 228.5 & 242.4 & 203.5 & 195.9 \\
\hline 2005-2006 & 113 & 9,655 & 57,074 & 37,920 & 218.7 & 229.8 & 195.4 & 188.1 \\
\hline 2004-2005 & 113 & 9,487 & 55,250 & 36,984 & 211.7 & 224.1 & 189.4 & 182.3 \\
\hline 2003-2004 & 114 & 9,492 & 53,000 & 36,000 & 203.1 & 218.2 & 183.9 & 177.0 \\
\hline 2002-2003 & 114 & 9,469 & 51,636 & 35,000 & 197.8 & 212.1 & 180.1 & 173.3 \\
\hline 2001-2002 & 113 & 9,198 & 50,724 & 34,000 & 194.3 & 206.1 & 177.5 & 170.8 \\
\hline $2000-2001$ & 112 & 8,882 & 49,068 & 32,879 & 188.0 & 199.3 & 172.8 & 166.3 \\
\hline $1999-2000$ & 111 & 8,595 & 47,377 & 31,100 & 181.5 & 188.5 & 166.7 & 160.4 \\
\hline 1998-1999 & 110 & 8,400 & 45,775 & 30,000 & 175.2 & 181.7 & 163.2 & 157.1 \\
\hline 1997-1998 & 110 & 8,414 & 44,534 & 28,500 & 170.5 & 172.6 & 160.5 & 154.5 \\
\hline 1996-1997 & 109 & 8,325 & 43,170 & 27,687 & 165.3 & 167.7 & 157.0 & 151.1 \\
\hline 1995-1996 & 108 & 8,231 & 41,901 & 27,000 & 160.5 & 163.6 & 152.5 & 146.8 \\
\hline 1994-1995 & 108 & 8,216 & 41,088 & 26,000 & 157.4 & 157.6 & 148.4 & 142.8 \\
\hline 1993-1994 & 108 & 8,132 & 40,225 & 25,834 & 154.1 & 156.6 & 144.4 & 139.0 \\
\hline 1992-1993 & 108 & 8,212 & 39,265 & 25,000 & 150.4 & 151.5 & 140.5 & 134.9 \\
\hline 1991-1992 & 107 & 8,256 & 38,537 & 24,000 & 147.7 & 145.5 & 136.2 & 131.1 \\
\hline 1990-1991 & 107 & 8,382 & 36,701 & 23,800 & 140.6 & 144.2 & 130.4 & 125.8 \\
\hline 1989-1990 & 107 & 8,253 & 34,629 & 22,000 & 132.7 & 133.3 & 124.4 & 119.3 \\
\hline 1988-1989 & 107 & 8,087 & 32,461 & 20,400 & 124.4 & 123.6 & 118.5 & 113.9 \\
\hline 1987-1988 & 106 & 7,962 & 30,534 & 19,460 & 117.0 & 117.9 & 113.8 & 109.3 \\
\hline 1986-1987 & 105 & 7,718 & 28,941 & 18,250 & 110.9 & 110.6 & 109.5 & 105.5 \\
\hline 1985-1986 & 105 & 7,543 & 27,485 & 17,500 & 105.3 & 106.1 & 107.8 & 103.6 \\
\hline 1984-1985 & 104 & 7,161 & 26,100 & 16,500 & 100.0 & 100.0 & 104.1 & 100.0 \\
\hline
\end{tabular}




\section{TAble 3: Salary Trends in US ARL University Libraries}

Salary figures for the current year are displayed in the context of previous years and compared to the changes in the US Consumer Price Index (CPI) to show trends in the purchasing power of median and beginning professional salaries. Salary figures and CPI numbers have been converted to adjusted indexes, using July 1984 as the base. Actual CPI data retrieved from the US Department of Labor, Bureau of Labor Statistics' Consumer Price Index-All Urban Consumers (US All items, 1982-1984=100 - CUUR0000SA0) available online at http://www. bls.gov/data/.

Note: Data includes medical and law libraries in median salary.

\begin{tabular}{|c|c|c|c|c|c|c|c|c|}
\hline $\begin{array}{l}\text { Fiscal } \\
\text { Year }\end{array}$ & $\begin{array}{l}\text { Number of } \\
\text { Libraries }\end{array}$ & $\begin{array}{l}\text { Total } \\
\text { Staff }\end{array}$ & $\begin{array}{c}\text { Median } \\
\text { Salary }^{+}\end{array}$ & $\begin{array}{c}\text { Median } \\
\text { Salary Change }\end{array}$ & $\begin{array}{c}\text { Median } \\
\text { Salary Index }\end{array}$ & $\begin{array}{l}\text { Actual } \\
\text { CPI }^{*}\end{array}$ & $\begin{array}{l}\text { Adjusted } \\
\text { CPI }\end{array}$ & $\begin{array}{c}\text { CPI } \\
\text { Change }\end{array}$ \\
\hline 2008-2009 & 99 & 9,158 & $\$ 63,673$ & $3.8 \%$ & 245.4 & 219.9 & 211.6 & $5.6 \%$ \\
\hline 2007-2008 & 99 & 9,026 & 61,329 & 3.5 & 236.4 & 208.3 & 200.5 & 2.4 \\
\hline 2006-2007 & 99 & 8,866 & 59,280 & 3.7 & 228.5 & 203.5 & 195.9 & 4.1 \\
\hline 2005-2006 & 99 & 8,700 & 57,173 & 2.8 & 220.4 & 195.4 & 188.1 & 3.2 \\
\hline 2004-2005 & 99 & 8,581 & 55,600 & 3.2 & 214.3 & 189.4 & 182.3 & 3.0 \\
\hline 2003-2004 & 100 & 8,581 & 53,859 & 2.0 & 207.6 & 183.9 & 177.0 & 2.1 \\
\hline 2002-2003 & 100 & 8,544 & 52,789 & 1.9 & 203.5 & 180.1 & 173.3 & 1.5 \\
\hline 2001-2002 & 99 & 8,337 & 51,806 & 4.1 & 199.7 & 177.5 & 170.8 & 2.7 \\
\hline 2000-2001 & 99 & 8,127 & 49,753 & 3.7 & 191.8 & 172.8 & 166.3 & 3.7 \\
\hline $1999-2000$ & 98 & 7,858 & 48,000 & 4.1 & 185.0 & 166.7 & 160.4 & 2.1 \\
\hline 1998-1999 & 97 & 7,671 & 46,130 & 3.6 & 177.8 & 163.2 & 157.1 & 1.7 \\
\hline 1997-1998 & 97 & 7,682 & 44,544 & 3.4 & 171.7 & 160.5 & 154.5 & 2.2 \\
\hline 1996-1997 & 96 & 7,562 & 43,084 & 3.4 & 166.1 & 157.0 & 151.1 & 3.0 \\
\hline 1995-1996 & 95 & 7,435 & 41,651 & 2.7 & 160.5 & 152.5 & 146.8 & 2.8 \\
\hline 1994-1995 & 95 & 7,401 & 40,573 & 3.4 & 156.4 & 148.4 & 142.8 & 2.8 \\
\hline 1993-1994 & 95 & 7,390 & 39,257 & 3.0 & 151.3 & 144.4 & 139.0 & 2.8 \\
\hline 1992-1993 & 95 & 7,375 & 38,124 & 3.0 & 146.9 & 140.5 & 134.9 & 3.2 \\
\hline 1991-1992 & 94 & 7,408 & 37,009 & 3.5 & 142.6 & 136.2 & 131.1 & 4.4 \\
\hline 1990-1991 & 94 & 7,543 & 35,761 & 5.2 & 137.8 & 130.4 & 125.8 & 4.8 \\
\hline 1989-1990 & 94 & 7,344 & 34,000 & 5.8 & 131.0 & 124.4 & 119.3 & 5.0 \\
\hline 1988-1989 & 94 & 7,252 & 32,149 & 5.4 & 123.9 & 118.5 & 113.9 & 4.1 \\
\hline 1987-1988 & 93 & 7,145 & 30,492 & 5.1 & 117.5 & 113.8 & 109.3 & 3.9 \\
\hline 1986-1987 & 92 & 6,886 & 29,021 & 6.5 & 111.9 & 109.5 & 105.5 & 1.6 \\
\hline 1985-1986 & 91 & 6,707 & 27,249 & 5.0 & 105.0 & 107.8 & 103.6 & 3.6 \\
\hline 1984-1985 & 91 & 6,456 & 25,946 & 6.9 & 100.0 & 104.1 & 100.0 & - \\
\hline
\end{tabular}

*Actual CPI and CPI figures have been revised from previous editions based upon changes published by the Bureau of Labor Statistics. These changes were caused by rounding; they are minute and are less than 0.3 in all cases.

† Includes medical and law libraries. 


\section{Table 4: Salary Trends in Canadian ARL University Libraries}

Salary figures for the current year are displayed in the context of previous years. Canadian salaries are presented in both US \$ and Canadian \$ denominations and the annual exchange rate used in the salary surveys is also listed. Canadian salaries are also compared to the changes in the Canadian Consumer Price Index (CPI) to show trends in the purchasing power of median Canadian salaries. CPI number changes are based on July CPI figures. The source for Canadian CPI data is Table 5: The Consumer Price Index for Canada, published in The Daily, a Statistics Canada publication, available online at http://www.statcan.gc.ca/pub/62-001-x/2008012/t040-eng.htm.

\begin{tabular}{|c|c|c|c|c|c|c|c|c|c|}
\hline $\begin{array}{l}\text { Fiscal } \\
\text { Year }\end{array}$ & $\begin{array}{l}\text { No. of } \\
\text { Libs. }\end{array}$ & $\begin{array}{l}\text { Total } \\
\text { Staff }\end{array}$ & $\begin{array}{l}\text { Median } \\
\text { Salary } \\
\text { in US } \$^{+}\end{array}$ & $\begin{array}{c}\text { Median } \\
\text { Salary } \\
\text { Change }^{+}\end{array}$ & $\begin{array}{c}\text { Exchange } \\
\text { Rate }\end{array}$ & $\begin{array}{l}\text { Median } \\
\text { Salary } \\
\text { in Can. \$ }\end{array}$ & $\begin{array}{c}\text { Median } \\
\text { Salary } \\
\text { Change }\end{array}$ & $\begin{array}{l}\text { Can. } \\
\text { CPI }^{* *}\end{array}$ & $\begin{array}{c}\text { Can. } \\
\text { CPI } \\
\text { Change* }\end{array}$ \\
\hline 2008-2009 & 14 & 990 & $\$ 77,954$ & $15.8 \%$ & 1.0101 & $\$ 78,742$ & $3.3 \%$ & 115.8 & $3.4 \%$ \\
\hline 2007-2008 & 14 & 957 & 67,331 & 6.7 & 1.1323 & 76,239 & 3.9 & 112.0 & 2.2 \\
\hline 2006-2007 & 14 & 958 & 63,112 & 11.8 & 1.16289 & 73,392 & 4.0 & 109.6 & 2.3 \\
\hline 2005-2006 & 14 & 955 & 56,474 & 7.1 & 1.24971 & 70,576 & -0.3 & 107.1 & 2.0 \\
\hline 2004-2005 & 14 & 906 & 52,707 & 16.3 & 1.34328 & 70,800 & 3.5 & 105.0 & 2.3 \\
\hline 2003-2004 & 14 & 911 & 45,310 & 6.2 & 1.51023 & 68,429 & 2.3 & 102.6 & 2.1 \\
\hline 2002-2003 & 14 & 925 & 42,657 & -0.6 & 1.56878 & 66,919 & 2.6 & 100.5 & 2.1 \\
\hline 2001-2002 & 14 & 861 & 42,928 & -1.1 & 1.51919 & 65,215 & 2.1 & 98.4 & 2.7 \\
\hline 2000-2001 & 13 & 755 & 43,394 & 5.0 & 1.47192 & 63,873 & 2.4 & 95.8 & 2.9 \\
\hline $1999-2000$ & 13 & 737 & 41,316 & -3.8 & 1.5103 & 62,400 & 2.4 & 93.1 & 1.9 \\
\hline 1998-1999 & 13 & 729 & 42,963 & -2.7 & 1.4177 & 60,909 & 0.9 & 91.4 & 1.0 \\
\hline 1997-1998 & 13 & 732 & 44,167 & 1.4 & 1.3663 & 60,346 & 1.7 & 90.5 & 1.7 \\
\hline 1996-1997 & 13 & 764 & 43,569 & 0.9 & 1.3613 & 59,310 & -0.4 & 89.0 & 1.3 \\
\hline 1995-1996 & 13 & 796 & 43,173 & -1.7 & 1.3794 & 59,554 & 1.3 & 87.9 & 2.6 \\
\hline 1994-1995 & 13 & 815 & 43,919 & -6.0 & 1.3381 & 58,768 & 0.7 & 85.7 & 0.1 \\
\hline 1993-1994 & 13 & 816 & 46,744 & -4.3 & 1.2488 & 58,374 & 2.9 & 85.6 & 1.7 \\
\hline 1992-1993 & 13 & 837 & 48,820 & 2.7 & 1.1623 & 56,744 & 3.4 & 84.2 & 1.2 \\
\hline 1991-1992 & 13 & 847 & 47,519 & 5.5 & 1.1547 & 54,870 & 3.6 & 83.2 & 6.0 \\
\hline 1990-1991 & 13 & 839 & 45,023 & 15.1 & 1.1759 & 52,942 & 12.5 & 78.5 & 4.1 \\
\hline 1989-1990 & 13 & 853 & 39,117 & 12.3 & 1.2026 & 47,042 & 5.3 & 75.4 & 5.3 \\
\hline 1988-1989 & 13 & 837 & 34,826 & 11.7 & 1.2826 & 44,668 & 5.3 & 71.6 & 3.9 \\
\hline 1987-1988 & 13 & 817 & 31,178 & 10.9 & 1.3602 & 42,408 & 9.1 & 68.9 & 4.6 \\
\hline 1986-1987 & 13 & 831 & 28,123 & -1.9 & 1.3817 & 38,858 & 1.2 & 65.9 & 4.1 \\
\hline 1985-1986 & 13 & 829 & 28,666 & 1.1 & 1.3388 & 38,378 & 7.9 & 63.3 & 4.1 \\
\hline 1984-1985 & 12 & 705 & 28,346 & -0.8 & 1.2548 & 35,569 & 0.8 & 60.8 & 4.1 \\
\hline Average & & & & $3.8 \%$ & & & $3.3 \%$ & & \\
\hline
\end{tabular}

${ }^{\dagger}$ Includes medical and law libraries in median salary.

* Canadian CPI change figures have been revised from previous editions based upon changes published by The Daily (Statistics Canada). These changes were caused by rounding; they are minute and are less than 0.3 in all cases.

** Actual Canadian CPI figures have been added to this table (not available in previous editions). 


\section{ARL NoNUNIVERSITY LIBRARIES}

TABles 5-6 

Table 5: Median and Beginning Professional Salaries in ARL Nonuniversity Libraries

\begin{tabular}{|c|c|c|c|c|c|}
\hline & \multirow{2}{*}{$\begin{array}{l}\text { No. of } \\
\text { Staff }\end{array}$} & \multicolumn{2}{|c|}{ Median Salaries* } & \multicolumn{2}{|c|}{ Beginning Salaries* } \\
\hline & & FY 2007-2008 & FY 2008-2009 & FY 2007-2008 & FY 2008-2009 \\
\hline Boston Public Library & 179 & $\$ 58,099$ & $\$ 63,736$ & $\$ 37,907$ & $\$ 39,782$ \\
\hline $\begin{array}{l}\text { Canada Institute for Scientific } \\
\text { and Technical Information *+ }\end{array}$ & 194 & 61,365 & 72,217 & 44,105 & 49,441 \\
\hline Center for Research Libraries ${ }^{+}$ & 29 & 51,000 & 42,348 & 44,584 & 33,878 \\
\hline Library of Congress $^{+}$ & 2,583 & 89,985 & 96,791 & 46,041 & 48,108 \\
\hline National Agricultural Library ${ }^{+}$ & 99 & 75,671 & 79,060 & 46,041 & 48,108 \\
\hline Library \& Archives of Canada * & 113 & 55,337 & 62,031 & 44,133 & 49,472 \\
\hline National Library of Medicine ${ }^{+}$ & 183 & 80,123 & 86,046 & 46,041 & 48,108 \\
\hline New York Public Library & 251 & 58,674 & 59,963 & 39,295 & 39,295 \\
\hline New York State Library & 59 & 58,849 & 58,849 & 42,693 & 47,556 \\
\hline Smithsonian Library & 58 & 75,671 & 81,394 & 46,041 & 49,712 \\
\hline
\end{tabular}

* Canadian salaries are expressed in US dollars.

+ See footnotes. 


\section{Table 6: Salary Trends in ARL Nonuniversity Libraries}

Salary figures for the current year are displayed in the context of the previous years and compared to the changes in the Consumer Price Index (CPI) to show trends in the purchasing power of median and beginning professional salaries. Salary figures and CPI numbers have been converted to adjusted indexes, using July 1984 as the base. Actual CPI data retrieved from the US Department of Labor, Bureau of Labor Statistics' Consumer Price Index-All Urban Consumers (US All items, 1982-1984=100 - CUUR0000SA0) available online at http://www. bls.gov/data/.

\begin{tabular}{|c|c|c|c|c|c|c|c|c|}
\hline $\begin{array}{l}\text { Fiscal } \\
\text { Year }\end{array}$ & $\begin{array}{l}\text { Number of } \\
\text { Libraries }\end{array}$ & $\begin{array}{l}\text { Total } \\
\text { Staff }\end{array}$ & $\begin{array}{l}\text { Median } \\
\text { Salary* }\end{array}$ & $\begin{array}{c}\text { BPS }^{+} \\
\text {Median }\end{array}$ & $\begin{array}{c}\text { Median } \\
\text { Salary Index }\end{array}$ & $\begin{array}{c}\mathrm{BPS}^{+} \\
\text {Index }\end{array}$ & $\begin{array}{c}\text { Actual } \\
\text { CPI }\end{array}$ & $\begin{array}{l}\text { Adjusted } \\
\text { CPI }\end{array}$ \\
\hline 2008-2009 & 10 & 3,748 & $\$ 85,320$ & $\$ 48,108$ & 251.8 & 291.4 & 219.9 & 211.6 \\
\hline $2007-2008$ & 10 & 3,797 & 80,261 & 44,359 & 236.9 & 268.7 & 208.3 & 200.5 \\
\hline 2006-2007 & 10 & 3,832 & 80,124 & 42,765 & 236.5 & 259.1 & 203.5 & 195.9 \\
\hline 2005-2006 & 10 & 3,921 & 76,083 & 38,673 & 224.6 & 234.3 & 195.4 & 188.1 \\
\hline 2004-2005 & 10 & 3,946 & 74,022 & 34,764 & 218.5 & 210.6 & 189.4 & 182.3 \\
\hline 2003-2004 & 10 & 3,877 & 70,020 & 34,739 & 206.8 & 210.4 & 183.9 & 177.0 \\
\hline $2002-2003$ & 10 & 3,804 & 65,289 & 34,739 & 192.7 & 210.4 & 180.1 & 173.3 \\
\hline 2001-2002 & 10 & 3,717 & 65,025 & 34,389 & 191.9 & 208.3 & 177.5 & 170.8 \\
\hline $2000-2001$ & 10 & 3,731 & 62,521 & 31,774 & 184.5 & 192.5 & 172.8 & 166.3 \\
\hline 1999-2000 & 10 & 3,737 & 59,916 & 30,849 & 176.8 & 186.9 & 166.7 & 160.4 \\
\hline 1998-1999 & 11 & 3,819 & 56,000 & 29,877 & 165.3 & 181.0 & 163.2 & 157.1 \\
\hline 1997-1998 & 11 & 3,779 & 55,055 & 28,724 & 162.5 & 174.0 & 160.5 & 154.5 \\
\hline 1996-1997 & 11 & 3,799 & 51,150 & 28,380 & 151.0 & 172.0 & 157.0 & 151.1 \\
\hline 1995-1996 & 11 & 3,915 & 49,149 & 28,162 & 145.1 & 170.7 & 152.5 & 146.8 \\
\hline 1994-1995 & 11 & 3,837 & 47,997 & 27,813 & 141.7 & 168.6 & 148.4 & 142.8 \\
\hline 1993-1994 & 11 & 4,003 & 44,949 & 26,806 & 132.7 & 162.5 & 144.4 & 139.0 \\
\hline 1992-1993 & 11 & 4,172 & 43,876 & 23,500 & 129.6 & 142.4 & 140.2 & 134.9 \\
\hline 1991-1992 & 11 & 2,906 & 42,455 & 23,500 & 125.4 & 142.4 & 136.2 & 131.1 \\
\hline 1990-1991 & 12 & 1,363 & 36,013 & 20,800 & 106.3 & 126.1 & 130.7 & 125.8 \\
\hline 1989-1990 & 11 & 3,767 & 40,106 & 20,195 & 118.4 & 122.4 & 124.0 & 119.3 \\
\hline 1988-1989 & 11 & 3,781 & 37,544 & 19,100 & 110.9 & 115.8 & 118.3 & 113.9 \\
\hline 1987-1988 & 11 & 3,765 & 36,250 & 18,405 & 107.0 & 111.5 & 113.6 & 109.3 \\
\hline 1986-1987 & 10 & 2,790 & 33,020 & 17,912 & 97.5 & 108.6 & 109.6 & 105.5 \\
\hline 1985-1986 & 12 & 3,874 & 33,720 & 17,308 & 99.6 & 104.9 & 107.6 & 103.6 \\
\hline 1984-1985 & 11 & 3,840 & 33,863 & 16,500 & 100.0 & 100.0 & 103.9 & 100.0 \\
\hline
\end{tabular}




\title{
ARL UNIVERSITY LIBRARIES
}

\author{
TABLes 7-25
}




\section{Table 7: Filled Positions; Average, Median, Beginning Professional Salaries; and Average YeARS OF EXPERIENCE IN ARL University Libraries, FY 2008-2009}

\begin{tabular}{|c|c|c|c|c|c|c|c|c|}
\hline \multirow[b]{2}{*}{ Institution } & \multirow{2}{*}{$\begin{array}{c}\text { Filled } \\
\text { Positions } \\
\text { FY } 2009\end{array}$} & \multicolumn{2}{|c|}{$\begin{array}{l}\text { Average } \\
\text { Salaries }\end{array}$} & \multicolumn{2}{|c|}{$\begin{array}{l}\text { Median } \\
\text { Salaries }\end{array}$} & \multicolumn{2}{|c|}{$\begin{array}{c}\text { Beginning } \\
\text { Salaries }\end{array}$} & \multirow{2}{*}{$\begin{array}{l}\text { Average } \\
\text { Yrs. Exp } \\
\text { FY } 2009\end{array}$} \\
\hline & & FY 2008 & FY 2009 & FY 2008 & FY 2009 & FY 2008 & FY 2009 & \\
\hline Alabama $^{\ddagger}$ & 58 & $\$ 58,232$ & $\$ 58,655$ & $\$ 52,891$ & $\$ 52,205$ & $\$ 40,000$ & 42,000 & 14.7 \\
\hline Alberta $^{\dagger \neq}$ & 67 & 74,787 & 89,260 & 82,536 & 95,438 & 42,997 & 50,126 & 17.4 \\
\hline Arizona ${ }^{\ddagger}$ & 59 & 64,731 & 66,447 & 60,136 & 61,595 & 47,325 & 47,093 & 17.9 \\
\hline Arizona State ${ }^{\ddagger}$ & 70 & 64,325 & 63,110 & 62,333 & 61,000 & 43,000 & 43,000 & 16.5 \\
\hline Auburn ${ }^{\ddagger}$ & 46 & 61,104 & 60,231 & 58,540 & 55,290 & 44,720 & 44,720 & 15.6 \\
\hline Boston University & 58 & 54,472 & 59,236 & 51,450 & 55,200 & 40,800 & 42,300 & 16.5 \\
\hline Boston College $e^{\ddagger}$ & 63 & 67,847 & 69,672 & 65,293 & 67,184 & 41,050 & 42,300 & 18.4 \\
\hline Brigham Young ${ }^{\ddagger}$ & 114 & 61,929 & 63,925 & 60,200 & 62,400 & 46,000 & 48,000 & 17.7 \\
\hline British Columbia ${ }^{\ddagger \ddagger}$ & 89 & 69,414 & 79,910 & 68,737 & 80,769 & 45,784 & 53,021 & 14.6 \\
\hline Brown ${ }^{\ddagger}$ & 68 & 65,442 & 67,804 & 61,998 & 63,854 & 38,000 & 39,500 & 20.5 \\
\hline California, Berkeley ${ }^{\ddagger}$ & 103 & 80,749 & 82,952 & 79,108 & 80,374 & 40,008 & 46,164 & 18.3 \\
\hline California, Davis ${ }^{\ddagger}$ & 45 & 73,853 & 79,652 & 79,968 & 82,637 & 40,008 & 46,164 & 21 \\
\hline California, Irvine & 56 & 71,572 & 76,045 & 73,356 & 75,708 & 40,008 & 46,164 & 15.3 \\
\hline California, Los Angeles ${ }^{\ddagger}$ & 154 & 76,029 & 75,812 & 73,382 & 73,799 & 40,008 & 46,164 & 15.1 \\
\hline California, Riverside ${ }^{\ddagger}$ & 53 & 69,120 & 74,166 & 68,000 & 74,400 & 42,204 & 47,087 & 20.1 \\
\hline California, San Diego ${ }^{\ddagger}$ & 87 & 74,176 & 76,016 & 73,356 & 75,708 & 40,008 & 46,164 & 16.4 \\
\hline California, Santa Barbara ${ }^{*}$ & 60 & 70,227 & 71,466 & 66,756 & 68,892 & 42,008 & 46,164 & 16.8 \\
\hline Case Western Reserve ${ }^{\ddagger}$ & 39 & 57,089 & 57,972 & 54,335 & 54,389 & 35,000 & 35,000 & 15 \\
\hline Chicago $^{\ddagger}$ & 71 & 72,179 & 74,480 & 68,418 & 70,290 & 46,800 & 48,204 & 18.4 \\
\hline Cincinnati $^{\ddagger}$ & 50 & 65,283 & 66,847 & 61,552 & 61,332 & 38,000 & 39,000 & 22.1 \\
\hline Colorado $^{\ddagger}$ & 42 & 65,510 & 66,826 & 61,431 & 64,388 & 41,000 & 44,000 & 16.7 \\
\hline Colorado State ${ }^{\ddagger}$ & 52 & 62,333 & 68,355 & 60,650 & 65,300 & 43,500 & 45,400 & 16.1 \\
\hline Columbia & 189 & 70,838 & 72,352 & 61,585 & 64,265 & 51,500 & 51,500 & 15.3 \\
\hline Connecticut ${ }^{\ddagger}$ & 61 & 76,946 & 79,855 & 74,661 & 78,680 & 48,000 & 48,000 & 18 \\
\hline Cornell ${ }^{\ddagger}$ & 112 & 68,228 & 69,564 & 62,490 & 63,600 & 45,000 & 47,000 & 16 \\
\hline Dartmouth ${ }^{\ddagger}$ & 51 & 71,642 & 72,539 & 71,597 & 69,536 & 40,000 & 42,500 & 17.3 \\
\hline Delaware & 58 & 69,033 & 72,792 & 64,235 & 71,037 & 43,600 & 43,600 & 17.2 \\
\hline Duke ${ }^{\ddagger}$ & 143 & 60,715 & 63,058 & 55,650 & 58,583 & 43,000 & 44,000 & 15.2 \\
\hline Emory ${ }^{\ddagger}$ & 72 & 64,551 & 69,025 & 61,000 & 63,653 & 38,000 & 41,500 & 15.3 \\
\hline Florida ${ }^{\ddagger}$ & 68 & 56,403 & 58,974 & 52,327 & 55,045 & 42,000 & 42,000 & 14.4 \\
\hline Florida State ${ }^{\ddagger}$ & 54 & 48,656 & 49,803 & 45,752 & 47,000 & 42,000 & 42,000 & 13.6 \\
\hline George Washington & 43 & 64,950 & 70,759 & 59,401 & 65,328 & 42,000 & 47,000 & 15 \\
\hline Georgetown ${ }^{\ddagger}$ & 53 & 66,689 & 69,739 & 61,508 & 63,538 & 45,000 & 45,000 & 19.9 \\
\hline Georgia ${ }^{\ddagger}$ & 74 & 57,082 & 57,628 & 49,634 & 51,005 & 35,000 & 38,000 & 16.3 \\
\hline Georgia Tech ₹ & 42 & 58,753 & 60,944 & 54,631 & 56,270 & 42,000 & 42,000 & 16.2 \\
\hline Guelph $^{\dagger \neq}$ & 47 & 62,069 & 77,319 & 58,271 & 71,928 & 38,590 & 57,242 & 17.8 \\
\hline Harvard ${ }^{\ddagger}$ & 469 & 72,861 & 78,019 & 67,486 & 70,900 & 47,100 & 48,800 & 15.8 \\
\hline Hawaii ${ }^{\ddagger}$ & 79 & 61,330 & 67,491 & 60,354 & 67,391 & 42,026 & 42,649 & 15.8 \\
\hline Houston ${ }^{\ddagger}$ & 35 & 56,608 & 63,512 & 49,037 & 62,480 & 40,000 & 42,000 & 15.1 \\
\hline Howard ${ }^{\ddagger}$ & 31 & 53,211 & 53,741 & 53,359 & 53,440 & 35,049 & 36,000 & 25.9 \\
\hline Illinois, Chicago ${ }^{\ddagger}$ & 50 & 58,925 & 61,206 & 54,885 & 56,457 & 47,000 & 47,000 & 17.5 \\
\hline Illinois, Urbana ${ }^{\ddagger}$ & 145 & 64,009 & 67,213 & 58,247 & 62,036 & 44,500 & 46,000 & 13.7 \\
\hline Indiana ${ }^{\ddagger}$ & 81 & 64,257 & 63,786 & 59,705 & 60,011 & 40,000 & 40,400 & 18.5 \\
\hline Iowa ${ }^{\ddagger}$ & 70 & 63,234 & 63,196 & 55,700 & 56,796 & 40,000 & 41,000 & 17 \\
\hline Iowa State ${ }^{\ddagger}$ & 55 & 59,891 & 61,863 & 57,452 & 59,887 & 42,500 & 43,000 & 18.5 \\
\hline Johns Hopkins ${ }^{\ddagger}$ & 79 & 65,765 & 67,265 & 60,060 & 63,702 & 48,335 & 50,026 & 16.2 \\
\hline Kansas & 83 & 59,070 & 61,056 & 55,120 & 56,200 & 40,000 & 49,000 & 15.2 \\
\hline Kent State $^{\ddagger}$ & 63 & 56,086 & 57,317 & 53,777 & 53,777 & 43,389 & 43,389 & 16 \\
\hline Kentucky ${ }^{\ddagger}$ & 63 & 59,613 & 60,909 & 57,817 & 59,697 & 38,500 & 41,000 & 21.3 \\
\hline Laval ${ }^{\dagger \ddagger}$ & 64 & 59,630 & 68,511 & 62,715 & 69,901 & 40,025 & 47,613 & 15.3 \\
\hline Louisiana State ${ }^{\ddagger}$ & 49 & 48,711 & 51,839 & 44,879 & 47,715 & 38,000 & 38,000 & 14.9 \\
\hline Louisville ${ }^{\ddagger}$ & 34 & 65,230 & 61,851 & 61,986 & 56,845 & 37,000 & 37,000 & 19.4 \\
\hline $\mathrm{McGill}^{+}$ & 64 & 66,010 & 73,148 & 64,728 & 67,233 & 40,625 & 49,500 & 16.9 \\
\hline McMaster $^{+}$ & 32 & 67,549 & 78,776 & 69,173 & 78,858 & 38,598 & 44,771 & 17.7 \\
\hline Manitoba $^{+\neq}$ & 42 & 77,261 & 89,270 & 79,159 & 93,351 & 41,331 & 46,970 & 21.9 \\
\hline Maryland & 82 & 64,926 & 67,924 & 61,930 & 66,419 & 42,000 & 42,000 & 19.5 \\
\hline Massachusetts ${ }^{\ddagger}$ & 59 & 69,319 & 70,508 & 69,746 & 72,263 & 40,730 & 42,155 & 16.2 \\
\hline MIT & 95 & 69,719 & 75,733 & 65,650 & 70,900 & 48,000 & 51,000 & 15 \\
\hline Miami & 46 & 65,171 & 69,165 & 60,000 & 64,935 & 45,000 & 45,000 & 17.2 \\
\hline
\end{tabular}




\section{Table 7: Filled Positions; Average, Median, Beginning Professional Salaries; and Average YeARs of EXPerience in ARL University Libraries, FY 2008-2009}

\begin{tabular}{|c|c|c|c|c|c|c|c|c|}
\hline \multirow[b]{2}{*}{ Institution } & \multirow{2}{*}{$\begin{array}{c}\text { Filled } \\
\text { Positions } \\
\text { FY } 2009\end{array}$} & \multicolumn{2}{|c|}{$\begin{array}{l}\text { Average } \\
\text { Salaries }\end{array}$} & \multicolumn{2}{|c|}{$\begin{array}{l}\text { Median } \\
\text { Salaries }\end{array}$} & \multicolumn{2}{|c|}{$\begin{array}{c}\text { Beginning } \\
\text { Salaries }\end{array}$} & \multirow{2}{*}{$\begin{array}{l}\text { Average } \\
\text { Yrs. Exp } \\
\text { FY } 2009\end{array}$} \\
\hline & & FY 2008 & FY 2009 & FY 2008 & FY 2009 & FY 2008 & FY 2009 & \\
\hline Michigan $^{\ddagger}$ & 116 & 66,608 & 70,031 & 63,413 & 65,564 & 41,000 & 42,000 & 18.8 \\
\hline Michigan State ${ }^{\ddagger}$ & 72 & 61,743 & 63,867 & 57,000 & 60,000 & 47,000 & 47,000 & 15.8 \\
\hline Minnesota & 110 & 66,067 & 67,975 & 63,532 & 65,446 & 40,000 & 40,000 & 18.2 \\
\hline Missouri $^{\ddagger}$ & 37 & 57,607 & 55,264 & 50,442 & 48,854 & 38,500 & 40,000 & 20 \\
\hline Montreal $^{+\ddagger}$ & 90 & 60,262 & 69,342 & 58,216 & 66,631 & 36,144 & 41,738 & 15.4 \\
\hline Nebraska $^{\ddagger}$ & 46 & 61,376 & 65,751 & 54,624 & 57,671 & 45,000 & 50,000 & 20.1 \\
\hline New Mexico ${ }^{\ddagger}$ & 34 & 76,612 & 78,591 & 74,768 & 76,307 & 40,000 & 40,000 & 20.9 \\
\hline New York University ${ }^{\ddagger}$ & 55 & 77,595 & 78,920 & 68,882 & 70,769 & 51,000 & 52,000 & 18.4 \\
\hline North Carolina ${ }^{\ddagger}$ & 104 & 67,938 & 67,177 & 62,825 & 63,750 & 42,000 & 44,000 & 17.6 \\
\hline North Carolina State ${ }^{\ddagger}$ & 84 & 68,461 & 70,667 & 61,717 & 64,895 & 47,000 & 49,000 & 10.4 \\
\hline Northwestern ${ }^{\ddagger}$ & 76 & 65,234 & 66,328 & 61,203 & 62,847 & 39,000 & 42,000 & 17.3 \\
\hline Notre Dame ${ }^{\ddagger}$ & 59 & 66,827 & 68,224 & 65,769 & 65,738 & 40,000 & 40,000 & 17.4 \\
\hline Ohio University ${ }^{\ddagger}$ & 45 & 48,853 & 54,210 & 45,010 & 48,170 & 36,000 & 41,500 & 12.6 \\
\hline Ohio State ${ }^{\ddagger}$ & 134 & 56,745 & 57,439 & 52,941 & 52,432 & 41,500 & 43,000 & 14.2 \\
\hline Oklahoma ${ }^{\ddagger}$ & 45 & 53,241 & 54,228 & 50,823 & 52,228 & 40,000 & 42,000 & 14.2 \\
\hline Oklahoma State ${ }^{\ddagger}$ & 62 & 54,593 & 56,703 & 49,824 & 53,640 & 36,000 & 36,000 & 17.4 \\
\hline Oregon ${ }^{\ddagger}$ & 56 & 54,999 & 56,965 & 51,176 & 53,792 & 35,000 & 40,000 & 15.3 \\
\hline Pennsylvania ${ }^{\ddagger}$ & 97 & 63,029 & 65,914 & 58,526 & 61,122 & 42,500 & 42,500 & 9 \\
\hline Pennsylvania State ${ }^{\ddagger}$ & 134 & 66,725 & 69,421 & 62,730 & 65,556 & 41,200 & 42,436 & 19.4 \\
\hline Pittsburgh ${ }^{\ddagger}$ & 63 & 64,047 & 66,544 & 58,451 & 59,984 & 34,000 & 34,000 & 17.9 \\
\hline Princeton ${ }^{\ddagger}$ & 115 & 74,179 & 77,939 & 70,000 & 72,150 & 45,000 & 50,000 & 21.2 \\
\hline Purdue ${ }^{\ddagger}$ & 68 & 65,312 & 66,957 & 57,815 & 61,000 & 43,500 & 45,000 & 17.5 \\
\hline Queen`s ${ }^{\dagger \neq}$ & 34 & 72,268 & 88,107 & 74,078 & 89,828 & 40,695 & 49,500 & 19.6 \\
\hline Rice & 59 & 61,662 & 61,433 & 54,900 & 55,450 & 39,060 & 38,300 & 15.2 \\
\hline Rochester ${ }^{\ddagger}$ & 76 & 55,411 & 55,965 & 51,282 & 52,540 & 36,700 & 38,168 & 14.7 \\
\hline Rutgers ${ }^{\ddagger}$ & 82 & 82,023 & 88,899 & 84,829 & 92,106 & 46,781 & 47,390 & 21.2 \\
\hline Saskatchewan ${ }^{\dagger \neq}$ & 39 & 74,526 & 85,401 & 74,456 & 85,977 & 41,598 & 49,600 & 18 \\
\hline South Carolina ${ }^{\ddagger}$ & 53 & 51,457 & 53,670 & 47,983 & 49,838 & 34,000 & 34,000 & 17.1 \\
\hline Southern California ${ }^{*}$ & 106 & 70,463 & 73,788 & 64,335 & 66,944 & 45,000 & 48,500 & 17.9 \\
\hline Southern Illinois & 40 & 54,562 & 57,003 & 49,375 & 49,117 & 41,000 & 43,000 & 14.5 \\
\hline SUNY Albany ${ }^{\ddagger}$ & 60 & 60,304 & 67,251 & 56,625 & 59,704 & 38,000 & 38,500 & 16.6 \\
\hline SUNY Buffalo ${ }^{\ddagger}$ & 79 & 64,414 & 69,982 & 60,852 & 66,684 & 45,000 & 45,000 & 19.7 \\
\hline SUNY Stony Brook ${ }^{\ddagger}$ & 30 & 70,027 & 75,948 & 67,828 & 73,259 & 43,000 & 44,000 & 21.9 \\
\hline Syracuse ${ }^{\ddagger}$ & 49 & 65,131 & 65,441 & 58,263 & 61,493 & & $\mathrm{~N} / \mathrm{A}$ & 18.7 \\
\hline Temple ${ }^{\ddagger}$ & 39 & 65,552 & 66,114 & 56,572 & 56,020 & 39,000 & 42,744 & 18.7 \\
\hline Tennessee ${ }^{\ddagger}$ & 40 & 68,333 & 70,344 & 65,394 & 67,823 & 42,000 & 44,000 & 18.5 \\
\hline Texas ${ }^{\ddagger}$ & 123 & 63,467 & 66,554 & 57,809 & 58,958 & 45,000 & 45,000 & 16.4 \\
\hline Texas A\&M ${ }^{\ddagger}$ & 138 & 60,024 & 59,250 & 54,857 & 54,156 & 44,000 & 47,000 & 14.9 \\
\hline Texas Tech ${ }^{\ddagger}$ & 51 & 59,987 & 60,494 & 53,079 & 55,036 & 45,000 & 45,000 & 12.3 \\
\hline Toronto ${ }^{+\ddagger}$ & 144 & 76,710 & 90,829 & 78,443 & 91,773 & 43,010 & 51,381 & 17 \\
\hline Tulane & 32 & 59,023 & 62,896 & 55,664 & 60,000 & 37,000 & 40,000 & 18.3 \\
\hline Utah $^{\ddagger}$ & 57 & 59,993 & 61,119 & 54,614 & 57,250 & 42,000 & 44,000 & 18.7 \\
\hline Vanderbilt ${ }^{*}$ & 57 & 57,775 & 59,810 & 52,479 & 54,666 & 39,000 & 40,000 & 17.2 \\
\hline Virginia $^{\ddagger}$ & 67 & 67,602 & 69,246 & 60,000 & 60,450 & 43,000 & 44,000 & 16.7 \\
\hline Virginia Tech & 35 & 62,691 & 65,516 & 59,672 & 62,556 & 38,500 & 41,000 & 18.6 \\
\hline Washington $^{\ddagger}$ & 117 & 63,655 & 66,517 & 58,020 & 60,312 & 41,000 & 42,600 & 19.2 \\
\hline Washington State ${ }^{\ddagger}$ & 48 & 58,249 & 59,820 & 54,475 & 56,482 & 38,000 & 38,500 & 17.3 \\
\hline Washington U.-St. Louis ${ }^{\ddagger}$ & 61 & 58,142 & 59,497 & 51,727 & 54,468 & 37,000 & 40,000 & 15.8 \\
\hline Waterloo $^{+\neq}$ & 34 & 68,405 & 77,432 & 70,072 & 78,311 & 41,683 & 49,090 & 18.4 \\
\hline Wayne State ${ }^{\ddagger}$ & 98 & 53,075 & 54,962 & 47,315 & 50,031 & 40,500 & 40,500 & 17 \\
\hline Western Ontario $^{+\neq}$ & 63 & 58,941 & 68,168 & 54,246 & 63,872 & 46,000 & 46,906 & 13.4 \\
\hline Wisconsin ${ }^{\ddagger}$ & 153 & 59,685 & 60,304 & 57,766 & 57,808 & 39,338 & 40,526 & 16.6 \\
\hline Yale $^{+\ddagger}$ & 202 & 77,131 & 82,622 & 72,263 & 76,974 & 49,500 & 50,500 & 18.9 \\
\hline York ${ }^{+}$ & 55 & 81,659 & 95,755 & 76,495 & 88,458 & 43,275 & 48,510 & 16.7 \\
\hline
\end{tabular}

Directors are included in figures for average years of experience and filled positions, but not in either the average or median salary statistics. Excludes medical and law libraries. See Tables 35 and 42 for statistics related to medical and law library salaries.

+ Canadian salaries are expressed in US dollars.

‡ See Footnotes. 


\section{Table 8: Beginning Professional Salaries in ARL University Libraries RANK ORder TABLe, FY 2007-2008}

\begin{tabular}{|c|c|c|c|c|c|}
\hline Rank & Institution & Salary & Rank & Institution & Salary \\
\hline 1 & Columbia & 51,500 & 58 & Colorado & 41,000 \\
\hline 2 & New York & 51,000 & 58 & Michigan & 41,000 \\
\hline 3 & Yale & 49,500 & 58 & Southern Illinois & 41,000 \\
\hline 4 & Johns Hopkins & 48,335 & 58 & Washington & 41,000 \\
\hline 5 & Connecticut & 48,000 & 62 & Boston University & 40,800 \\
\hline 5 & MIT & 48,000 & 63 & Massachusetts & 40,730 \\
\hline 7 & Arizona & 47,325 & 64 & Queen's & 40695 \\
\hline 8 & Harvard & 47,100 & 65 & McGill & 40,625 \\
\hline 9 & Illinois, Chicago & 47,000 & 65 & Western Ontario & 40,625 \\
\hline 9 & Michigan State & 47,000 & 67 & Wayne State & 40,500 \\
\hline 9 & North Carolina State & 47,000 & 68 & Laval & 40,025 \\
\hline 12 & Chicago & 46,800 & 69 & Calif. Berkeley & 40,008 \\
\hline 13 & Rutgers & 46,781 & 69 & Calif. Davis & 40,008 \\
\hline 14 & Brigham Young & 46,000 & 69 & Calif. Irvine & 40,008 \\
\hline 15 & British Columbia & 45,784 & 69 & Calif. Los Angeles & 40,008 \\
\hline 16 & Cornell & 45,000 & 69 & Calif. San Diego & 40,008 \\
\hline 16 & Georgetown & 45,000 & 74 & Alabama & 40,000 \\
\hline 16 & Miami & 45,000 & 74 & Dartmouth & 40,000 \\
\hline 16 & Nebraska & 45,000 & 74 & Houston & 40,000 \\
\hline 16 & Princeton & 45,000 & 74 & Indiana & 40,000 \\
\hline 16 & Southern California & 45,000 & 74 & Iowa & 40,000 \\
\hline 16 & SUNY Buffalo & 45,000 & 74 & Kansas & 40,000 \\
\hline 16 & Texas & 45,000 & 74 & Minnesota & 40,000 \\
\hline 16 & Texas Tech & 45,000 & 74 & New Mexico & 40,000 \\
\hline 25 & Auburn & 44,720 & 74 & Notre Dame & 40,000 \\
\hline 26 & Illinois, Urbana & 44,500 & 74 & Oklahoma & 40,000 \\
\hline 27 & Texas A\&M & 44,000 & 84 & Wisconsin & 39,338 \\
\hline 28 & Delaware & 43,600 & 85 & Rice & 39,060 \\
\hline 29 & Colorado State & 43,500 & 86 & Northwestern & 39,000 \\
\hline 29 & Purdue & 43,500 & 86 & Temple & 39,000 \\
\hline 31 & Kent State & 43,389 & 86 & Vanderbilt & 39,000 \\
\hline 32 & York & 43,275 & 89 & McMaster & 38598 \\
\hline 33 & Toronto & 43010 & 90 & Guelph & 38,590 \\
\hline 34 & Arizona State & 43,000 & 91 & Kentucky & 38,500 \\
\hline 34 & Duke & 43,000 & 91 & Missouri & 38,500 \\
\hline 34 & SUNY Stony Brook & 43,000 & 91 & Virginia Tech & 38,500 \\
\hline 34 & Virginia & 43,000 & 94 & Brown & 38,000 \\
\hline 38 & Alberta & 42,997 & 94 & Cincinnati & 38,000 \\
\hline 39 & Iowa State & 42,500 & 94 & Emory & 38,000 \\
\hline 39 & Pennsylvania & 42,500 & 94 & Louisiana State & 38,000 \\
\hline 41 & Calif. Riverside & 42,204 & 94 & SUNY Albany & 38,000 \\
\hline 42 & Hawaii & 42,026 & 94 & Washington State & 38,000 \\
\hline 43 & Calif. Santa Barbara & 42,008 & 100 & Louisville & 37,000 \\
\hline 44 & Florida & 42,000 & 100 & Tulane & 37,000 \\
\hline 44 & Florida State & 42,000 & 100 & Washington-St. Louis & 37,000 \\
\hline 44 & George Washington & 42,000 & 103 & Rochester & 36,700 \\
\hline 44 & Georgia Tech & 42,000 & 104 & Montreal & 36144 \\
\hline 44 & Maryland & 42,000 & 105 & Ohio & 36,000 \\
\hline 44 & North Carolina & 42,000 & 105 & Oklahoma State & 36,000 \\
\hline 44 & Tennessee & 42,000 & 107 & Howard & 35,049 \\
\hline 44 & Utah & 42,000 & 108 & Case Western Reserve & 35,000 \\
\hline 52 & Waterloo & 41,683 & 108 & Georgia & 35,000 \\
\hline 53 & Saskatchewan & 41,598 & 108 & Oregon & 35,000 \\
\hline 54 & Ohio State & 41,500 & 111 & Pittsburgh & 34,000 \\
\hline 55 & Manitoba & 41,331 & 111 & South Carolina & 34,000 \\
\hline 56 & Pennsylvania State & 41,200 & 113 & Syracuse & $\mathrm{N} / \mathrm{A}$ \\
\hline 57 & Boston College & 41,050 & & & \\
\hline
\end{tabular}

Reprinted from ARL Annual Salary Survey 2007-2008. Beginning salary figures represent officially designated base, not necessarily salaries of actual incumbents. Excludes medical and law libraries. See Tables 36 and 43 for statistics related to medical and law library salaries. Canadian salaries are expressed in US dollars. 


\section{Table 9: Beginning Professional Salaries in ARL University Libraries RANK ORDER TABLE, FY 2008-2009}

\begin{tabular}{|c|c|c|c|c|c|}
\hline Rank & Institution & Salary & Rank & Institution & Salary \\
\hline 1 & Guelph & 57,242 & 58 & Delaware & 43,600 \\
\hline 2 & British Columbia & 53,021 & 59 & Kent State & 43,389 \\
\hline 3 & New York & 52,000 & 60 & Arizona State & 43,000 \\
\hline 4 & Columbia & 51,500 & 60 & Iowa State & 43,000 \\
\hline 5 & Toronto & 51,381 & 60 & Ohio State & 43,000 \\
\hline 6 & MIT & 51,000 & 60 & Southern Illinois & 43,000 \\
\hline 7 & Yale & 50,500 & 64 & Temple & 42,744 \\
\hline 8 & Alberta & 50,126 & 65 & Hawaii & 42,649 \\
\hline 9 & Johns Hopkins & 50,026 & 66 & Washington & 42,600 \\
\hline 10 & Nebraska & 50,000 & 67 & Dartmouth & 42,500 \\
\hline 10 & Princeton & 50,000 & 67 & Pennsylvania & 42,500 \\
\hline 12 & Saskatchewan & 49,600 & 69 & Pennsylvania State & 42,436 \\
\hline 13 & McGill & 49,500 & 70 & Boston University & 42,300 \\
\hline 13 & Queen's & 49,500 & 70 & Boston College & 42,300 \\
\hline 15 & Waterloo & 49,090 & 72 & Massachusetts & 42,155 \\
\hline 16 & Kansas & 49,000 & 73 & Alabama & 42,000 \\
\hline 16 & North Carolina State & 49,000 & 73 & Florida & 42,000 \\
\hline 18 & Harvard & 48,800 & 73 & Florida State & 42,000 \\
\hline 19 & York & 48,510 & 73 & Georgia Tech & 42,000 \\
\hline 20 & Southern California & 48,500 & 73 & Houston & 42,000 \\
\hline 21 & Chicago & 48,204 & 73 & Maryland & 42,000 \\
\hline 22 & Brigham Young & 48,000 & 73 & Michigan & 42,000 \\
\hline 22 & Connecticut & 48,000 & 73 & Northwestern & 42,000 \\
\hline 24 & Laval & 47,613 & 73 & Oklahoma & 42,000 \\
\hline 25 & Rutgers & 47,390 & 82 & Montreal & 41,738 \\
\hline 26 & Arizona & 47,093 & 83 & Emory & 41,500 \\
\hline 27 & Calif. Riverside & 47,087 & 83 & Ohio & 41,500 \\
\hline 28 & Cornell & 47,000 & 85 & Iowa & 41,000 \\
\hline 28 & George Washington & 47,000 & 85 & Kentucky & 41,000 \\
\hline 28 & Illinois, Chicago & 47,000 & 85 & Virginia Tech & 41,000 \\
\hline 28 & Michigan State & 47,000 & 88 & Wisconsin & 40,526 \\
\hline 28 & Texas A\&M & 47,000 & 89 & Wayne State & 40,500 \\
\hline 33 & Manitoba & 46,970 & 90 & Indiana & 40,400 \\
\hline 34 & Western Ontario & 46,906 & 91 & Minnesota & 40,000 \\
\hline 35 & Calif. Berkeley & 46,164 & 91 & Missouri & 40,000 \\
\hline 35 & Calif. Davis & 46,164 & 91 & New Mexico & 40,000 \\
\hline 35 & Calif. Irvine & 46,164 & 91 & Notre Dame & 40,000 \\
\hline 35 & Calif. Los Angeles & 46,164 & 91 & Oregon & 40,000 \\
\hline 35 & Calif. San Diego & 46,164 & 91 & Tulane & 40,000 \\
\hline 35 & Calif. Santa Barbara & 46,164 & 91 & Vanderbilt & 40,000 \\
\hline 41 & Illinois, Urbana & 46,000 & 91 & Washington-St. Louis & 40,000 \\
\hline 42 & Colorado State & 45,400 & 99 & Brown & 39,500 \\
\hline 43 & Georgetown & 45,000 & 100 & Cincinnati & 39,000 \\
\hline 43 & Miami & 45,000 & 101 & SUNY Albany & 38,500 \\
\hline 43 & Purdue & 45,000 & 101 & Washington State & 38,500 \\
\hline 43 & SUNY Buffalo & 45,000 & 103 & Rice & 38,300 \\
\hline 43 & Texas & 45,000 & 104 & Rochester & 38,168 \\
\hline 43 & Texas Tech & 45,000 & 105 & Georgia & 38,000 \\
\hline 49 & McMaster & 44,771 & 105 & Louisiana State & 38,000 \\
\hline 50 & Auburn & 44,720 & 107 & Louisville & 37,000 \\
\hline 51 & Colorado & 44,000 & 108 & Howard & 36,000 \\
\hline 51 & Duke & 44,000 & 108 & Oklahoma State & 36,000 \\
\hline 51 & North Carolina & 44,000 & 110 & Case Western Reserve & 35,000 \\
\hline 51 & SUNY Stony Brook & 44,000 & 111 & Pittsburgh & 34,000 \\
\hline 51 & Tennessee & 44,000 & 111 & South Carolina & 34,000 \\
\hline 51 & Utah & 44,000 & 113 & Syracuse & N/A \\
\hline 51 & Virginia & 44,000 & & & \\
\hline
\end{tabular}

Beginning salary figures represent officially designated base, not necessarily salaries of actual incumbents. Excludes medical and law libraries. See Tables 36 and 43 for statistics related to medical and law library salaries.

Canadian salaries are expressed in US dollars. 


\section{Table 10: Median Professional Salaries in ARL University Libraries RANK ORder TABLE, FY 2007-2008}

\begin{tabular}{|c|c|c|c|c|c|}
\hline Rank & Institution & Salary & Rank & Institution & Salary \\
\hline 1 & Rutgers & 84,829 & 58 & Miami & 60,000 \\
\hline 2 & Alberta & 82,536 & 58 & Virginia & 60,000 \\
\hline 3 & California, Davis & 79,968 & 60 & Indiana & 59,705 \\
\hline 4 & Manitoba & 79,159 & 61 & Virginia Tech & 59,672 \\
\hline 5 & California, Berkeley & 79,108 & 62 & George Washington & 59,401 \\
\hline 6 & Toronto & 78,443 & 63 & Auburn & 58,540 \\
\hline 7 & York & 76,495 & 64 & Pennsylvania & 58,526 \\
\hline 8 & New Mexico & 74,768 & 65 & Pittsburgh & 58,451 \\
\hline 9 & Connecticut & 74,661 & 66 & Guelph & 58,271 \\
\hline 10 & Saskatchewan & 74,456 & 67 & Syracuse & 58,263 \\
\hline 11 & Queen`s & 74,078 & 68 & Illinois, Urbana & 58,247 \\
\hline 12 & California, Los Angeles & 73,382 & 69 & Montreal & 58,216 \\
\hline 13 & California, Irvine & 73,356 & 70 & Washington & 58,020 \\
\hline 13 & California, San Diego & 73,356 & 71 & Kentucky & 57,817 \\
\hline 15 & Yale & 72,263 & 72 & Purdue & 57,815 \\
\hline 16 & Dartmouth & 71,597 & 73 & Texas & 57,809 \\
\hline 17 & Waterloo & 70,072 & 74 & Wisconsin & 57,766 \\
\hline 18 & Princeton & 70,000 & 75 & Iowa State & 57,452 \\
\hline 19 & Massachusetts & 69,746 & 76 & Michigan State & 57,000 \\
\hline 20 & McMaster & 69,173 & 77 & SUNY Albany & 56,625 \\
\hline 21 & New York University & 68,882 & 78 & Temple & 56,572 \\
\hline 22 & British Columbia & 68,737 & 79 & Iowa & 55,700 \\
\hline 23 & Chicago & 68,418 & 80 & Tulane & 55,664 \\
\hline 24 & California, Riverside & 68,000 & 81 & Duke & 55,650 \\
\hline 25 & SUNY Stony Brook & 67,828 & 82 & Kansas & 55,120 \\
\hline 26 & Harvard & 67,486 & 83 & Rice & 54,900 \\
\hline 27 & California, Santa Barbara & 66,756 & 84 & Illinois, Chicago & 54,885 \\
\hline 28 & Notre Dame & 65,769 & 85 & Texas A\&M & 54,857 \\
\hline 29 & MIT & 65,650 & 86 & Georgia Tech & 54,631 \\
\hline 30 & Tennessee & 65,394 & 87 & Nebraska & 54,624 \\
\hline 31 & Boston College & 65,293 & 88 & Utah & 54,614 \\
\hline 32 & McGill & 64,728 & 89 & Washington State & 54,475 \\
\hline 33 & Southern California & 64,335 & 90 & Case Western Reserve & 54,335 \\
\hline 34 & Delaware & 64,235 & 91 & Western Ontario & 54,246 \\
\hline 35 & Minnesota & 63,532 & 92 & Kent State & 53,777 \\
\hline 36 & Michigan & 63,413 & 93 & Howard & 53,359 \\
\hline 37 & North Carolina & 62,825 & 94 & Texas Tech & 53,079 \\
\hline 38 & Pennsylvania State & 62,730 & 95 & Ohio State & 52,941 \\
\hline 39 & Laval & 62,715 & 96 & Alabama & 52,891 \\
\hline 40 & Cornell & 62,490 & 97 & Vanderbilt & 52,479 \\
\hline 41 & Arizona State & 62,333 & 98 & Florida & 52,327 \\
\hline 42 & Brown & 61,998 & 99 & Washington U.-St .Louis & 51,727 \\
\hline 43 & Louisville & 61,986 & 100 & Boston University & 51,450 \\
\hline 44 & Maryland & 61,930 & 101 & Rochester & 51,282 \\
\hline 45 & North Carolina State & 61,717 & 102 & Oregon & 51,176 \\
\hline 46 & Columbia & 61,585 & 103 & Oklahoma & 50,823 \\
\hline 47 & Cincinnati & 61,552 & 104 & Missouri & 50,442 \\
\hline 48 & Georgetown & 61,508 & 105 & Oklahoma State & 49,824 \\
\hline 49 & Colorado & 61,431 & 106 & Georgia & 49,634 \\
\hline 50 & Northwestern & 61,203 & 107 & Southern Illinois & 49,375 \\
\hline 51 & Emory & 61,000 & 108 & Houston & 49,037 \\
\hline 52 & SUNY Buffalo & 60,852 & 109 & South Carolina & 47,983 \\
\hline 53 & Colorado State & 60,650 & 110 & Wayne State & 47,315 \\
\hline 54 & Hawaii & 60,354 & 111 & Florida State & 45,752 \\
\hline 55 & Brigham Young & 60,200 & 112 & Ohio University & 45,010 \\
\hline 56 & Arizona & 60,136 & 113 & Louisiana State & 44,879 \\
\hline 57 & Johns Hopkins & 60,060 & & & \\
\hline
\end{tabular}

Reprinted from ARL Annual Salary Survey 2007-2008. Salaries of directors are not included in the calculation of medians. Excludes medical and law libraries. See Tables 37 and 44 for statistics related to medical and law library salaries.

Canadian salaries are expressed in US dollars. 


\section{Table 11: Median Professional Salaries in ARL University Libraries RANK ORDER TABLE, FY 2008-2009}

\begin{tabular}{|c|c|c|c|c|c|}
\hline Rank & Institution & Salary & Rank & Institution & Salary \\
\hline 1 & Alberta & 95,438 & 58 & Virginia Tech & 62,556 \\
\hline 2 & Manitoba & 93,351 & 59 & Houston & 62,480 \\
\hline 3 & Rutgers & 92,106 & 60 & Brigham Young & 62,400 \\
\hline 4 & Toronto & 91,773 & 61 & Illinois, Urbana & 62,036 \\
\hline 5 & Queen`s & 89,828 & 62 & Arizona & 61,595 \\
\hline 6 & York & 88,458 & 63 & Syracuse & 61,493 \\
\hline 7 & Saskatchewan & 85,977 & 64 & Cincinnati & 61,332 \\
\hline 8 & California, Davis & 82,637 & 65 & Pennsylvania & 61,122 \\
\hline 9 & British Columbia & 80,769 & 66 & Arizona State & 61,000 \\
\hline 10 & California, Berkeley & 80,374 & 66 & Purdue & 61,000 \\
\hline 11 & McMaster & 78,858 & 68 & Virginia & 60,450 \\
\hline 12 & Connecticut & 78,680 & 69 & Washington & 60,312 \\
\hline 13 & Waterloo & 78,311 & 70 & Indiana & 60,011 \\
\hline 14 & Yale & 76,974 & 71 & Michigan State & 60,000 \\
\hline 15 & New Mexico & 76,307 & 71 & Tulane & 60,000 \\
\hline 16 & California, Irvine & 75,708 & 73 & Pittsburgh & 59,984 \\
\hline 16 & California, San Diego & 75,708 & 74 & Iowa State & 59,887 \\
\hline 18 & California, Riverside & 74,400 & 75 & SUNY Albany & 59,704 \\
\hline 19 & California, Los Angeles & 73,799 & 76 & Kentucky & 59,697 \\
\hline 20 & SUNY Stony Brook & 73,259 & 77 & Texas & 58,958 \\
\hline 21 & Massachusetts & 72,263 & 78 & Duke & 58,583 \\
\hline 22 & Princeton & 72,150 & 79 & Wisconsin & 57,808 \\
\hline 23 & Guelph & 71,928 & 80 & Nebraska & 57,671 \\
\hline 24 & Delaware & 71,037 & 81 & Utah & 57,250 \\
\hline 25 & Harvard & 70,900 & 82 & Louisville & 56,845 \\
\hline 25 & MIT & 70,900 & 83 & Iowa & 56,796 \\
\hline 27 & New York University & 70,769 & 84 & Washington State & 56,482 \\
\hline 28 & Chicago & 70,290 & 85 & Illinois, Chicago & 56,457 \\
\hline 29 & Laval & 69,901 & 86 & Georgia Tech & 56,270 \\
\hline 30 & Dartmouth & 69,536 & 87 & Kansas & 56,200 \\
\hline 31 & California, Santa Barbara & 68,892 & 88 & Temple & 56,020 \\
\hline 32 & Tennessee & 67,823 & 89 & Rice & 55,450 \\
\hline 33 & Hawaii & 67,391 & 90 & Auburn & 55,290 \\
\hline 34 & McGill & 67,233 & 91 & Boston University & 55,200 \\
\hline 35 & Boston College & 67,184 & 92 & Florida & 55,045 \\
\hline 36 & Southern California & 66,944 & 93 & Texas Tech & 55,036 \\
\hline 37 & SUNY Buffalo & 66,684 & 94 & Vanderbilt & 54,666 \\
\hline 38 & Montreal & 66,631 & 95 & Washington U.-St. Louis & 54,468 \\
\hline 39 & Maryland & 66,419 & 96 & Case Western Reserve & 54,389 \\
\hline 40 & Notre Dame & 65,738 & 97 & Texas A\&M & 54,156 \\
\hline 41 & Michigan & 65,564 & 98 & Oregon & 53,792 \\
\hline 42 & Pennsylvania State & 65,556 & 99 & Kent State & 53,777 \\
\hline 43 & Minnesota & 65,446 & 100 & Oklahoma State & 53,640 \\
\hline 44 & George Washington & 65,328 & 101 & Howard & 53,440 \\
\hline 45 & Colorado State & 65,300 & 102 & Rochester & 52,540 \\
\hline 46 & Miami & 64,935 & 103 & Ohio State & 52,432 \\
\hline 47 & North Carolina State & 64,895 & 104 & Oklahoma & 52,228 \\
\hline 48 & Colorado & 64,388 & 105 & Alabama & 52,205 \\
\hline 49 & Columbia & 64,265 & 106 & Georgia & 51,005 \\
\hline 50 & Western Ontario & 63,872 & 107 & Wayne State & 50,031 \\
\hline 51 & Brown & 63,854 & 108 & South Carolina & 49,838 \\
\hline 52 & North Carolina & 63,750 & 109 & Southern Illinois & 49,117 \\
\hline 53 & Johns Hopkins & 63,702 & 110 & Missouri & 48,854 \\
\hline 54 & Emory & 63,653 & 111 & Ohio University & 48,170 \\
\hline 55 & Cornell & 63,600 & 112 & Louisiana State & 47,715 \\
\hline 56 & Georgetown & 63,538 & 113 & Florida State & 47,000 \\
\hline 57 & Northwestern & 62,847 & & & \\
\hline
\end{tabular}

Salaries of directors are not included in the calculation of medians. Excludes medical and law libraries. See Tables 37 and 44 for statistics related to medical and law library salaries.

Canadian salaries are expressed in US dollars. 


\section{Table 12: Average Professional Salaries in ARL University Libraries RANK ORDER TABLE, FY 2007-2008}

\begin{tabular}{|c|c|c|c|c|c|}
\hline Rank & Institution & Salary & Rank & Institution & Salary \\
\hline 1 & Rutgers & $\$ 82,023$ & 58 & SUNY Buffalo & $\$ 64,414$ \\
\hline 2 & York & 81,659 & 59 & Arizona State & 64,325 \\
\hline 3 & California, Berkeley & 80,749 & 60 & Indiana & 64,257 \\
\hline 4 & New York University & 77,595 & 61 & Pittsburgh & 64,047 \\
\hline 5 & Manitoba & 77,261 & 62 & Illinois, Urbana & 64,009 \\
\hline 6 & Yale & 77,131 & 63 & Washington & 63,655 \\
\hline 7 & Connecticut & 76,946 & 64 & Texas & 63,467 \\
\hline 8 & Toronto & 76,710 & 65 & Iowa & 63,234 \\
\hline 9 & New Mexico & 76,612 & 66 & Pennsylvania & 63,029 \\
\hline 10 & California, Los Angeles & 76,029 & 67 & Virginia Tech & 62,691 \\
\hline 11 & Alberta & 74,787 & 68 & Colorado State & 62,333 \\
\hline 12 & Saskatchewan & 74,526 & 69 & Guelph & 62,069 \\
\hline 13 & Princeton & 74,179 & 70 & Brigham Young & 61,929 \\
\hline 14 & California, San Diego & 74,176 & 71 & Michigan State & 61,743 \\
\hline 15 & California, Davis & 73,853 & 72 & Rice & 61,662 \\
\hline 16 & Harvard & 72,861 & 73 & Nebraska & 61,376 \\
\hline 17 & Queen`s & 72,268 & 74 & Hawaii & 61,330 \\
\hline 18 & Chicago & 72,179 & 75 & Auburn & 61,104 \\
\hline 19 & Dartmouth & 71,642 & 76 & Duke & 60,715 \\
\hline 20 & California, Irvine & 71,572 & 77 & SUNY Albany & 60,304 \\
\hline 21 & Columbia & 70,838 & 78 & Montreal & 60,262 \\
\hline 22 & Southern California & 70,463 & 79 & Texas A\&M & 60,024 \\
\hline 23 & California, Santa Barbara & 70,227 & 80 & Utah & 59,993 \\
\hline 24 & SUNY Stony Brook & 70,027 & 81 & Texas Tech & 59,987 \\
\hline 25 & MIT & 69,719 & 82 & Iowa State & 59,891 \\
\hline 26 & British Columbia & 69,414 & 83 & Wisconsin & 59,685 \\
\hline 27 & Massachusetts & 69,319 & 84 & Laval & 59,630 \\
\hline 28 & California, Riverside & 69,120 & 85 & Kentucky & 59,613 \\
\hline 29 & Delaware & 69,033 & 86 & Kansas & 59,070 \\
\hline 30 & North Carolina State & 68,461 & 87 & Tulane & 59,023 \\
\hline 31 & Waterloo & 68,405 & 88 & Western Ontario & 58,941 \\
\hline 32 & Tennessee & 68,333 & 89 & Illinois, Chicago & 58,925 \\
\hline 33 & Cornell & 68,228 & 90 & Georgia Tech & 58,753 \\
\hline 34 & North Carolina & 67,938 & 91 & Washington State & 58,249 \\
\hline 35 & Boston College & 67,847 & 92 & Alabama & 58,232 \\
\hline 36 & Virginia & 67,602 & 93 & Washington U.-St. Louis & 58,142 \\
\hline 37 & McMaster & 67,549 & 94 & Vanderbilt & 57,775 \\
\hline 38 & Notre Dame & 66,827 & 95 & Missouri & 57,607 \\
\hline 39 & Pennsylvania State & 66,725 & 96 & Case Western Reserve & 57,089 \\
\hline 40 & Georgetown & 66,689 & 97 & Georgia & 57,082 \\
\hline 41 & Michigan & 66,608 & 98 & Ohio State & 56,745 \\
\hline 42 & Minnesota & 66,067 & 99 & Houston & 56,608 \\
\hline 43 & McGill & 66,010 & 100 & Florida & 56,403 \\
\hline 44 & Johns Hopkins & 65,765 & 101 & Kent State & 56,086 \\
\hline 45 & Temple & 65,552 & 102 & Rochester & 55,411 \\
\hline 46 & Colorado & 65,510 & 103 & Oregon & 54,999 \\
\hline 47 & Brown & 65,442 & 104 & Oklahoma State & 54,593 \\
\hline 48 & Purdue & 65,312 & 105 & Southern Illinois & 54,562 \\
\hline 49 & Cincinnati & 65,283 & 106 & Boston University & 54,472 \\
\hline 50 & Northwestern & 65,234 & 107 & Oklahoma & 53,241 \\
\hline 51 & Louisville & 65,230 & 108 & Howard & 53,211 \\
\hline 52 & Miami & 65,171 & 109 & Wayne State & 53,075 \\
\hline 53 & Syracuse & 65,131 & 110 & South Carolina & 51,457 \\
\hline 54 & George Washington & 64,950 & 111 & Ohio University & 48,853 \\
\hline 55 & Maryland & 64,926 & 112 & Louisiana State & 48,711 \\
\hline 56 & Arizona & 64,731 & 113 & Florida State & 48,656 \\
\hline 57 & Emory & 64,551 & & & \\
\hline
\end{tabular}

Reprinted from ARL Annual Salary Survey 2007-2008. Salaries of directors are not included in the calculation of medians. Excludes medical and law libraries. See Tables 38 and 45 for statistics related to medical and law library salaries.

Canadian salaries are expressed in US dollars. 


\section{Table 13: Average Professional Salaries in ARL University Libraries RANK ORdER TABLE, FY 2008-2009}

\begin{tabular}{|c|c|c|c|c|c|}
\hline Rank & Institution & Salary & Rank & Institution & Salary \\
\hline 1 & York & 95,755 & 58 & North Carolina & 67,177 \\
\hline 2 & Toronto & 90,829 & 59 & Purdue & 66,957 \\
\hline 3 & Manitoba & 89,270 & 60 & Cincinnati & 66,847 \\
\hline 4 & Alberta & 89,260 & 61 & Colorado & 66,826 \\
\hline 5 & Rutgers & 88,899 & 62 & Texas & 66,554 \\
\hline 6 & Queen`s & 88,107 & 63 & Pittsburgh & 66,544 \\
\hline 7 & Saskatchewan & 85,401 & 64 & Washington & 66,517 \\
\hline 8 & California, Berkeley & 82,952 & 65 & Arizona & 66,447 \\
\hline 9 & Yale & 82,622 & 66 & Northwestern & 66,328 \\
\hline 10 & British Columbia & 79,910 & 67 & Temple & 66,114 \\
\hline 11 & Connecticut & 79,855 & 68 & Pennsylvania & 65,914 \\
\hline 12 & California, Davis & 79,652 & 69 & Nebraska & 65,751 \\
\hline 13 & New York University & 78,920 & 70 & Virginia Tech & 65,516 \\
\hline 14 & McMaster & 78,776 & 71 & Syracuse & 65,441 \\
\hline 15 & New Mexico & 78,591 & 72 & Brigham Young & 63,925 \\
\hline 16 & Harvard & 78,019 & 73 & Michigan State & 63,867 \\
\hline 17 & Princeton & 77,939 & 74 & Indiana & 63,786 \\
\hline 18 & Waterloo & 77,432 & 75 & Houston & 63,512 \\
\hline 19 & Guelph & 77,319 & 76 & Iowa & 63,196 \\
\hline 20 & California, Irvine & 76,045 & 77 & Arizona State & 63,110 \\
\hline 21 & California, San Diego & 76,016 & 78 & Duke & 63,058 \\
\hline 22 & SUNY Stony Brook & 75,948 & 79 & Tulane & 62,896 \\
\hline 23 & California, Los Angeles & 75,812 & 80 & Iowa State & 61,863 \\
\hline 24 & MIT & 75,733 & 81 & Louisville & 61,851 \\
\hline 25 & Chicago & 74,480 & 82 & Rice & 61,433 \\
\hline 26 & California, Riverside & 74,166 & 83 & Illinois, Chicago & 61,206 \\
\hline 27 & Southern California & 73,788 & 84 & Utah & 61,119 \\
\hline 28 & McGill & 73,148 & 85 & Kansas & 61,056 \\
\hline 29 & Delaware & 72,792 & 86 & Georgia Tech & 60,944 \\
\hline 30 & Dartmouth & 72,539 & 87 & Kentucky & 60,909 \\
\hline 31 & Columbia & 72,352 & 88 & Texas Tech & 60,494 \\
\hline 32 & California, Santa Barbara & 71,466 & 89 & Wisconsin & 60,304 \\
\hline 33 & George Washington & 70,759 & 90 & Auburn & 60,231 \\
\hline 34 & North Carolina State & 70,667 & 91 & Washington State & 59,820 \\
\hline 35 & Massachusetts & 70,508 & 92 & Vanderbilt & 59,810 \\
\hline 36 & Tennessee & 70,344 & 93 & Washington U.-St. Louis & 59,497 \\
\hline 37 & Michigan & 70,031 & 94 & Texas A\&M & 59,250 \\
\hline 38 & SUNY Buffalo & 69,982 & 95 & Boston University & 59,236 \\
\hline 39 & Georgetown & 69,739 & 96 & Florida & 58,974 \\
\hline 40 & Boston College & 69,672 & 97 & Alabama & 58,655 \\
\hline 41 & Cornell & 69,564 & 98 & Case Western Reserve & 57,972 \\
\hline 42 & Pennsylvania State & 69,421 & 99 & Georgia & 57,628 \\
\hline 43 & Montreal & 69,342 & 100 & Ohio State & 57,439 \\
\hline 44 & Virginia & 69,246 & 101 & Kent State & 57,317 \\
\hline 45 & Miami & 69,165 & 102 & Southern Illinois & 57,003 \\
\hline 46 & Emory & 69,025 & 103 & Oregon & 56,965 \\
\hline 47 & Laval & 68,511 & 104 & Oklahoma State & 56,703 \\
\hline 48 & Colorado State & 68,355 & 105 & Rochester & 55,965 \\
\hline 49 & Notre Dame & 68,224 & 106 & Missouri & 55,264 \\
\hline 50 & Western Ontario & 68,168 & 107 & Wayne State & 54,962 \\
\hline 51 & Minnesota & 67,975 & 108 & Oklahoma & 54,228 \\
\hline 52 & Maryland & 67,924 & 109 & Ohio University & 54,210 \\
\hline 53 & Brown & 67,804 & 110 & Howard & 53,741 \\
\hline 54 & Hawaii & 67,491 & 111 & South Carolina & 53,670 \\
\hline 55 & Johns Hopkins & 67,265 & 112 & Louisiana State & 51,839 \\
\hline 56 & SUNY Albany & 67,251 & 113 & Florida State & 49,803 \\
\hline 57 & Illinois, Urbana & 67,213 & & & \\
\hline
\end{tabular}

Salaries of directors are not included in the calculation of medians. Excludes medical and law libraries. See Tables 38 and 45 for statistics related to medical and law library salaries.

Canadian salaries are expressed in US dollars. 


\section{Table 14: Average, Median, and Beginning Professional Salaries IN ARL University LibRARIES \\ SuMmary OF RANKINGS, FY 2005-2006 to 2008-2009}

\begin{tabular}{|c|c|c|c|c|c|c|c|c|c|c|c|c|}
\hline \multirow{2}{*}{$\begin{array}{l}\text { Institution } \\
\end{array}$} & \multicolumn{4}{|c|}{ Average Salaries } & \multicolumn{4}{|c|}{ Median Salaries } & \multicolumn{4}{|c|}{ Beginning Salaries } \\
\hline & 2006 & 2007 & 2008 & 2009 & 2006 & 2007 & 2008 & 2009 & 2006 & 2007 & 2008 & 2009 \\
\hline Alabama & 97 & 98 & 92 & 97 & 100 & 101 & 96 & 105 & 92 & 84 & 74 & 73 \\
\hline Alberta & 28 & 17 & 11 & 4 & 6 & 3 & 2 & 1 & 77 & 43 & 38 & 8 \\
\hline Arizona & 40 & 38 & 56 & 65 & 77 & 55 & 56 & 62 & 22 & 21 & 7 & 26 \\
\hline Arizona State & 84 & 69 & 59 & 77 & 69 & 66 & 41 & 66 & 51 & 23 & 34 & 60 \\
\hline Auburn & 78 & 77 & 75 & 90 & 66 & 52 & 63 & 90 & 15 & 15 & 25 & 50 \\
\hline Boston University & 95 & 104 & 106 & 40 & 98 & 97 & 100 & 91 & 107 & 112 & 62 & 70 \\
\hline Boston College & 31 & 36 & 35 & 95 & 35 & 36 & 31 & 35 & 50 & 58 & 57 & 70 \\
\hline Brigham Young & 54 & 68 & 70 & 72 & 47 & 59 & 55 & 60 & 13 & 17 & 14 & 22 \\
\hline British Columbia & 74 & 41 & 26 & 10 & 67 & 32 & 22 & 9 & 88 & 20 & 15 & 2 \\
\hline Brown & 36 & 53 & 47 & 53 & 37 & 61 & 42 & 51 & 89 & 84 & 94 & 99 \\
\hline California, Berkeley & 1 & 2 & 3 & 8 & 1 & 6 & 5 & 10 & 57 & 64 & 69 & 35 \\
\hline California, Davis & 7 & 18 & 15 & 12 & 3 & 8 & 3 & 8 & 57 & 64 & 69 & 35 \\
\hline California, Irvine & 13 & 8 & 20 & 20 & 3 & 5 & 13 & 16 & 57 & 64 & 69 & 35 \\
\hline California, Los Angeles & 3 & 5 & 10 & 23 & 3 & 7 & 12 & 19 & 57 & 64 & 69 & 35 \\
\hline California, Riverside & 25 & 29 & 28 & 26 & 24 & 22 & 24 & 18 & 25 & 26 & 41 & 27 \\
\hline California, San Diego & 11 & 11 & 14 & 21 & 13 & 10 & 13 & 16 & 57 & 44 & 69 & 35 \\
\hline California, Santa Barbara & 14 & 19 & 23 & 32 & 15 & 21 & 27 & 31 & 57 & 64 & 43 & 35 \\
\hline Case Western Reserve & 87 & 94 & 96 & 98 & 79 & 88 & 90 & 96 & 92 & 104 & 108 & 110 \\
\hline Chicago & 15 & 14 & 18 & 25 & 17 & 18 & 23 & 28 & 10 & 9 & 12 & 21 \\
\hline Cincinnati & 41 & 49 & 49 & 60 & 41 & 53 & 47 & 64 & 92 & 84 & 94 & 100 \\
\hline Colorado & 63 & 71 & 46 & 61 & 50 & 63 & 49 & 48 & 28 & 37 & 58 & 51 \\
\hline Colorado State & 53 & 55 & 68 & 48 & 53 & 41 & 53 & 45 & 44 & 45 & 29 & 42 \\
\hline Columbia & 17 & 12 & 21 & 31 & 31 & 26 & 46 & 49 & 3 & 2 & 1 & 4 \\
\hline Connecticut & 6 & 7 & 7 & 11 & 8 & 15 & 9 & 12 & 2 & 5 & 5 & 22 \\
\hline Cornell & 23 & 28 & 33 & 41 & 43 & 43 & 40 & 55 & 23 & 22 & 16 & 28 \\
\hline Dartmouth & 18 & 20 & 19 & 30 & 16 & 20 & 16 & 30 & 68 & 75 & 74 & 67 \\
\hline Delaware & 19 & 26 & 29 & 29 & 18 & 28 & 34 & 24 & 44 & 64 & 28 & 58 \\
\hline Duke & 68 & 75 & 76 & 78 & 74 & 76 & 81 & 78 & 73 & 93 & 34 & 51 \\
\hline Emory & 46 & 60 & 57 & 46 & 46 & 54 & 51 & 54 & 68 & 84 & 94 & 83 \\
\hline Florida & 86 & 93 & 100 & 96 & 91 & 93 & 98 & 92 & 16 & 27 & 44 & 73 \\
\hline Florida State & 109 & 113 & 113 & 113 & 109 & 113 & 111 & 113 & 51 & 37 & 44 & 73 \\
\hline George Washington & 35 & 40 & 54 & 33 & 34 & 62 & 62 & 44 & 16 & 27 & 44 & 28 \\
\hline Georgetown & 32 & 43 & 40 & 39 & 40 & 51 & 48 & 56 & 16 & 11 & 16 & 43 \\
\hline Georgia & 96 & 99 & 97 & 99 & 101 & 104 & 106 & 106 & 112 & 111 & 108 & 105 \\
\hline Georgia Tech & 77 & 72 & 90 & 86 & 86 & 85 & 86 & 86 & 8 & 17 & 44 & 73 \\
\hline Guelph & 76 & 56 & 69 & 19 & 58 & 56 & 66 & 23 & 103 & 80 & 90 & 1 \\
\hline Harvard & 16 & 15 & 16 & 16 & 22 & 24 & 26 & 25 & 11 & 9 & 8 & 18 \\
\hline Hawaii & 52 & 51 & 74 & 54 & 39 & 40 & 54 & 33 & 74 & 74 & 42 & 65 \\
\hline Houston & 112 & 88 & 99 & 75 & 112 & 89 & 108 & 59 & 68 & 64 & 74 & 73 \\
\hline Howard & 102 & 109 & 108 & 110 & 97 & 108 & 93 & 101 & 91 & 103 & 107 & 108 \\
\hline Illinois, Chicago & 73 & 83 & 89 & 83 & 85 & 86 & 84 & 85 & 16 & 27 & 9 & 28 \\
\hline Illinois, Urbana & 42 & 54 & 62 & 57 & 75 & 71 & 68 & 61 & 16 & 23 & 26 & 41 \\
\hline
\end{tabular}


Table 14: Average, Median, and Beginning Professional Salaries IN ARL UNIVERSITY LIBRARIES

SuMMary OF RANKINGS, FY 2005-2006 to 2008-2009

\begin{tabular}{|c|c|c|c|c|c|c|c|c|c|c|c|c|}
\hline Institution & \multicolumn{4}{|c|}{ Average Salaries } & \multicolumn{4}{|c|}{ Median Salaries } & \multicolumn{4}{|c|}{ Beginning Salaries } \\
\hline FY & 2006 & 2007 & 2008 & 2009 & 2006 & 2007 & 2008 & 2009 & 2006 & 2007 & 2008 & 2009 \\
\hline Indiana & 55 & 65 & 60 & 74 & 54 & 64 & 60 & 70 & 78 & 92 & 74 & 90 \\
\hline Iowa & 66 & 67 & 65 & 76 & 81 & 83 & 79 & 83 & 28 & 45 & 74 & 85 \\
\hline Iowa State & 83 & 76 & 82 & 80 & 71 & 72 & 75 & 74 & 28 & 37 & 39 & 60 \\
\hline Johns Hopkins & 39 & 44 & 44 & 55 & 48 & 46 & 57 & 53 & 12 & 7 & 4 & 9 \\
\hline Kansas & 58 & 86 & 86 & 85 & 65 & 78 & 82 & 87 & 28 & 45 & 74 & 16 \\
\hline Kent State & 105 & 96 & 101 & 101 & 94 & 94 & 92 & 99 & 1 & 1 & 31 & 59 \\
\hline Kentucky & 93 & 91 & 85 & 87 & 83 & 81 & 71 & 76 & 64 & 81 & 91 & 85 \\
\hline Laval & 108 & 97 & 84 & 47 & 73 & 73 & 39 & 29 & 98 & 94 & 68 & 24 \\
\hline Louisiana State & 113 & 112 & 112 & 112 & 113 & 112 & 113 & 112 & 87 & 99 & 94 & 105 \\
\hline Louisville & 45 & 48 & 51 & 81 & 26 & 27 & 43 & 82 & 79 & 99 & 100 & 107 \\
\hline McGill & 79 & 39 & 43 & 28 & 36 & 17 & 32 & 34 & 109 & 59 & 65 & 13 \\
\hline McMaster & 85 & 64 & 37 & 14 & 60 & 38 & 20 & 11 & 110 & 96 & 89 & 49 \\
\hline Manitoba & 24 & 9 & 5 & 3 & 10 & 4 & 4 & 2 & 90 & 71 & 55 & 33 \\
\hline Maryland & 60 & 61 & 55 & 52 & 56 & 50 & 44 & 39 & 28 & 45 & 44 & 73 \\
\hline Massachusetts & 20 & 25 & 27 & 35 & 11 & 11 & 19 & 21 & 66 & 60 & 63 & 72 \\
\hline MIT & 21 & 24 & 25 & 24 & 21 & 29 & 29 & 25 & 8 & 11 & 5 & 6 \\
\hline Miami & 56 & 42 & 52 & 45 & 63 & 65 & 58 & 46 & 51 & 36 & 16 & 43 \\
\hline Michigan & 26 & 35 & 41 & 37 & 45 & 45 & 36 & 41 & 28 & 45 & 58 & 73 \\
\hline Michigan State & 49 & 59 & 71 & 73 & 42 & 47 & 76 & 71 & 14 & 6 & 9 & 28 \\
\hline Minnesota & 29 & 37 & 42 & 51 & 30 & 39 & 35 & 43 & 79 & 75 & 74 & 91 \\
\hline Missouri & 98 & 105 & 95 & 106 & 102 & 102 & 104 & 110 & 111 & 108 & 91 & 91 \\
\hline Montreal & 103 & 87 & 78 & 43 & 90 & 80 & 69 & 38 & 106 & 107 & 104 & 82 \\
\hline Nebraska & 82 & 78 & 73 & 69 & 95 & 87 & 87 & 80 & 26 & 41 & 16 & 10 \\
\hline New Mexico & 12 & 13 & 9 & 15 & 9 & 9 & 8 & 15 & 28 & 45 & 74 & 91 \\
\hline New York & 8 & 10 & 4 & 13 & 23 & 31 & 21 & 27 & 4 & 2 & 2 & 3 \\
\hline North Carolina & 50 & 47 & 34 & 58 & 57 & 44 & 37 & 52 & 28 & 27 & 44 & 51 \\
\hline North Carolina State & 48 & 27 & 30 & 34 & 76 & 49 & 45 & 47 & 5 & 8 & 9 & 16 \\
\hline Northwestern & 37 & 50 & 50 & 66 & 38 & 42 & 50 & 57 & 79 & 84 & 86 & 73 \\
\hline Notre Dame & 34 & 32 & 38 & 49 & 33 & 30 & 28 & 40 & 79 & 83 & 74 & 91 \\
\hline Ohio University & 110 & 111 & 111 & 100 & 111 & 111 & 112 & 111 & 107 & 104 & 105 & 83 \\
\hline Ohio State & 70 & 85 & 98 & 109 & 68 & 79 & 95 & 103 & 28 & 41 & 54 & 60 \\
\hline Oklahoma & 106 & 110 & 107 & 108 & 104 & 110 & 103 & 104 & 28 & 45 & 74 & 73 \\
\hline Oklahoma State & 100 & 101 & 104 & 104 & 103 & 100 & 105 & 100 & 99 & 99 & 105 & 108 \\
\hline Oregon & 90 & 107 & 103 & 103 & 88 & 103 & 102 & 98 & 92 & 104 & 108 & 91 \\
\hline Pennsylvania & 47 & 52 & 66 & 68 & 49 & 48 & 64 & 65 & 28 & 27 & 39 & 67 \\
\hline Pennsylvania State & 30 & 34 & 39 & 42 & 32 & 37 & 38 & 42 & 44 & 45 & 56 & 69 \\
\hline Pittsburgh & 57 & 62 & 61 & 63 & 61 & 69 & 65 & 73 & 113 & 108 & 111 & 111 \\
\hline Princeton & 4 & 16 & 13 & 17 & 7 & 23 & 18 & 22 & 28 & 45 & 16 & 10 \\
\hline Purdue & 44 & 63 & 48 & 59 & 59 & 82 & 72 & 66 & 16 & 23 & 29 & 43 \\
\hline Queen's & 38 & 23 & 17 & 6 & 25 & 14 & 11 & 5 & 96 & 73 & 64 & 13 \\
\hline Rice & 72 & 82 & 72 & 82 & 87 & 92 & 83 & 89 & 97 & 98 & 85 & 103 \\
\hline
\end{tabular}


Table 14: Average, Median, and Beginning Professional Salaries IN ARL UNIVERSITY LIBRARIES

SUMMARY OF RANKINGS, FY 2005-2006 to 2008-2009

\begin{tabular}{|c|c|c|c|c|c|c|c|c|c|c|c|c|}
\hline \multirow{2}{*}{$\begin{array}{l}\text { Institution } \\
\\
\end{array}$} & \multicolumn{4}{|c|}{ Average Salaries } & \multicolumn{4}{|c|}{ Median Salaries } & \multicolumn{4}{|c|}{ Beginning Salaries } \\
\hline & 2006 & 2007 & 2008 & 2009 & 2006 & 2007 & 2008 & 2009 & 2006 & 2007 & 2008 & 2009 \\
\hline Rochester & 101 & 102 & 102 & 105 & 99 & 99 & 101 & 102 & 79 & 95 & 103 & 104 \\
\hline Rutgers & 5 & 1 & 1 & 5 & 2 & 1 & 1 & 3 & 7 & 16 & 13 & 25 \\
\hline Saskatchewan & 65 & 31 & 12 & 7 & 52 & 25 & 10 & 7 & 105 & 102 & 53 & 12 \\
\hline South Carolina & 104 & 108 & 110 & 111 & 108 & 106 & 109 & 108 & 99 & 108 & 111 & 111 \\
\hline Southern California & 2 & 21 & 22 & 27 & 19 & 33 & 33 & 36 & 5 & 11 & 16 & 20 \\
\hline Southern Illinois & 92 & 100 & 105 & 102 & 106 & 107 & 107 & 109 & 44 & 61 & 58 & 60 \\
\hline SUNY Albany & 81 & 80 & 77 & 56 & 64 & 68 & 77 & 75 & 64 & 72 & 94 & 101 \\
\hline SUNY Buffalo & 80 & 81 & 58 & 38 & 82 & 84 & 52 & 37 & 49 & 11 & 16 & 43 \\
\hline SUNY Stony Brook & 22 & 22 & 24 & 22 & 20 & 19 & 25 & 20 & 24 & 27 & 34 & 51 \\
\hline Syracuse & 61 & 58 & 53 & 71 & 78 & 74 & 67 & 63 & 79 & 113 & N/A & 113 \\
\hline Temple & 67 & 46 & 45 & 67 & 84 & 57 & 78 & 88 & 76 & 75 & 86 & 64 \\
\hline Tennessee & 43 & 45 & 32 & 36 & 51 & 34 & 30 & 32 & 28 & 45 & 44 & 51 \\
\hline Texas & 51 & 57 & 64 & 62 & 72 & 67 & 73 & 77 & 28 & 17 & 16 & 43 \\
\hline Texas A\&M & 88 & 92 & 79 & 94 & 93 & 96 & 85 & 97 & 28 & 27 & 27 & 28 \\
\hline Texas Tech & 99 & 106 & 81 & 88 & 105 & 105 & 94 & 93 & 51 & 75 & 16 & 43 \\
\hline Toronto & 27 & 4 & 8 & 2 & 12 & 2 & 6 & 4 & 75 & 34 & 33 & 5 \\
\hline Tulane & 89 & 73 & 87 & 79 & 80 & 60 & 80 & 71 & 99 & 84 & 100 & 91 \\
\hline Utah & 94 & 90 & 80 & 84 & 96 & 91 & 88 & 81 & 51 & 45 & 44 & 51 \\
\hline Vanderbilt & 91 & 89 & 94 & 92 & 92 & 95 & 97 & 94 & 79 & 75 & 86 & 91 \\
\hline Virginia & 33 & 33 & 36 & 44 & 28 & 35 & 58 & 68 & 28 & 35 & 34 & 51 \\
\hline Virginia Tech & 75 & 74 & 67 & 70 & 55 & 70 & 61 & 58 & 51 & 84 & 91 & 85 \\
\hline Washington & 59 & 70 & 63 & 64 & 70 & 75 & 70 & 69 & 48 & 45 & 58 & 66 \\
\hline Washington State & 62 & 79 & 91 & 91 & 44 & 77 & 89 & 84 & 68 & 81 & 94 & 101 \\
\hline Washington U.-St. Louis & 64 & 84 & 93 & 93 & 89 & 90 & 99 & 95 & 68 & 84 & 100 & 91 \\
\hline Waterloo & 69 & 30 & 31 & 18 & 29 & 16 & 17 & 13 & 104 & 63 & 52 & 15 \\
\hline Wayne State & 107 & 103 & 109 & 107 & 110 & 109 & 110 & 107 & 79 & 45 & 67 & 89 \\
\hline Western Ontario & 111 & 95 & 88 & 50 & 107 & 98 & 91 & 50 & 67 & 40 & 65 & 34 \\
\hline Wisconsin & 71 & 66 & 83 & 89 & 62 & 58 & 74 & 79 & 63 & 62 & 84 & 88 \\
\hline Yale & 10 & 3 & 6 & 9 & 27 & 13 & 15 & 14 & 27 & 4 & 3 & 7 \\
\hline York & 9 & 6 & 2 & 1 & 14 & 12 & 7 & 6 & 102 & 97 & 32 & 19 \\
\hline
\end{tabular}

Excludes medical and law libraries. 
Page Intentionally Left Blank.

ARL University Libraries · 39 


\section{Table 15: Distribution of Professional Staff in ARL University Libraries by Salary and Position, FY 2008-2009}

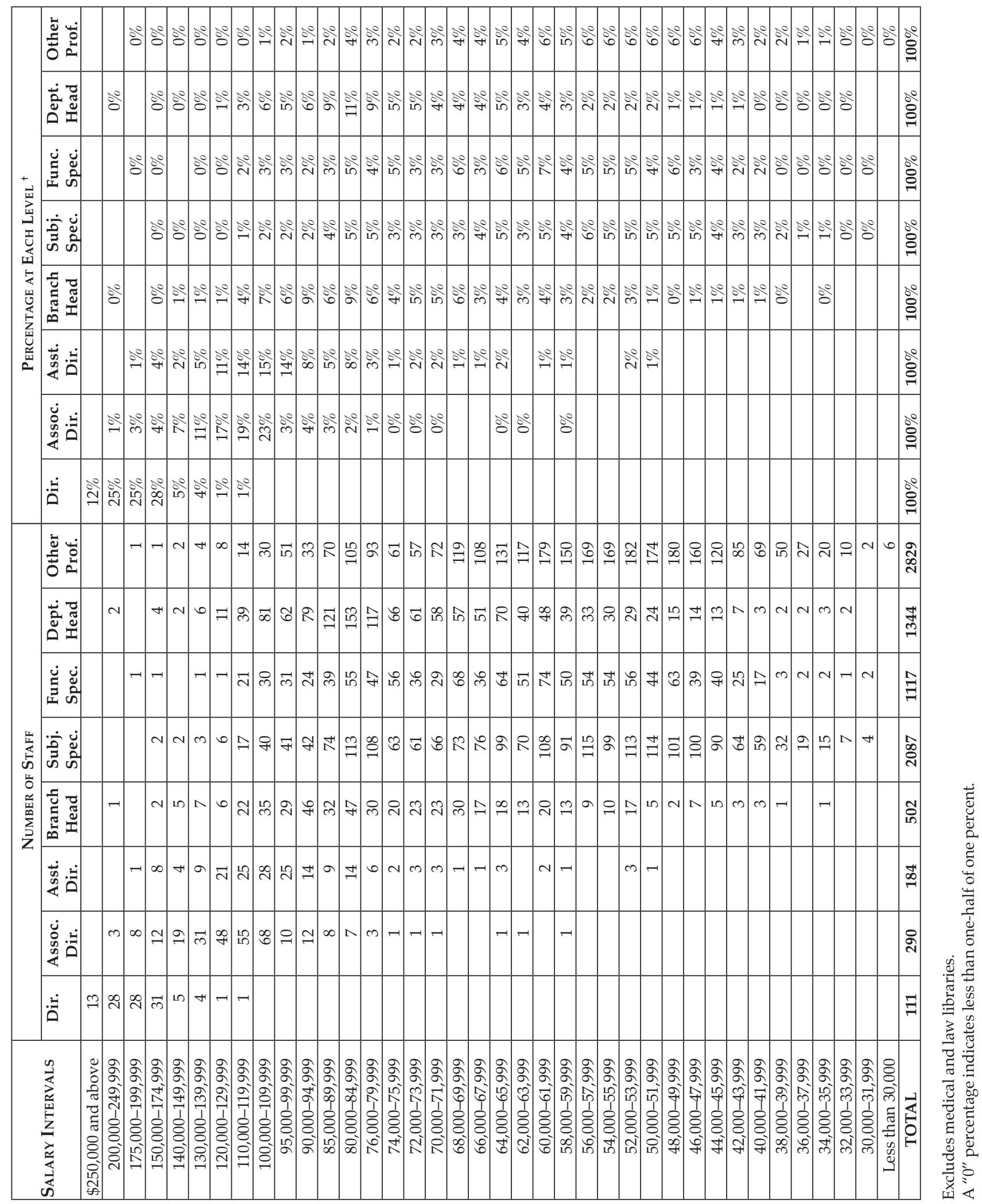




\section{Table 16: Distribution of Professional Staff in ARL University Libraries by Salary, SeX, AND Position, FY 2008-2009}

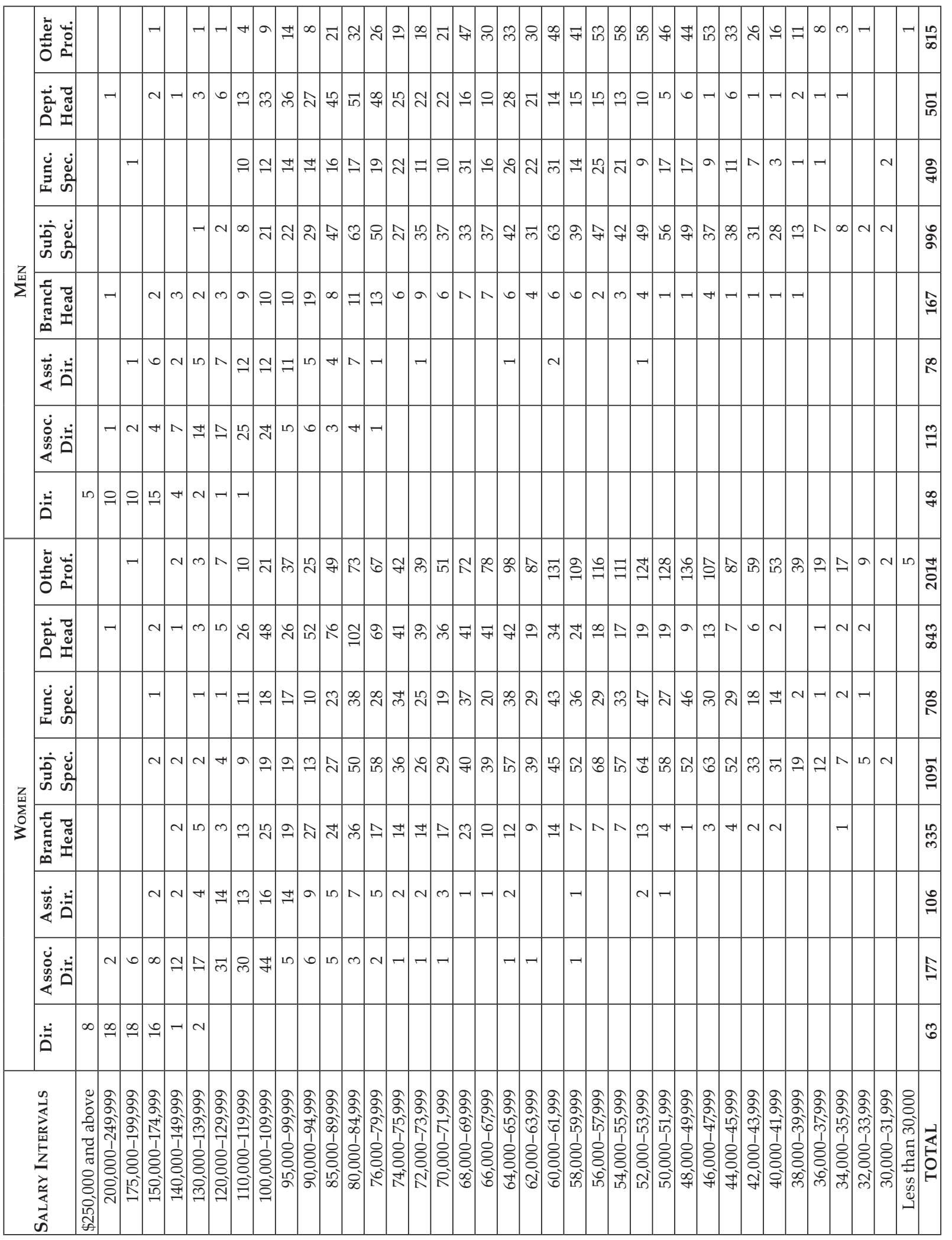




\section{Table 17: Number and Average Salaries of ARL University Librarians by Position AND SeX, FY 2008-2009}

\begin{tabular}{|c|c|c|c|c|c|c|}
\hline \multirow[b]{2}{*}{ Position } & \multicolumn{2}{|c|}{ WOMEN } & \multicolumn{2}{|c|}{ Men } & \multicolumn{2}{|c|}{ TOTAL } \\
\hline & Salary & No. & Salary & No. & Salary & No. \\
\hline Director & $\$ 200,113$ & 63 & $\$ 193,843$ & 48 & $\$ 197,402$ & 111 \\
\hline Associate Director & 118,863 & 177 & 118,200 & 113 & 118,605 & 290 \\
\hline Assistant Director & 100,321 & 106 & 108,492 & 78 & 103,785 & 184 \\
\hline Head, Branch & 79,385 & 335 & 82,917 & 167 & 80,560 & 502 \\
\hline Functional Specialist & 62,284 & 1,091 & 64,422 & 996 & 63,304 & 2,087 \\
\hline Subject Specialist & 64,903 & 708 & 68,194 & 409 & 66,108 & 1,117 \\
\hline \multicolumn{7}{|l|}{ Dept. Head: } \\
\hline Acquisitions & 73,868 & 68 & 73,850 & 30 & 73,863 & 98 \\
\hline Reference & 80,050 & 78 & 80,256 & 29 & 80,106 & 107 \\
\hline Cataloging & 75,260 & 114 & 74,237 & 42 & 74,985 & 156 \\
\hline Serials & 73,653 & 21 & 71,123 & 10 & 72,837 & 31 \\
\hline Documents/Maps & 70,359 & 42 & 70,914 & 23 & 70,555 & 65 \\
\hline Circulation & 73,800 & 62 & 68,428 & 27 & 72,170 & 89 \\
\hline Rare Books/Manuscripts & 81,214 & 42 & 84,607 & 43 & 82,930 & 85 \\
\hline Computer Systems & 91,422 & 23 & 89,486 & 49 & 90,105 & 72 \\
\hline Other & 77,118 & 393 & 79,294 & 248 & 77,960 & 641 \\
\hline \multicolumn{7}{|l|}{ Reference: } \\
\hline Over 14 years experience & 68,561 & 429 & 67,466 & 183 & 68,234 & 612 \\
\hline 10 to 14 years experience & 61,437 & 134 & 60,591 & 55 & 61,191 & 189 \\
\hline 5 to 9 years experience & 56,788 & 216 & 57,433 & 99 & 56,990 & 315 \\
\hline Under 5 years experience & 51,404 & 219 & 49,852 & 67 & 51,041 & 286 \\
\hline \multicolumn{7}{|l|}{ Cataloging: } \\
\hline Over 14 years experience & 65,228 & 278 & 66,970 & 133 & 65,792 & 411 \\
\hline 10 to 14 years experience & 58,692 & 72 & 60,240 & 28 & 59,125 & 100 \\
\hline 5 to 9 years experience & 55,491 & 88 & 55,028 & 48 & 55,327 & 136 \\
\hline Under 5 years experience & 50,888 & 93 & 48,924 & 34 & 50,362 & 127 \\
\hline \multicolumn{7}{|l|}{ Other: } \\
\hline Over 14 years experience & 67,171 & 204 & 71,608 & 64 & 68,230 & 268 \\
\hline 10 to 14 years experience & 60,980 & 64 & 57,314 & 27 & 59,892 & 91 \\
\hline 5 to 9 years experience & 55,672 & 102 & 53,884 & 34 & 55,225 & 136 \\
\hline Under 5 years experience & 48,341 & 115 & 48,606 & 43 & 48,413 & 158 \\
\hline All Positions & $\$ 69,610$ & 5,337 & $\$ 72,344$ & 3,127 & $\$ 70,620$ & 8,464 \\
\hline
\end{tabular}

Canadian salaries are expressed in US dollars. See Table 32 for salaries of Canadian librarians expressed in Canadian dollars. Excludes medical and law libraries. See Tables 39 and 46 for salaries in medical and law libraries. 


\section{Table 18: Number and Average Years of Experience of ARL University Librarians by Position AND SeX, FY 2008-2009}

\begin{tabular}{|c|c|c|c|c|c|c|}
\hline \multirow[b]{2}{*}{ Position } & \multicolumn{2}{|c|}{ WOMEN } & \multicolumn{2}{|c|}{ MeN } & \multicolumn{2}{|c|}{ Total } \\
\hline & Years & No. & Years & No. & Years & No. \\
\hline Director & 32.2 & 63 & 30.9 & 48 & 31.6 & 111 \\
\hline Associate Director & 25.4 & 177 & 23.4 & 113 & 24.6 & 290 \\
\hline Assistant Director & 24.5 & 106 & 23.6 & 78 & 24.1 & 184 \\
\hline Head, Branch & 21.9 & 335 & 22.3 & 167 & 22.1 & 502 \\
\hline Functional Specialist & 13.8 & 1,091 & 12.9 & 996 & 13.3 & 2,087 \\
\hline Subject Specialist & 16.1 & 708 & 17.1 & 409 & 16.4 & 1,117 \\
\hline \multicolumn{7}{|l|}{ Dept. Head: } \\
\hline Acquisitions & 21.1 & 68 & 19.0 & 30 & 20.4 & 98 \\
\hline Reference & 20.4 & 78 & 20.2 & 29 & 20.3 & 107 \\
\hline Cataloging & 22.7 & 114 & 22.0 & 42 & 22.5 & 156 \\
\hline Serials & 20.4 & 21 & 20.6 & 10 & 20.5 & 31 \\
\hline Documents/Maps & 20.8 & 42 & 21.4 & 23 & 21.0 & 65 \\
\hline Circulation & 19.6 & 62 & 16.7 & 27 & 18.7 & 89 \\
\hline Rare Books/Manuscripts & 22.5 & 42 & 24.3 & 43 & 23.4 & 85 \\
\hline Computer Systems & 19.0 & 23 & 18.3 & 49 & 18.5 & 72 \\
\hline Other & 19.8 & 393 & 19.4 & 248 & 19.6 & 641 \\
\hline Public Services & 12.5 & 200 & 11.5 & 71 & 12.3 & 271 \\
\hline Technical Services & 14.7 & 133 & 14.0 & 51 & 14.5 & 184 \\
\hline Administrative Services & 15.8 & 152 & 14.6 & 46 & 15.5 & 198 \\
\hline Reference & 14.6 & 998 & 14.9 & 404 & 14.7 & 1,402 \\
\hline Cataloger & 17.0 & 531 & 17.7 & 243 & 17.2 & 774 \\
\hline All Positions & 17.0 & 5,337 & 16.6 & 3,127 & 16.8 & 8,464 \\
\hline
\end{tabular}

Includes Canadian libraries. See Table 33 for comparable figures in Canadian libraries only.

Excludes medical and law libraries. See Tables 40 and 47 for comparable figures in medical and law libraries. 
Table 19: Number and Average Salaries of ARL University Librarians by YeARS OF EXPERIENCE AND SEX, FY 2008-2009

\begin{tabular}{|c|c|c|c|c|c|c|c|}
\hline \multirow[b]{2}{*}{ Experience } & \multicolumn{2}{|c|}{ WOMEN } & \multicolumn{2}{|c|}{ Men } & \multicolumn{2}{|c|}{ TOTAL } & \multirow{2}{*}{$\begin{array}{c}\% \text { of } \\
\text { TotAL }\end{array}$} \\
\hline & Salary & No. & Salary & No. & Salary & No. & \\
\hline $0-3$ years & $\$ 51,180$ & 712 & $\$ 52,436$ & 352 & $\$ 51,596$ & 1,064 & $13 \%$ \\
\hline $4-7$ years & 55,622 & 676 & 58,046 & 438 & 56,575 & 1,114 & $13 \%$ \\
\hline $8-11$ years & 62,442 & 706 & 63,739 & 476 & 62,964 & 1,182 & $14 \%$ \\
\hline $12-15$ years & 66,893 & 563 & 71,015 & 352 & 68,479 & 915 & $11 \%$ \\
\hline 16 - 19 years & 70,282 & 533 & 73,557 & 316 & 71,501 & 849 & $10 \%$ \\
\hline $20-23$ years & 75,002 & 533 & 80,198 & 293 & 76,845 & 826 & $10 \%$ \\
\hline $24-27$ years & 78,700 & 457 & 81,133 & 252 & 79,565 & 709 & $8 \%$ \\
\hline $28-31$ years & 83,511 & 447 & 90,097 & 267 & 85,974 & 714 & $8 \%$ \\
\hline $32-35$ years & 90,078 & 372 & 93,462 & 218 & 91,328 & 590 & $7 \%$ \\
\hline over 35 years & 93,144 & 338 & 94,381 & 163 & 93,546 & 501 & $6 \%$ \\
\hline All Positions & $\$ 69,610$ & 5,337 & $\$ 72,344$ & 3,127 & $\$ 70,620$ & 8,464 & $100 \%$ \\
\hline
\end{tabular}

Canadian salaries are expressed in US dollars. See Table 34 for salaries in Canadian dollars.

Excludes medical and law libraries. See Tables 41 and 48 for salaries in medical and law libraries. 


\section{Table 20: Average Salaries of ARL University Librarians by Position and YeARs of ExPerience, FY 2008-2009}

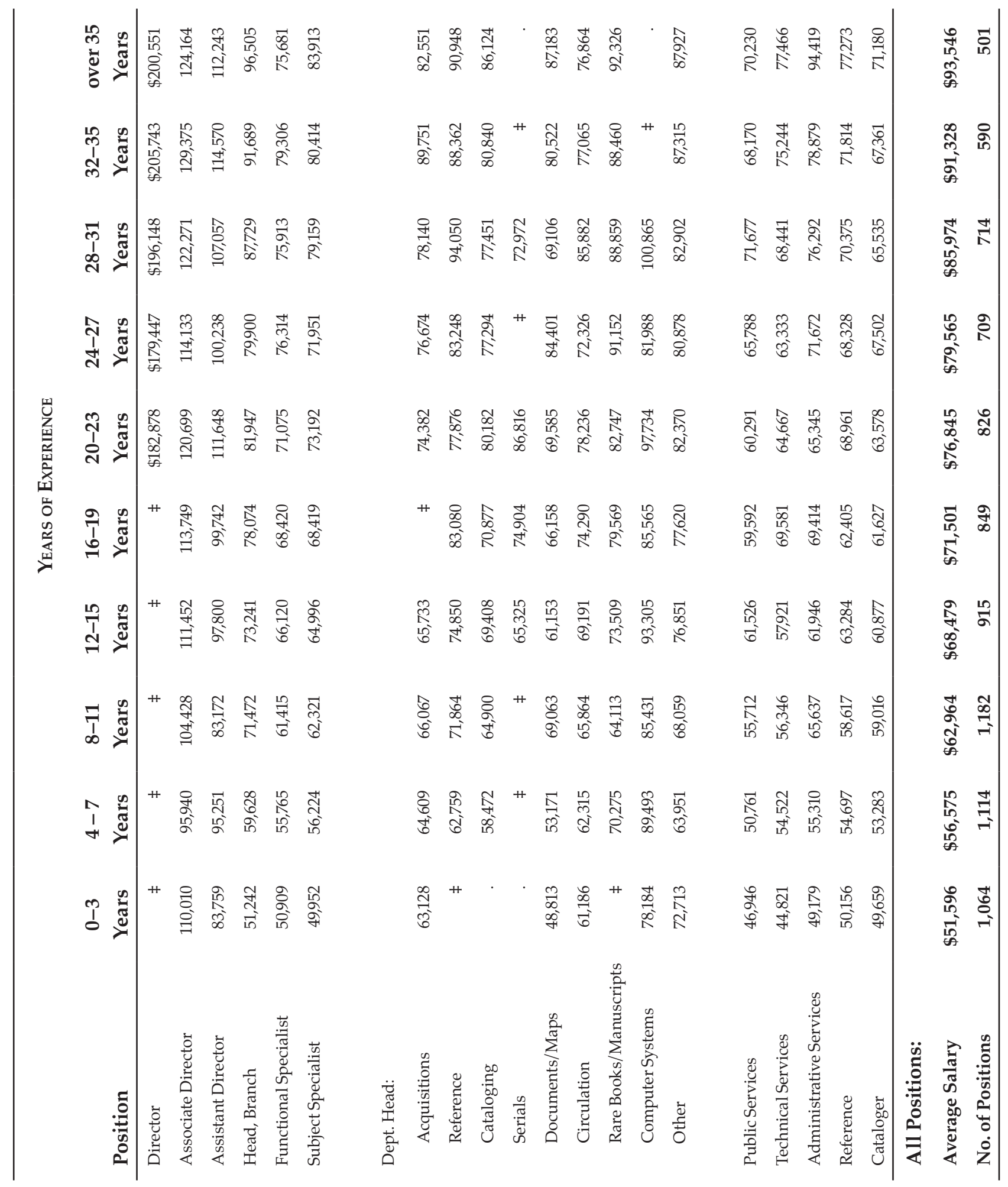

Years of experience reflect total professional experience. Canadian salaries are expressed in US dollars. Excludes medical and law libraries. ₹ Salary data are not published when fewer than four individuals are involved. 


\section{Table 21: Number and Average Salaries of ARL University Librarians by Position AND TyPe Of Institution, FY 2008-2009}

\begin{tabular}{|c|c|c|c|c|c|c|c|c|}
\hline \multirow[b]{2}{*}{ Position } & \multicolumn{2}{|c|}{ CANAdian (14) } & \multicolumn{2}{|c|}{ Private (31) } & \multicolumn{2}{|c|}{ Public (68) } & \multicolumn{2}{|c|}{ Total (113) } \\
\hline & Salary & No. & Salary & No. & Salary & No. & Salary & No. \\
\hline Director & $\$ 166,499$ & 14 & $\$ 232,717$ & 29 & $\$ 188,703$ & 68 & $\$ 197,402$ & 111 \\
\hline Associate Director & 120,119 & 38 & 128,353 & 90 & 112,834 & 162 & 118,605 & 290 \\
\hline Assistant Director & 95,453 & 16 & 107,298 & 72 & 102,540 & 96 & 103,785 & 184 \\
\hline Head, Branch & 98,849 & 59 & 84,247 & 132 & 75,526 & 311 & 80,560 & 502 \\
\hline Functional Specialist & 75,094 & 150 & 66,135 & 805 & 59,729 & 1,132 & 63,304 & 2,087 \\
\hline Subject Specialist & 73,079 & 115 & 66,634 & 384 & 64,484 & 618 & 66,108 & 1,117 \\
\hline \multicolumn{9}{|l|}{ Dept. Head: } \\
\hline Acquisitions & 88,784 & 14 & 74,037 & 27 & 70,115 & 57 & 73,863 & 98 \\
\hline Reference & 88,929 & 13 & 81,345 & 42 & 76,899 & 52 & 80,106 & 107 \\
\hline Cataloging & 92,532 & 7 & 76,270 & 65 & 72,528 & 84 & 74,985 & 156 \\
\hline Serials & $\neq$ & 3 & 70,218 & 11 & 71,121 & 17 & 72,837 & 31 \\
\hline Documents/Maps & 101,169 & 8 & 66,386 & 14 & 66,217 & 43 & 70,555 & 65 \\
\hline Circulation & 79,264 & 13 & 70,904 & 23 & 70,980 & 53 & 72,170 & 89 \\
\hline Rare Books/Manuscripts & 99,153 & 6 & 79,030 & 26 & 83,007 & 53 & 82,930 & 85 \\
\hline Computer Systems & 91,042 & 7 & 93,167 & 27 & 87,756 & 38 & 90,105 & 72 \\
\hline Other & 91,675 & 52 & 79,350 & 211 & 75,298 & 378 & 77,960 & 641 \\
\hline \multicolumn{9}{|l|}{ Reference: } \\
\hline Over 14 years experience & 88,443 & 91 & 65,440 & 158 & 64,383 & 363 & 68,234 & 612 \\
\hline 10 to 14 years experience & 78,139 & 23 & 61,018 & 52 & 57,850 & 114 & 61,191 & 189 \\
\hline 5 to 9 years experience & 67,768 & 65 & 58,794 & 78 & 52,100 & 172 & 56,990 & 315 \\
\hline Under 5 years experience & 61,576 & 68 & 49,711 & 50 & 47,172 & 168 & 51,041 & 286 \\
\hline \multicolumn{9}{|l|}{ Cataloging: } \\
\hline Over 14 years experience & 82,408 & 35 & 65,609 & 172 & 63,095 & 204 & 65,792 & 411 \\
\hline 10 to 14 years experience & 65,292 & 10 & 59,887 & 39 & 57,334 & 51 & 59,125 & 100 \\
\hline 5 to 9 years experience & 71,490 & 8 & 58,627 & 68 & 49,433 & 60 & 55,327 & 136 \\
\hline Under 5 years experience & 62,006 & 7 & 55,176 & 48 & 46,022 & 72 & 50,362 & 127 \\
\hline \multicolumn{9}{|l|}{ Other: } \\
\hline Over 14 years experience & 83,954 & 15 & 68,970 & 88 & 66,407 & 165 & 68,230 & 268 \\
\hline 10 to 14 years experience & 70,025 & 6 & 64,497 & 30 & 56,275 & 55 & 59,892 & 91 \\
\hline 5 to 9 years experience & 63,614 & 12 & 61,035 & 43 & 50,898 & 81 & 55,225 & 136 \\
\hline Under 5 years experience & 65,981 & 9 & 50,659 & 56 & 45,361 & 93 & 48,413 & 158 \\
\hline All Positions & $\$ 82,295$ & 864 & $\$ 72,287$ & 2,840 & $\$ 67,506$ & 4,760 & $\$ 70,620$ & 8,464 \\
\hline
\end{tabular}

Canadian salaries are expressed in US dollars. Tables 31-34 show Canadian salaries in Canadian dollars. Excludes medical and law libraries.

( ) Indicates the number of ARL libraries in each category.

₹ Salary data are not published when fewer than four individuals are involved. 


\section{TAble 22: Years of Experience of ARL University Librarians By Position ANd Type of Institution, FY 2008-2009}

\begin{tabular}{|c|c|c|c|c|c|c|c|c|}
\hline \multirow[b]{2}{*}{ Position } & \multicolumn{2}{|c|}{ CANADIAN (14) } & \multicolumn{2}{|c|}{ Private (31) } & \multicolumn{2}{|c|}{ Public (68) } & \multicolumn{2}{|c|}{ Total (113) } \\
\hline & Years & No. & Years & No. & Years & No. & Years & No. \\
\hline Director & 29.1 & 14 & 31.8 & 29 & 32.1 & 68 & 31.6 & 111 \\
\hline Associate Director & 23.2 & 38 & 24.3 & 90 & 25.1 & 162 & 24.6 & 290 \\
\hline Assistant Director & 21.9 & 16 & 24.8 & 72 & 24.0 & 96 & 24.1 & 184 \\
\hline Head, Branch & 22.9 & 59 & 22.2 & 132 & 21.9 & 311 & 22.1 & 502 \\
\hline Functional Specialist & 15.3 & 150 & 13.0 & 805 & 13.3 & 1,132 & 13.3 & 2,087 \\
\hline Subject Specialist & 15.3 & 115 & 17.1 & 384 & 16.3 & 618 & 16.4 & 1,117 \\
\hline \multicolumn{9}{|l|}{ Dept. Head: } \\
\hline Acquisitions & 20.9 & 14 & 21.4 & 27 & 19.9 & 57 & 20.4 & 98 \\
\hline Reference & 19.2 & 13 & 20.5 & 42 & 20.5 & 52 & 20.3 & 107 \\
\hline Cataloging & 25.4 & 7 & 22.0 & 65 & 22.6 & 84 & 22.5 & 156 \\
\hline Serials & 25.0 & 3 & 21.7 & 11 & 18.9 & 17 & 20.5 & 31 \\
\hline Documents/Maps & 27.8 & 8 & 17.9 & 14 & 20.8 & 43 & 21.0 & 65 \\
\hline Circulation & 18.0 & 13 & 16.4 & 23 & 19.9 & 53 & 18.7 & 89 \\
\hline Rare Books/Manuscripts & 28.2 & 6 & 19.9 & 26 & 24.6 & 53 & 23.4 & 85 \\
\hline Computer Systems & 19.9 & 7 & 18.7 & 27 & 18.2 & 38 & 18.5 & 72 \\
\hline Other & 17.4 & 52 & 19.4 & 211 & 20.0 & 378 & 19.6 & 641 \\
\hline \multicolumn{9}{|l|}{ Reference: } \\
\hline Over 14 years experience & 25.4 & 91 & 25.7 & 158 & 24.8 & 363 & 25.1 & 612 \\
\hline 10 to 14 years experience & 11.9 & 23 & 12.0 & 52 & 12.0 & 113 & 12.0 & 188 \\
\hline 5 to 9 years experience & 6.8 & 65 & 7.3 & 76 & 7.1 & 172 & 7.1 & 313 \\
\hline Under 5 years experience & 2.5 & 68 & 2.5 & 52 & 2.4 & 168 & 2.5 & 288 \\
\hline \multicolumn{9}{|l|}{ Cataloging: } \\
\hline Over 14 years experience & 28.7 & 35 & 26.0 & 172 & 26.7 & 204 & 26.6 & 411 \\
\hline 10 to 14 years experience & 11.8 & 10 & 11.5 & 39 & 12.1 & 51 & 11.8 & 100 \\
\hline 5 to 9 years experience & 6.8 & 8 & 7.0 & 68 & 6.5 & 60 & 6.7 & 136 \\
\hline Under 5 years experience & 2.0 & 7 & 2.5 & 48 & 2.3 & 72 & 2.3 & 127 \\
\hline \multicolumn{9}{|l|}{ Other: } \\
\hline Over 14 years experience & 23.5 & 15 & 24.3 & 88 & 25.3 & 165 & 24.9 & 268 \\
\hline 10 to 14 years experience & 11.7 & 6 & 11.6 & 30 & 11.9 & 55 & 11.7 & 91 \\
\hline 5 to 9 years experience & 6.7 & 12 & 7.0 & 43 & 7.1 & 81 & 7.0 & 136 \\
\hline Under 5 years experience & 2.4 & 9 & 2.5 & 56 & 2.2 & 93 & 2.3 & 158 \\
\hline All Positions & 16.7 & 864 & 16.7 & 2,840 & 16.9 & 4,760 & 16.8 & 8,464 \\
\hline
\end{tabular}

Excludes medical and law libraries.

( ) Indicates the number of ARL libraries in each category. 


\section{Table 23: Number and Average Salaries of ARL University Librarians by Position and Size of Professional Staff, FY 2008-2009}

\begin{tabular}{|c|c|c|c|c|c|c|c|c|}
\hline \multirow[b]{2}{*}{ Position } & \multicolumn{2}{|c|}{$\begin{array}{c}\text { StafF OVer } 110 \\
(\mathbf{1 6})^{+}\end{array}$} & \multicolumn{2}{|c|}{$\begin{array}{c}\text { STAFF } 75-110 \\
(21)\end{array}$} & \multicolumn{2}{|c|}{$\begin{array}{c}\text { STAFF 50-74 } \\
(46)\end{array}$} & \multicolumn{2}{|c|}{$\begin{array}{c}\text { STAFF 22-49 } \\
(30)^{\ddagger}\end{array}$} \\
\hline & Salary & No. & Salary & No. & Salary & No. & Salary & No. \\
\hline Director & $\$ 238,506$ & 16 & $\$ 205,620$ & 21 & $\$ 189,818$ & 44 & $\$ 180,849$ & 30 \\
\hline Associate Director & 137,579 & 60 & 119,141 & 82 & 111,599 & 87 & 109,213 & 61 \\
\hline Assistant Director & 113,779 & 51 & 95,203 & 34 & 102,566 & 75 & 98,519 & 24 \\
\hline Head, Branch & 85,540 & 160 & 84,049 & 95 & 74,610 & 170 & 79,045 & 77 \\
\hline Functional Specialist & 66,396 & 745 & 64,212 & 511 & 59,950 & 590 & 60,030 & 241 \\
\hline Subject Specialist & 69,974 & 352 & 66,925 & 288 & 62,753 & 374 & 62,796 & 103 \\
\hline \multicolumn{9}{|l|}{ Dept. Head: } \\
\hline Acquisitions & 76,337 & 19 & 84,430 & 14 & 68,784 & 39 & 73,982 & 26 \\
\hline Reference & 85,840 & 29 & 78,335 & 13 & 76,090 & 40 & 80,799 & 25 \\
\hline Cataloging & 79,485 & 56 & 77,395 & 26 & 69,028 & 52 & 74,760 & 22 \\
\hline Serials & 75,971 & 10 & 73,018 & 7 & 75,250 & 7 & 65,765 & 7 \\
\hline Documents/Maps & 75,907 & 19 & 72,988 & 10 & 70,447 & 24 & 60,270 & 12 \\
\hline Circulation & 79,398 & 23 & 77,315 & 17 & 66,326 & 36 & 68,843 & 13 \\
\hline Rare Books/Manuscripts & 96,792 & 16 & 84,975 & 14 & 77,273 & 32 & 79,913 & 23 \\
\hline Computer Systems & 103,360 & 7 & 96,256 & 14 & 87,464 & 37 & 84,305 & 14 \\
\hline Other & 82,716 & 167 & 81,579 & 137 & 73,453 & 247 & 75,996 & 90 \\
\hline \multicolumn{9}{|l|}{ Reference: } \\
\hline Over 14 years experience & 69,004 & 143 & 71,300 & 118 & 66,638 & 213 & 67,277 & 138 \\
\hline 10 to 14 years experience & 61,727 & 44 & 61,118 & 34 & 61,144 & 77 & 60,675 & 34 \\
\hline 5 to 9 years experience & 60,513 & 84 & 57,618 & 70 & 54,462 & 105 & 55,663 & 56 \\
\hline Under 5 years experience & 53,690 & 45 & 52,241 & 68 & 50,732 & 110 & 48,391 & 63 \\
\hline \multicolumn{9}{|l|}{ Cataloging: } \\
\hline Over 14 years experience & 69,950 & 161 & 64,059 & 82 & 61,424 & 124 & 66,117 & 44 \\
\hline 10 to 14 years experience & 64,968 & 30 & 57,848 & 25 & 57,858 & 32 & 51,218 & 13 \\
\hline 5 to 9 years experience & 60,788 & 60 & 51,513 & 26 & 51,224 & 36 & 49,559 & 14 \\
\hline Under 5 years experience & 55,425 & 52 & 48,782 & 24 & 47,285 & 36 & 42,724 & 15 \\
\hline \multicolumn{9}{|l|}{ Other: } \\
\hline Over 14 years experience & 71,155 & 88 & 68,949 & 70 & 65,501 & 77 & 65,274 & 33 \\
\hline 10 to 14 years experience & 62,403 & 31 & 59,480 & 24 & 59,362 & 27 & 53,929 & 9 \\
\hline 5 to 9 years experience & 61,058 & 42 & 52,928 & 49 & 53,827 & 32 & 48,478 & 13 \\
\hline Under 5 years experience & 51,352 & 54 & 48,843 & 41 & 46,431 & 50 & 42,475 & 13 \\
\hline All Positions & $\$ 73,135$ & 2,564 & $\$ 71,063$ & 1,914 & $\$ 68,141$ & 2,773 & $\$ 70,274$ & 1,213 \\
\hline
\end{tabular}

Canadian salaries are expressed in US dollars. For average Canadian salaries (expressed in US dollars) refer to Table 21; Tables 31-34 show Canadian salaries in Canadian dollars. Excludes medical and law libraries.

( ) Indicates the number of ARL libraries in each category.

+ In 1995-1996 and earlier, the first column of this table reported staff over 124; in 1996-1998 over 120; in 1998-1999 over 115; and since 1999-2000, over 110.

‡ No ARL library has fewer than 21 professional staff members. 
Table 24: Years of Experience of ARL University Librarians by Position and Size of Professional Staff, FY 2008-2009

\begin{tabular}{|c|c|c|c|c|c|c|c|c|}
\hline \multirow[b]{2}{*}{ Position } & \multicolumn{2}{|c|}{$\begin{array}{c}\text { StAFF OVER } 110 \\
(\mathbf{1 6})^{\dagger}\end{array}$} & \multicolumn{2}{|c|}{$\begin{array}{c}\text { StAFF } 75-110 \\
(21)\end{array}$} & \multicolumn{2}{|c|}{$\begin{array}{c}\text { STAFF 50-74 } \\
(46)\end{array}$} & \multicolumn{2}{|c|}{$\begin{array}{c}\text { STAFF } 24-49 \\
(30)^{\ddagger}\end{array}$} \\
\hline & Years & No. & Years & No. & Years & No. & Years & No. \\
\hline Director & 33.7 & 16 & 28.1 & 21 & 30.9 & 44 & 34.0 & 30 \\
\hline Associate Director & 25.2 & 60 & 23.3 & 82 & 24.9 & 87 & 25.3 & 61 \\
\hline Assistant Director & 24.4 & 51 & 20.8 & 34 & 25.0 & 75 & 25.6 & 24 \\
\hline Head, Branch & 22.8 & 160 & 20.7 & 95 & 21.9 & 170 & 22.4 & 77 \\
\hline Functional Specialist & 12.6 & 745 & 14.0 & 511 & 13.2 & 590 & 14.5 & 241 \\
\hline Subject Specialist & 16.0 & 352 & 16.7 & 288 & 16.7 & 374 & 16.2 & 103 \\
\hline \multicolumn{9}{|l|}{ Dept. Head: } \\
\hline Acquisitions & 22.8 & 19 & 22.1 & 14 & 19.3 & 39 & 19.6 & 26 \\
\hline Reference & 22.7 & 29 & 16.4 & 13 & 21.0 & 40 & 18.5 & 25 \\
\hline Cataloging & 23.3 & 56 & 22.2 & 26 & 22.9 & 52 & 19.7 & 22 \\
\hline Serials & 22.1 & 10 & 15.7 & 7 & 23.6 & 7 & 19.9 & 7 \\
\hline Documents/Maps & 23.6 & 19 & 18.5 & 10 & 21.5 & 24 & 18.1 & 12 \\
\hline Circulation & 19.6 & 23 & 16.1 & 17 & 17.3 & 36 & 24.5 & 13 \\
\hline Rare Books/Manuscripts & 25.9 & 16 & 23.2 & 14 & 23.3 & 32 & 22.0 & 23 \\
\hline Computer Systems & 18.9 & 7 & 19.8 & 14 & 17.4 & 37 & 20.2 & 14 \\
\hline Other & 21.2 & 167 & 19.4 & 137 & 18.4 & 247 & 20.3 & 90 \\
\hline \multicolumn{9}{|l|}{ Reference: } \\
\hline Over 14 years experience & 25.9 & 143 & 25.6 & 118 & 24.8 & 213 & 24.6 & 138 \\
\hline 10 to 14 years experience & 12.1 & 44 & 12.2 & 34 & 12.0 & 77 & 11.5 & 34 \\
\hline 5 to 9 years experience & 7.1 & 84 & 6.8 & 70 & 7.1 & 105 & 7.2 & 56 \\
\hline Under 5 years experience & 2.7 & 45 & 2.4 & 68 & 2.2 & 110 & 2.7 & 63 \\
\hline \multicolumn{9}{|l|}{ Cataloging: } \\
\hline Over 14 years experience & 26.4 & 161 & 26.4 & 82 & 27.1 & 124 & 25.6 & 26.4 \\
\hline 10 to 14 years experience & 11.5 & 30 & 12.2 & 25 & 11.9 & 32 & 11.5 & 11.5 \\
\hline 5 to 9 years experience & 6.9 & 60 & 7.0 & 26 & 6.6 & 36 & 6.1 & 6.9 \\
\hline Under 5 years experience & 2.4 & 52 & 2.4 & 24 & 2.3 & 36 & 2.0 & 2.4 \\
\hline \multicolumn{9}{|l|}{ Other: } \\
\hline Over 14 years experience & 26.1 & 88 & 23.2 & 70 & 25.1 & 77 & 24.5 & 26.1 \\
\hline 10 to 14 years experience & 11.6 & 31 & 12.0 & 24 & 11.7 & 27 & 11.8 & 11.6 \\
\hline 5 to 9 years experience & 6.9 & 42 & 7.3 & 49 & 6.8 & 32 & 7.1 & 6.9 \\
\hline Under 5 years experience & 2.3 & 54 & 2.4 & 41 & 2.1 & 50 & 2.5 & 2.3 \\
\hline All Positions & 16.7 & 2,564 & 16.3 & 1,914 & 16.9 & 2,773 & 17.8 & 1,213 \\
\hline
\end{tabular}

Excludes medical and law libraries.

( ) Indicates the number of ARL libraries in each category.

† In 1995-1996 and earlier, the first column of this table reported staff over 124; in 1996-1998 over 120; in 1998-1999 over 115; and since 1999-2000, over 110.

‡ No ARL library has fewer than 21 professional staff members. 


\section{Table 25: Average Salaries of ARL University Librarians By Position and Geographic Region, FY 2008-2009}

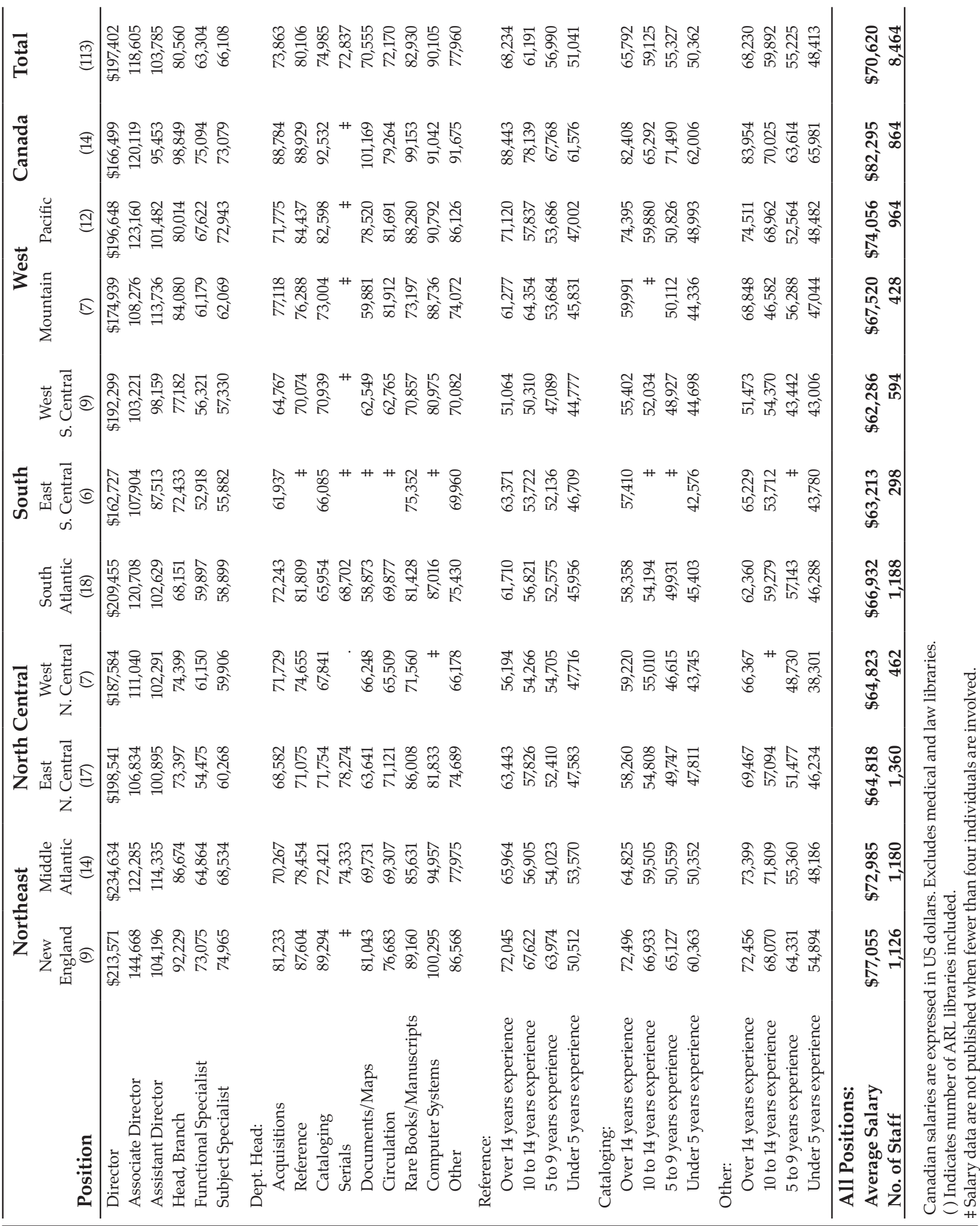




\section{ARL University Libraries by Geographic Region}

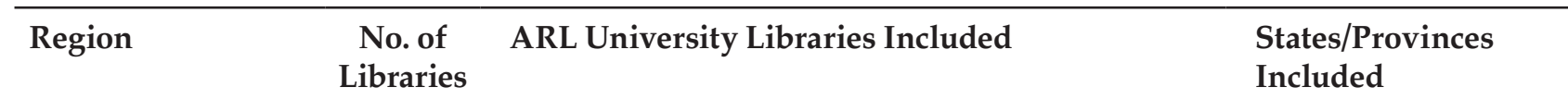

\section{Northeast}

1. New England

2. Middle Atlantic

\section{North Central}

3. East North Central

4. West North Central

\section{South}

5. South Atlantic

6. East South Central

7. West South Central

\section{West}

8. Mountain

9. Pacific

13

Canada

7
Boston University, Boston College, Brown, Connecticut, Dartmouth, Harvard, Massachusetts Institute of Technology, Massachusetts, Yale

14 Columbia; Cornell; New York; Pennsylvania; Pennsylvania State; Pittsburgh; Princeton; Rochester; Rutgers; State University of New York: Albany, Buffalo, Stony Brook; Syracuse; Temple

Case Western Reserve, Chicago, Cincinnati, Illinois-Chicago, Illinois-Urbana, Indiana, Kent State, Michigan, Michigan State, Notre Dame, Northwestern, Ohio University, Ohio State, Purdue, Southern Illinois, Wayne State, Wisconsin

Iowa, Iowa State, Kansas, Minnesota, Missouri, Nebraska, Washington U.-St. Louis

Houston, Louisiana State, Oklahoma, Oklahoma State, Rice, Texas, Texas A\&M, Texas Tech, Tulane

Arizona, Arizona State, Brigham Young, Colorado, Colorado State, New Mexico, Utah

University of California: Berkeley, Davis, Irvine, Los Angeles, Riverside, San Diego, Santa Barbara; Hawaii; Oregon; Southern California; Washington; Washington State

14 Alberta, British Columbia, Guelph, Laval, McGill, McMaster, Manitoba, Montreal, Queen's, Saskatchewan, Toronto, Waterloo, Western Ontario, York
Conn., Mass., Me., N.H., R.I., Vt.

N.J., N.Y., Pa.

Ill., Ind., Mich., Ohio, Wis.

Iowa, Kan., Minn., Mo.,

Neb.,

N. Dak., S. Dak.

Del., DC, Fla., Ga., Md., N.C., S.C., Va., W. Va.

Ala., Ky., Miss., Tenn.

Ark., La., Okla., Tex.

Ariz., Colo., Idaho, Mont., Nev.,

N. Mex., Utah, Wyo.

Alaska, Calif., Hawaii, Ore., Wash.

Alta., B.C., Man., N. Br., Newf., N.S., Ont., P.E.I., Que., Sask. 



\section{US ARL UNIVERSITY LIBRARIES}

TABles 26-30 


\section{Table 26: Average Salaries of US ARL University Librarians by Position and Years of Experience, FY 2008-2009}

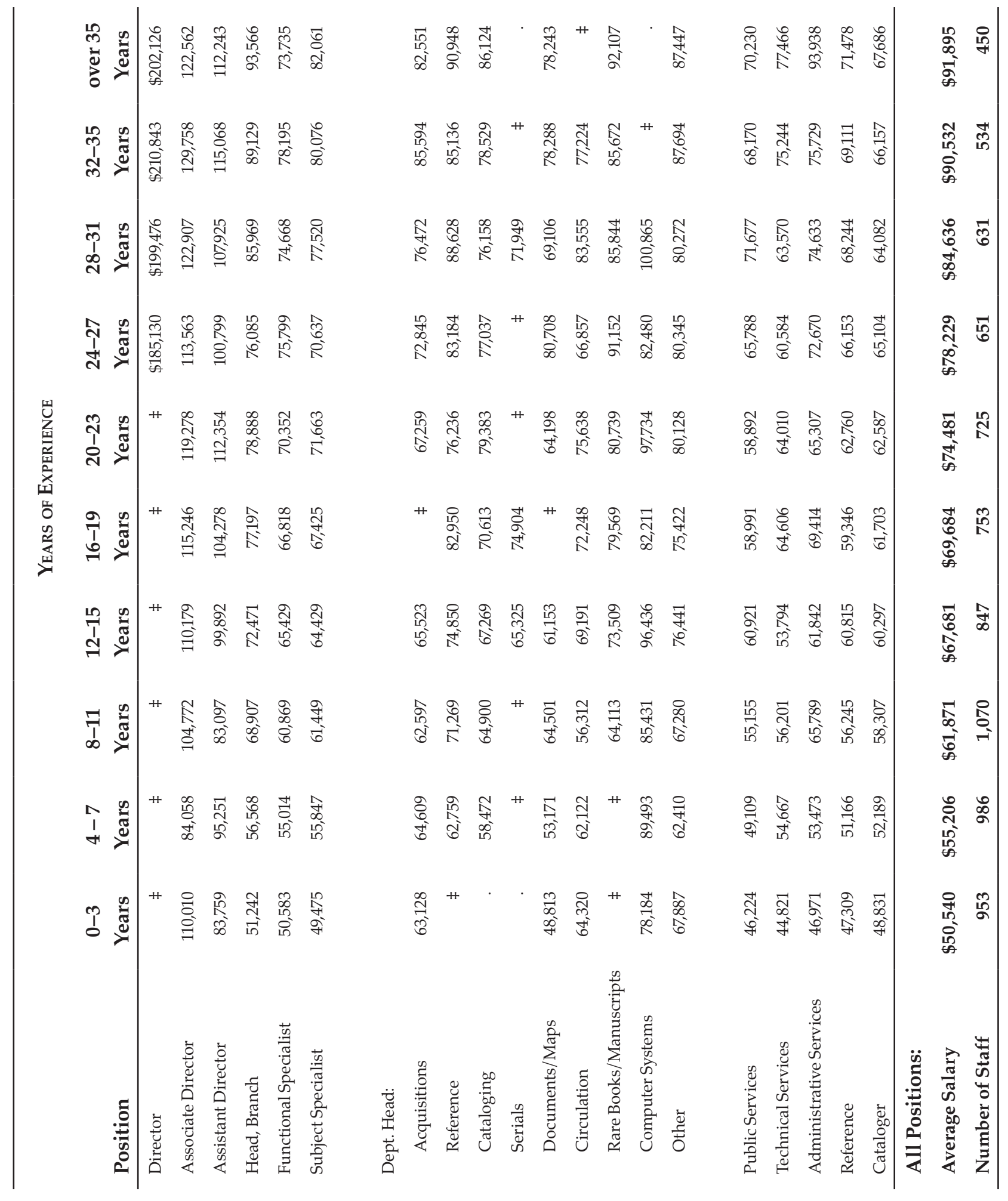




\section{Table 27: Number and Average Salaries of Minority US ARL University Librarians by Position and Sex, FY 2008-2009}

\begin{tabular}{|c|c|c|c|c|c|c|}
\hline \multirow[b]{2}{*}{ Position } & \multicolumn{2}{|c|}{ WOMEN } & \multicolumn{2}{|c|}{ Men } & \multicolumn{2}{|c|}{ Total } \\
\hline & Salary & No. & Salary & No. & Salary & No. \\
\hline Director & $\neq$ & 4 & $\neq$ & 2 & $\$ 178,045$ & 6 \\
\hline Associate Director & $\neq$ & 20 & $\neq$ & 3 & 117,366 & 23 \\
\hline Assistant Director & $\neq$ & 1 & $\neq$ & 5 & 110,640 & 6 \\
\hline Head, Branch & 70,175 & 38 & 84,488 & 12 & 73,610 & 50 \\
\hline Functional Specialist & 59,959 & 152 & 62,325 & 128 & 61,040 & 280 \\
\hline Subject Specialist & 62,373 & 138 & 64,433 & 56 & 62,968 & 194 \\
\hline \multicolumn{7}{|l|}{ Dept. Head: } \\
\hline Acquisitions & $\neq$ & 5 & $\neq$ & 3 & 77,384 & 8 \\
\hline Reference & 82,070 & 5 & . & & 82,070 & 5 \\
\hline Cataloging & $\neq$ & 13 & $\neq$ & 2 & 75,126 & 15 \\
\hline Serials & $\neq$ & 2 & . & & $\neq$ & 2 \\
\hline Documents/Maps & $\neq$ & 3 & $\neq$ & 1 & 64,578 & 4 \\
\hline Circulation & $\neq$ & 7 & $\neq$ & 3 & 71,034 & 10 \\
\hline Rare Books/Manuscripts & $\neq$ & 5 & $\neq$ & 1 & 75,659 & 6 \\
\hline Computer Systems & $\neq$ & 2 & $\neq$ & 9 & 77,999 & 11 \\
\hline Other & 78,617 & 36 & 82,963 & 16 & 79,954 & 52 \\
\hline \multicolumn{7}{|l|}{ Reference: } \\
\hline Over 14 years experience & 65,240 & 55 & 65,949 & 19 & 65,422 & 74 \\
\hline 10 to 14 years experience & 61,018 & 13 & 56,256 & 7 & 59,351 & 20 \\
\hline 5 to 9 years experience & 52,966 & 20 & 60,172 & 8 & 55,025 & 28 \\
\hline Under 5 years experience & 48,352 & 36 & 48,424 & 9 & 48,367 & 45 \\
\hline \multicolumn{7}{|l|}{ Cataloging: } \\
\hline Over 14 years experience & 61,077 & 42 & 70,762 & 12 & 63,229 & 54 \\
\hline 10 to 14 years experience & 60,564 & 17 & 57,435 & 5 & 59,853 & 22 \\
\hline 5 to 9 years experience & 51,680 & 17 & 54,777 & 11 & 52,896 & 28 \\
\hline Under 5 years experience & 50,942 & 20 & 53,132 & 5 & 51,380 & 25 \\
\hline \multicolumn{7}{|l|}{ Other: } \\
\hline Over 14 years experience & 62,676 & 23 & 73,725 & 5 & 64,649 & 28 \\
\hline 10 to 14 years experience & $\neq$ & 11 & $\neq$ & 1 & 58,045 & 12 \\
\hline 5 to 9 years experience & 50,574 & 12 & 44,588 & 4 & 49,078 & 16 \\
\hline Under 5 years experience & $\neq$ & 27 & $\neq$ & 2 & 46,214 & 29 \\
\hline All Positions & $\$ 63,648$ & 724 & $\$ 66,673$ & 329 & $\$ 64,593$ & 1,053 \\
\hline
\end{tabular}

Excludes Canadian libraries. Excludes medical and law libraries.

₹ Salary data are not published when fewer than four individuals are involved in either category. 


\section{Table 28: Number and Average Years of Experience of MiNORITY US ARL University LibRARIANS by Position AND SeX, FY 2008-2009}

\begin{tabular}{|c|c|c|c|c|c|c|}
\hline \multirow[b]{2}{*}{ Position } & \multicolumn{2}{|c|}{ WOMEN } & \multicolumn{2}{|c|}{ Men } & \multicolumn{2}{|c|}{ TOTAL } \\
\hline & Years & No. & Years & No. & Years & No. \\
\hline Director & 28.0 & 4 & 41.0 & 2 & 32.3 & 6 \\
\hline Associate Director & 24.8 & 20 & 32.0 & 3 & 25.7 & 23 \\
\hline Assistant Director & 38.0 & 1 & 11.6 & 5 & 16.0 & 6 \\
\hline Head, Branch & 22.1 & 38 & 18.5 & 12 & 21.2 & 50 \\
\hline Functional Specialist & 13.6 & 152 & 10.9 & 128 & 12.4 & 280 \\
\hline Subject Specialist & 13.9 & 138 & 13.7 & 56 & 13.8 & 194 \\
\hline \multicolumn{7}{|l|}{ Dept. Head: } \\
\hline Acquisitions & 21.8 & 5 & 25.0 & 3 & 23.0 & 8 \\
\hline Reference & 20.4 & 5 & . & & 20.4 & 5 \\
\hline Cataloging & 18.9 & 13 & 23.5 & 2 & 19.5 & 15 \\
\hline Serials & 14.0 & 2 & . & & 14.0 & 2 \\
\hline Documents/Maps & 10.3 & 3 & 11.0 & 1 & 10.5 & 4 \\
\hline Circulation & 18.6 & 7 & 25.3 & 3 & 20.6 & 10 \\
\hline Rare Books/Manuscripts & 14.6 & 5 & 16.0 & 1 & 14.8 & 6 \\
\hline Computer Systems & 22.0 & 2 & 15.6 & 9 & 16.7 & 11 \\
\hline Other & 19.7 & 36 & 20.5 & 16 & 19.9 & 52 \\
\hline Public Services & 7.9 & 33 & 13.8 & 5 & 8.7 & 38 \\
\hline Technical Services & 13.9 & 18 & 8.0 & 1 & 13.6 & 19 \\
\hline Administrative Services & 16.0 & 22 & 12.2 & 6 & 15.1 & 28 \\
\hline Reference & 14.1 & 124 & 14.6 & 43 & 14.2 & 167 \\
\hline Cataloger & 15.1 & 96 & 14.5 & 33 & 14.9 & 129 \\
\hline All Positions & 15.2 & 724 & 13.9 & 329 & 14.8 & 1,053 \\
\hline
\end{tabular}

Includes Canadian libraries. See Table 33 for comparable figures in Canadian libraries only.

Excludes medical and law libraries. See Tables 40 and 47 for comparable figures in medical and law libraries. 
Table 29: Number and Average Salaries OF US ARL UNIVERSITY LIBRARIANS

by YeArs of ExPERIENCE AND SEX, FY 2008-2009

\begin{tabular}{lccccccc}
\hline & \multicolumn{2}{c}{ WoMEN } & \multicolumn{2}{c}{ MEN } & \multicolumn{2}{c}{ Total } & $\%$ OF \\
Experience & Salary & No. & Salary & No. & Salary & No. & TotAL \\
\hline 0-3 years & $\$ 50,180$ & 628 & $\$ 51,235$ & 325 & $\$ 50,540$ & 953 & $13 \%$ \\
$4-7$ years & 53,848 & 588 & 57,213 & 398 & 55,206 & 986 & $13 \%$ \\
$8-11$ years & 61,182 & 633 & 62,870 & 437 & 61,871 & 1,070 & $14 \%$ \\
$12-15$ years & 66,044 & 523 & 70,324 & 324 & 67,681 & 847 & $11 \%$ \\
$16-19$ years & 68,608 & 471 & 71,481 & 282 & 69,684 & 753 & $10 \%$ \\
$20-23$ years & 72,502 & 467 & 78,064 & 258 & 74,481 & 725 & $10 \%$ \\
$24-27$ years & 77,304 & 419 & 79,900 & 232 & 78,229 & 651 & $9 \%$ \\
$28-31$ years & 81,695 & 390 & 89,395 & 241 & 84,636 & 631 & $8 \%$ \\
$32-35$ years & 89,177 & 334 & 92,796 & 200 & 90,532 & 534 & $7 \%$ \\
over 35 years & 91,171 & 301 & 93,358 & 149 & 91,895 & 450 & $6 \%$ \\
\hline All Positions & $\mathbf{6 8 , 1 7 3}$ & $\mathbf{4 7 5 4}$ & $\mathbf{7 1 , 1 6 3}$ & $\mathbf{2 8 4 6}$ & $\mathbf{6 9 , 2 9 3}$ & $\mathbf{7 6 0 0}$ & $\mathbf{1 0 0 \%}$ \\
\hline
\end{tabular}

Excludes Canadian libraries. Excludes medical and law libraries.

Table 30: Number and Average Salaries of Minority US ARL University LibRaRians by Years of Experience AND Sex, FY 2008-2009

\begin{tabular}{lcrrrrrr}
\hline & \multicolumn{2}{c}{ WoMEN } & \multicolumn{2}{c}{ MEN } & \multicolumn{2}{c}{ Total } & $\%$ OF \\
Experience & Salary & No. & Salary & No. & Salary & No. & TotAL \\
\hline $0-3$ years & $\$ 49,170$ & 117 & $\$ 52,712$ & 56 & $\$ 50,316$ & 173 & $16 \%$ \\
$4-7$ years & 52,912 & 115 & 57,399 & 51 & 54,291 & 166 & $16 \%$ \\
$8-11$ years & 58,921 & 84 & 63,981 & 68 & 61,185 & 152 & $14 \%$ \\
$12-15$ years & 66,267 & 86 & 69,070 & 40 & 67,157 & 126 & $12 \%$ \\
$16-19$ years & 69,710 & 79 & 74,054 & 32 & 70,963 & 111 & $11 \%$ \\
$20-23$ years & 70,127 & 83 & 74,761 & 19 & 70,990 & 102 & $10 \%$ \\
$24-27$ years & 62,275 & 42 & 74,255 & 18 & 65,869 & 60 & $6 \%$ \\
$28-31$ years & 73,181 & 42 & 79,867 & 15 & 74,940 & 57 & $5 \%$ \\
$32-35$ years & 93,449 & 39 & 74,219 & 8 & 90,176 & 47 & $4 \%$ \\
over 35 years & 79,297 & 37 & 92,005 & 22 & 84,036 & 59 & $6 \%$ \\
\hline All Positions & $\$ 63,648$ & 724 & $\$ 66,673$ & 329 & $\$ \mathbf{6 6 4 , 5 9 3}$ & $\mathbf{1 , 0 5 3}$ & $\mathbf{1 0 0} \%$ \\
\hline
\end{tabular}

Excludes Canadian libraries. Excludes medical and law libraries. 

Canadian ARL University Libraries

TABLES 31-34 
Table 31: Filled Positions; Average, Median, and Beginning Professional Salaries; and Average Years of Professional Experience in Canadian ARL University Libraries, FY 2008-2009

\begin{tabular}{|c|c|c|c|c|c|c|c|c|}
\hline \multirow[b]{2}{*}{ Institution } & \multirow{2}{*}{$\begin{array}{c}\text { Filled } \\
\text { Positions } \\
\text { FY } 2009 \\
\end{array}$} & \multicolumn{2}{|c|}{ Average Salaries } & \multicolumn{2}{|c|}{ Median Salaries } & \multicolumn{2}{|c|}{ Beginning Salaries } & \multirow{2}{*}{$\begin{array}{l}\text { Average } \\
\text { Yrs. Exp. } \\
\text { FY } 2009 \\
\end{array}$} \\
\hline & & FY 2008 & FY 2009 & FY 2008 & FY 2009 & FY 2008 & FY 2009 & \\
\hline Alberta $^{\ddagger}$ & 67 & $\$ 84,681$ & $\$ 90,161$ & $\$ 93,456$ & $\$ 96,402$ & $\$ 48,685$ & $\$ 96,402$ & 17.4 \\
\hline British Columbia ${ }^{\ddagger}$ & 89 & 78,598 & 80,717 & 77,831 & 81,585 & 51,841 & 81,585 & 14.6 \\
\hline Guelph ${ }^{\ddagger}$ & 47 & 70,281 & 78,100 & 65,980 & 72,654 & 43,695 & 72,654 & 17.8 \\
\hline Laval & 64 & 67,519 & 69,203 & 71,012 & 70,607 & 45,320 & 70,607 & 15.3 \\
\hline McGill ₹ & 64 & 74,744 & 73,887 & 73,291 & 67,912 & 46,000 & 67,912 & 16.9 \\
\hline McMaster $^{\ddagger}$ & 32 & 76,486 & 79,572 & 78,325 & 79,654 & 43,704 & 79,654 & 17.7 \\
\hline Manitoba ${ }^{\ddagger}$ & 42 & 87,483 & 90,172 & 89,632 & 94,294 & 46,799 & 94,294 & 21.9 \\
\hline Montreal ${ }^{\ddagger}$ & 90 & 68,235 & 70,042 & 65,918 & 67,304 & 40,926 & 67,304 & 15.4 \\
\hline Queen's $^{\ddagger}$ & 34 & 81,829 & 88,997 & 83,878 & 90,735 & 46,079 & 90,735 & 19.6 \\
\hline Saskatchewan ${ }^{\ddagger}$ & 39 & 84,386 & 86,263 & 84,307 & 86,845 & 47,101 & 86,845 & 18.0 \\
\hline Toronto $^{\ddagger}$ & 144 & 86,859 & 91,747 & 88,821 & 92,700 & 48,700 & 92,700 & 17.0 \\
\hline Waterloo $^{\ddagger}$ & 34 & 77,455 & 78,214 & 79,343 & 79,102 & 47,198 & 79,102 & 18.4 \\
\hline Western Ontario $^{\ddagger}$ & 63 & 66,739 & 68,857 & 61,423 & 64,517 & 46,000 & 64,517 & 13.4 \\
\hline York ${ }^{\ddagger}$ & 55 & 92,462 & 96,722 & 86,615 & 89,352 & 43,275 & 89,352 & 16.7 \\
\hline
\end{tabular}




\section{Table 32: Number and Average Salaries of Canadian ARL University Librarians by Position And Sex, FY 2008-2009}

\begin{tabular}{|c|c|c|c|c|c|c|}
\hline \multirow[b]{2}{*}{ Position } & \multicolumn{2}{|c|}{ WOMEN } & \multicolumn{2}{|c|}{ MEN } & \multicolumn{2}{|c|}{ Total } \\
\hline & Salary & No. & Salary & No. & Salary & No. \\
\hline Director & $\$ 168,900$ & 8 & $\$ 167,221$ & 6 & $\$ 168,180$ & 14 \\
\hline Associate Director & 120,795 & 24 & 122,252 & 14 & 121,332 & 38 \\
\hline Assistant Director & 92,968 & 8 & 99,866 & 8 & 96,417 & 16 \\
\hline Head, Branch & 100,024 & 45 & 99,278 & 14 & 99,847 & 59 \\
\hline Functional Specialist & 74,623 & 69 & 76,899 & 81 & 75,852 & 150 \\
\hline Subject Specialist & 71,990 & 83 & 78,553 & 32 & 73,817 & 115 \\
\hline \multicolumn{7}{|l|}{ Dept. Head: } \\
\hline Acquisitions & 86,020 & 8 & 94,563 & 6 & 89,681 & 14 \\
\hline Reference & $\neq$ & 12 & $\neq$ & 1 & 89,827 & 13 \\
\hline Cataloging & 93,467 & 7 & . & & 93,467 & 7 \\
\hline Serials & $\neq$ & 3 & . & & $\neq$ & 3 \\
\hline Documents/Maps & $\neq$ & 6 & $\neq$ & 2 & 102,191 & 8 \\
\hline Circulation & $\neq$ & 10 & $\neq$ & 3 & 80,065 & 13 \\
\hline Rare Books/Manuscripts & $\neq$ & 3 & $\neq$ & 3 & 100,154 & 6 \\
\hline Computer Systems & $\neq$ & 2 & $\neq$ & 5 & 91,961 & 7 \\
\hline Other & 92,848 & 36 & 92,046 & 16 & 92,601 & 52 \\
\hline \multicolumn{7}{|l|}{ Reference: } \\
\hline Over 14 years experience & 88,118 & 69 & 93,157 & 22 & 89,336 & 91 \\
\hline 10 to 14 years experience & 78,796 & 15 & 79,178 & 8 & 78,929 & 23 \\
\hline 5 to 9 years experience & 69,060 & 44 & 67,178 & 21 & 68,452 & 65 \\
\hline Under 5 years experience & 61,842 & 57 & 64,043 & 11 & 62,198 & 68 \\
\hline \multicolumn{7}{|l|}{ Cataloging: } \\
\hline Over 14 years experience & 84,688 & 22 & 80,790 & 13 & 83,240 & 35 \\
\hline 10 to 14 years experience & $\neq$ & 8 & $\neq$ & 2 & 65,952 & 10 \\
\hline 5 to 9 years experience & $\neq$ & 7 & $\neq$ & 1 & 72,212 & 8 \\
\hline Under 5 years experience & $\neq$ & 6 & $\neq$ & 1 & 62,633 & 7 \\
\hline \multicolumn{7}{|l|}{ Other: } \\
\hline Over 14 years experience & 82,570 & 9 & 88,150 & 6 & 84,802 & 15 \\
\hline 10 to 14 years experience & $\neq$ & 4 & ‡ & 2 & 70,732 & 6 \\
\hline 5 to 9 years experience & $\neq$ & 11 & $\neq$ & 1 & 64,257 & 12 \\
\hline Under 5 years experience & $\neq$ & 7 & $\neq$ & 2 & 66,647 & 9 \\
\hline All Positions & $\$ 82,146$ & 583 & $\$ 85,159$ & 281 & $\$ 83,126$ & 864 \\
\hline
\end{tabular}

Excludes Canadian medical and law libraries. See Tables 39 and 46 for salaries in medical and law libraries.

Salaries are expressed in Canadian dollars.

+ Salary data are not published when fewer than four individuals are involved in either category. 


\section{Table 33: Number and Average Years of Experience \\ OF CANADIAN ARL UNIVERSITY LIBRARIANS \\ by Position and Sex, FY 2008-2009}

\begin{tabular}{|c|c|c|c|c|c|c|}
\hline \multirow[b]{2}{*}{ Position } & \multicolumn{2}{|c|}{ WoMen } & \multicolumn{2}{|c|}{ MeN } & \multicolumn{2}{|c|}{ TотAL } \\
\hline & Years & No. & Years & No. & Years & No. \\
\hline Director & 32.0 & 8 & 25.2 & 6 & 29.1 & 14 \\
\hline Associate Director & 24.1 & 24 & 21.6 & 14 & 23.2 & 38 \\
\hline Assistant Director & 20.3 & 8 & 23.5 & 8 & 21.9 & 16 \\
\hline Head, Branch & 22.8 & 45 & 23.4 & 14 & 22.9 & 59 \\
\hline Functional Specialist & 15.4 & 69 & 15.2 & 81 & 15.3 & 150 \\
\hline Subject Specialist & 15.0 & 83 & 16.2 & 32 & 15.3 & 115 \\
\hline \multicolumn{7}{|l|}{ Dept. Head: } \\
\hline Acquisitions & 21.4 & 8 & 20.3 & 6 & 20.9 & 14 \\
\hline Reference & 18.3 & 12 & 30.0 & 1 & 19.2 & 13 \\
\hline Cataloging & 25.4 & 7 & . & & 25.4 & 7 \\
\hline Serials & 25.0 & 3 & . & & 25.0 & 3 \\
\hline Documents/Maps & 31.8 & 6 & 15.5 & 2 & 27.8 & 8 \\
\hline Circulation & 20.4 & 10 & 10.0 & 3 & 18.0 & 13 \\
\hline Rare Books/Manuscripts & 29.0 & 3 & 27.3 & 3 & 28.2 & 6 \\
\hline Computer Systems & 22.5 & 2 & 18.8 & 5 & 19.9 & 7 \\
\hline Other & 18.4 & 36 & 15.1 & 16 & 17.4 & 52 \\
\hline Public Services & 6.1 & 8 & 10.3 & 4 & 7.5 & 12 \\
\hline Technical Services & 12.4 & 8 & 18.6 & 5 & 14.8 & 13 \\
\hline Administrations & 15.2 & 15 & 7.5 & 2 & 14.3 & 17 \\
\hline Reference & 12.8 & 185 & 13.5 & 62 & 13.0 & 247 \\
\hline Cataloger & 18.1 & 43 & 24.3 & 17 & 19.9 & 60 \\
\hline All Positions & 16.6 & 583 & 16.9 & 281 & 16.7 & 864 \\
\hline
\end{tabular}

Excludes Canadian medical and law libraries. See Tables 40 and 47 for figures in medical and law libraries. 
Table 34: Number and Average Salaries

of CANADIAN ARL UNIVERSITY LibraRians

by Years of Experience and SeX, FY 2008-2009

\begin{tabular}{lccccccc}
\hline & \multicolumn{2}{c}{ WoMEN } & \multicolumn{2}{c}{ MEN } & \multicolumn{2}{c}{ TotAL } & OF \\
Experience & Salary & No. & Salary & No. & Salary & No. & TotAL \\
\hline $0-3$ years & $\$ 59,248$ & 84 & $\$ 67,563$ & 27 & $\$ 61,271$ & 111 & $\$ 59,248$ \\
$4-7$ years & 68,159 & 88 & 67,002 & 40 & 67,798 & 128 & 68,159 \\
$8-11$ years & 74,104 & 73 & 74,217 & 39 & 74,143 & 112 & 74,104 \\
$12-15$ years & 78,778 & 40 & 79,811 & 28 & 79,203 & 68 & 78,778 \\
$16-19$ years & 83,838 & 62 & 91,693 & 34 & 86,620 & 96 & 83,838 \\
$20-23$ years & 93,625 & 66 & 96,898 & 35 & 94,759 & 101 & 93,625 \\
$24-27$ years & 95,048 & 38 & 96,391 & 20 & 95,511 & 58 & 95,048 \\
$28-31$ years & 96,910 & 57 & 97,577 & 26 & 97,119 & 83 & 96,910 \\
$32-35$ years & 98,994 & 38 & 101,879 & 18 & 99,922 & 56 & 98,994 \\
\hline over 35 years & 110,295 & 37 & 106,330 & 14 & 109,207 & 51 & 110,295 \\
\hline All Positions & $\$ \mathbf{8 2 , 1 4 6}$ & $\mathbf{5 8 3}$ & $\mathbf{\$ 8 5 , 1 5 9}$ & $\mathbf{2 8 1}$ & $\mathbf{\$ 8 3 , 1 2 6}$ & $\mathbf{8 6 4}$ & $\mathbf{\$ 8 2 , 1 4 6}$ \\
\hline
\end{tabular}

Excludes Canadian medical and law libraries. See Tables 41 and 48 for salaries in medical and law libraries.

Salaries are expressed in Canadian dollars 

ARL University Medical Libraries

TABles 35-41 


\section{Table 35: Filled Positions; Average, Median, Beginning Professional Salaries; and Average Years of Professional Experience \\ in ARL University Medical Libraries, FY 2008-2009}

\begin{tabular}{|c|c|c|c|c|c|}
\hline Institution & $\begin{array}{c}\text { Filled } \\
\text { Positions }\end{array}$ & $\begin{array}{c}\text { Average } \\
\text { Salary }\end{array}$ & $\begin{array}{c}\text { Median } \\
\text { Salary }\end{array}$ & $\begin{array}{c}\text { Beginning } \\
\text { Salary }\end{array}$ & $\begin{array}{l}\text { Average } \\
\text { Yrs. Exp. }\end{array}$ \\
\hline Alabama & 3 & $\neq$ & $\neq$ & 35,000 & 10.7 \\
\hline Alberta & 5 & 96,699 & 97,258 & 50,126 & 20.2 \\
\hline Arizona & 16 & 64,038 & 58,706 & 47,093 & 18.6 \\
\hline Boston University & 11 & 56,280 & 50,000 & 42,000 & 10.9 \\
\hline British Columbia & 12 & 77,805 & 81,529 & 53,021 & 17.7 \\
\hline California, Davis & 8 & 80,311 & 60,408 & 46,164 & 25.1 \\
\hline California, Irvine & 1 & ‡ & ‡ & 46,164 & 31.0 \\
\hline California, Los Angeles & 16 & 71,305 & 68,892 & 46,164 & 14.4 \\
\hline California, San Diego & 11 & 66,305 & 66,771 & 46,164 & 14.5 \\
\hline Case Western Reserve & 7 & 62,532 & 66,693 & 35,000 & 26.0 \\
\hline Cincinnati & 18 & 59,134 & 58,431 & 39,000 & 21.1 \\
\hline Columbia & 11 & 61,564 & 61,116 & 51,500 & 15.9 \\
\hline Connecticut & 14 & 78,122 & 74,268 & 55,347 & 17.4 \\
\hline Cornell & 9 & 78,010 & 78,320 & 51,500 & 21.6 \\
\hline Dartmouth & 9 & 61,441 & 60,328 & 42,500 & 19.2 \\
\hline Duke & 18 & 60,297 & 55,743 & 44,000 & 12.5 \\
\hline Emory & 13 & 63,447 & 57,882 & 41,500 & 20.7 \\
\hline Florida & 14 & 58,902 & 55,270 & 42,000 & 16.6 \\
\hline Florida State & 2 & $\neq$ & ‡ & 38,000 & 21.0 \\
\hline George Washington & 13 & 59,940 & 60,803 & 48,000 & 15.4 \\
\hline Georgetown & 11 & 58,700 & 59,250 & 44,000 & 12.5 \\
\hline Harvard & 35 & 75,515 & 73,276 & 48,800 & 10.0 \\
\hline Hawaii & 5 & 46,607 & 45,523 & 42,649 & 17.2 \\
\hline Howard & 5 & 53,754 & 52,009 & 45,000 & 24.6 \\
\hline Illinois, Chicago & 20 & 58,928 & 58,169 & 47,000 & 13.4 \\
\hline Iowa & 10 & 59,648 & 54,540 & 41,000 & 15.7 \\
\hline Johns Hopkins & 29 & 66,744 & 60,626 & 44,000 & 15.7 \\
\hline Kansas & 8 & 52,333 & 49,257 & 38,000 & 14.9 \\
\hline Kentucky & 13 & 57,770 & 57,641 & 41,000 & 21.6 \\
\hline Louisville & 9 & 57,017 & 55,593 & 36,000 & 22.6 \\
\hline McGill & 9 & 72,309 & 65,529 & 37,000 & 17.7 \\
\hline McMaster & 8 & 62,727 & 66,728 & 49,500 & 11.5 \\
\hline Manitoba & 17 & 72,322 & 67,995 & 46,970 & 16.0 \\
\hline Miami & 10 & 71,829 & 72,165 & 44,000 & 19.4 \\
\hline Michigan & 16 & 58,250 & 54,842 & 42,000 & 15.5 \\
\hline Minnesota & 12 & 63,281 & 62,692 & 40,000 & 16.3 \\
\hline Missouri & 10 & 45,421 & 43,000 & 40,000 & 16.8 \\
\hline Montreal & 7 & 72,161 & 71,761 & 41,738 & 18.6 \\
\hline Nebraska & 14 & 62,327 & 56,644 & 45,000 & 18.9 \\
\hline
\end{tabular}

66 · ARL Annual Salary Survey 2008-2009 


\section{Table 35: Filled Positions; Average, Median, Beginning Professional Salaries; and Average Years of Professional Experience in ARL University Medical Libraries, FY 2008-2009}

\begin{tabular}{|c|c|c|c|c|c|}
\hline Institution & $\begin{array}{c}\text { Filled } \\
\text { Positions }\end{array}$ & $\begin{array}{c}\text { Average } \\
\text { Salary }\end{array}$ & $\begin{array}{c}\text { Median } \\
\text { Salary }\end{array}$ & $\begin{array}{l}\text { Beginning } \\
\text { Salary }\end{array}$ & $\begin{array}{l}\text { Average } \\
\text { Yrs. Exp. }\end{array}$ \\
\hline New Mexico & 22 & 70,363 & 67,386 & 39,372 & 17.9 \\
\hline New York University & 30 & 66,047 & 60,309 & 50,000 & 12.2 \\
\hline North Carolina & 32 & 65,912 & 64,002 & 45,000 & 17.9 \\
\hline Northwestern & 17 & 61,237 & 61,427 & 41,000 & 15.9 \\
\hline Ohio State & 9 & 66,036 & 61,670 & 50,000 & 16.0 \\
\hline Oklahoma & 8 & 60,301 & 60,480 & 35,000 & 19.8 \\
\hline Oklahoma State & 8 & 48,833 & 41,208 & 38,000 & 20.3 \\
\hline Pennsylvania & 10 & 60,851 & 58,467 & 42,500 & 15.9 \\
\hline Pennsylvania State & 6 & 63,734 & 61,680 & 42,436 & 16.2 \\
\hline Pittsburgh & 27 & 57,340 & 53,960 & 40,000 & 13.4 \\
\hline Queer`s & 9 & 76,546 & 74,215 & 49,500 & 14.9 \\
\hline Rochester & 24 & 54,807 & 51,777 & 38,168 & 19.1 \\
\hline Saskatchewan & 7 & 64,291 & 61,506 & 49,600 & 7.9 \\
\hline South Carolina & 8 & 48,891 & 45,914 & 35,000 & 12.9 \\
\hline Southern California & 13 & 69,393 & 64,516 & 48,500 & 15.5 \\
\hline Southern Illinois & 6 & 62,521 & 66,592 & 41,000 & 23.5 \\
\hline SUNY Buffalo & 13 & 63,881 & 61,201 & 45,000 & 21.0 \\
\hline SUNY Stony Brook & 18 & 63,156 & 63,412 & 45,000 & 13.4 \\
\hline Temple & 11 & 57,468 & 56,702 & 40,170 & 18.4 \\
\hline Tennessee, Knoxville & 4 & $\neq$ & $\neq$ & 40,000 & 12.0 \\
\hline Tennessee, Memphis & 14 & 53,278 & 50,263 & 45,000 & 17.3 \\
\hline Texas Tech & 21 & 48,703 & 46,271 & 36,000 & 22.7 \\
\hline Toronto & 14 & 96,298 & 106,205 & 51,381 & 19.0 \\
\hline Tulane & 7 & 52,567 & 49,102 & 40,000 & 13.7 \\
\hline Utah & 12 & 62,848 & 59,271 & 35,000 & 19.8 \\
\hline Vanderbilt & 25 & 58,021 & 59,841 & 40,000 & 12.3 \\
\hline Virginia & 15 & 64,952 & 66,000 & 45,000 & 19.4 \\
\hline Washington & 19 & 63,804 & 59,580 & 42,600 & 22.1 \\
\hline Washington U.-St. Louis & 24 & 61,854 & 53,546 & 40,000 & 20.6 \\
\hline Wayne State & 10 & 46,402 & 42,571 & 45,000 & 25.8 \\
\hline Wisconsin & 18 & 61,257 & 56,669 & 40,256 & 12.1 \\
\hline Yale & 21 & 74,991 & 73,264 & 50,500 & 16.0 \\
\hline
\end{tabular}

Directors are included in figures for average years of experience and filled positions, but not in either the average or median salary statistics.

Canadian salaries are expressed in US dollars.

‡ Salary data are not published when fewer than four individuals are involved. 


\section{Table 36: Beginning Professional Salaries in ARL University Medical Libraries RanK Order Table, FY 2008-2009}

\begin{tabular}{|c|c|c|c|c|c|}
\hline Rank & Institution & Salary & Rank & Institution & Salary \\
\hline 1 & Connecticut & $\$ 55,347$ & 36 & Washington & $\$ 42,600$ \\
\hline 2 & British Columbia & 53,021 & 37 & Dartmouth & 42,500 \\
\hline 3 & Columbia & 51,500 & 37 & Pennsylvania & 42,500 \\
\hline 3 & Cornell & 51,500 & 39 & Pennsylvania State & 42,436 \\
\hline 5 & Toronto & 51,381 & 40 & Boston University & 42,000 \\
\hline 6 & Yale & 50,500 & 40 & Florida & 42,000 \\
\hline 7 & Alberta & 50,126 & 40 & Michigan & 42,000 \\
\hline 8 & New York & 50,000 & 43 & Montreal & 41,738 \\
\hline 8 & Ohio State & 50,000 & 44 & Emory & 41,500 \\
\hline 10 & Saskatchewan & 49,600 & 45 & Iowa & 41,000 \\
\hline 11 & McGill & 49,500 & 45 & Kentucky & 41,000 \\
\hline 11 & Queen's & 49,500 & 45 & Northwestern & 41,000 \\
\hline 13 & Harvard & 48,800 & 45 & Southern Illinois & 41,000 \\
\hline 14 & Southern California & 48,500 & 49 & Wisconsin & 40,256 \\
\hline 15 & George Washington & 48,000 & 50 & Temple & 40,170 \\
\hline 16 & Arizona & 47,093 & 51 & Minnesota & 40,000 \\
\hline 17 & Illinois, Chicago & 47,000 & 51 & Missouri & 40,000 \\
\hline 18 & Manitoba & 46,970 & 51 & Pittsburgh & 40,000 \\
\hline 19 & Calif. Davis & 46,164 & 51 & Tennessee, Knoxville & 40,000 \\
\hline 19 & Calif. Irvine & 46,164 & 51 & Tulane & 40,000 \\
\hline 19 & Calif. Los Angeles & 46,164 & 51 & Vanderbilt & 40,000 \\
\hline 19 & Calif. San Diego & 46,164 & 51 & Washington-St. Louis & 40,000 \\
\hline 23 & Howard & 45,000 & 58 & New Mexico & 39,372 \\
\hline 23 & Nebraska & 45,000 & 59 & Cincinnati & 39,000 \\
\hline 23 & North Carolina & 45,000 & 60 & Rochester & 38,168 \\
\hline 23 & SUNY Buffalo & 45,000 & 61 & Florida State & 38,000 \\
\hline 23 & SUNY Stony Brook & 45,000 & 61 & Kansas & 38,000 \\
\hline 23 & Tennessee, Memphis & 45,000 & 61 & Oklahoma State & 38,000 \\
\hline 23 & Virginia & 45,000 & 64 & Louisville & 37,000 \\
\hline 23 & Wayne State & 45,000 & 65 & Texas Tech & 36,000 \\
\hline 31 & Duke & 44,000 & 66 & Alabama & 35,000 \\
\hline 31 & Georgetown & 44,000 & 66 & Case Western Reserve & 35,000 \\
\hline 31 & Johns Hopkins & 44,000 & 66 & Oklahoma & 35,000 \\
\hline 31 & Miami & 44,000 & 66 & South Carolina & 35,000 \\
\hline 35 & Hawaii & 42,649 & 66 & Utah & 35,000 \\
\hline
\end{tabular}

Beginning salary figures represent officially designated base, not necessarily salaries of actual incumbents. Canadian salaries are expressed in US dollars. 


\section{Table 37: Median Professional Salaries in ARL University Medical Libraries \\ RANK ORder TABLE, FY 2008-2009}

\begin{tabular}{|c|c|c|c|c|c|}
\hline Rank & Institution & Salary & Rank & Institution & Salary \\
\hline 1 & Toronto & $\$ 106,205$ & 35 & New York University & $\$ 60,309$ \\
\hline 2 & Alberta & 97,258 & 36 & Vanderbilt & 59,841 \\
\hline 3 & British Columbia & 81,529 & 37 & Washington & 59,580 \\
\hline 4 & Cornell & 78,320 & 38 & Utah & 59,271 \\
\hline 5 & Connecticut & 74,268 & 39 & Georgetown & 59,250 \\
\hline 6 & Queen`s & 74,215 & 40 & Arizona & 58,706 \\
\hline 7 & Harvard & 73,276 & 41 & Pennsylvania & 58,467 \\
\hline 8 & Yale & 73,264 & 42 & Cincinnati & 58,431 \\
\hline 9 & Miami & 72,165 & 43 & Illinois, Chicago & 58,169 \\
\hline 10 & Montreal & 71,761 & 44 & Emory & 57,882 \\
\hline 11 & California, Los Angeles & 68,892 & 45 & Kentucky & 57,641 \\
\hline 12 & Manitoba & 67,995 & 46 & Temple & 56,702 \\
\hline 13 & New Mexico & 67,386 & 47 & Wisconsin & 56,669 \\
\hline 14 & California, San Diego & 66,771 & 48 & Nebraska & 56,644 \\
\hline 15 & McMaster & 66,728 & 49 & Duke & 55,743 \\
\hline 16 & Case Western Reserve & 66,693 & 50 & Louisville & 55,593 \\
\hline 17 & Southern Illinois & 66,592 & 51 & Florida & 55,270 \\
\hline 18 & Virginia & 66,000 & 52 & Michigan & 54,842 \\
\hline 19 & McGill & 65,529 & 53 & Iowa & 54,540 \\
\hline 20 & Southern California & 64,516 & 54 & Pittsburgh & 53,960 \\
\hline 21 & North Carolina & 64,002 & 55 & Washington U.-St. Louis & 53,546 \\
\hline 22 & SUNY Stony Brook & 63,412 & 56 & Howard & 52,009 \\
\hline 23 & Minnesota & 62,692 & 57 & Rochester & 51,777 \\
\hline 24 & Pennsylvania State & 61,680 & 58 & Tennessee, Memphis & 50,263 \\
\hline 25 & Ohio State & 61,670 & 59 & Boston University & 50,000 \\
\hline 26 & Saskatchewan & 61,506 & 60 & Kansas & 49,257 \\
\hline 27 & Northwestern & 61,427 & 61 & Tulane & 49,102 \\
\hline 28 & SUNY Buffalo & 61,201 & 62 & Texas Tech & 46,271 \\
\hline 29 & Columbia & 61,116 & 63 & South Carolina & 45,914 \\
\hline 30 & George Washington & 60,803 & 64 & Hawaii & 45,523 \\
\hline 31 & Johns Hopkins & 60,626 & 65 & Missouri & 43,000 \\
\hline 32 & Oklahoma & 60,480 & 66 & Wayne State & 42,571 \\
\hline 33 & California, Davis & 60,408 & 67 & Oklahoma State & 41,208 \\
\hline 34 & Dartmouth & 60,328 & & & \\
\hline
\end{tabular}

Salaries of directors are not included in the calculation of medians. Alabama, California-Irvine, Florida State, and Tennessee-Knoxville are not ranked because they reported fewer than four individuals.

Canadian salaries are expressed in US dollars. 
Table 38: Average Professional Salaries in ARL University Medical Libraries

Rank Order Table, FY 2008-2009

\begin{tabular}{|c|c|c|c|c|c|}
\hline Rank & Institution & Salary & Rank & Institution & Salary \\
\hline 1 & Alberta & $\$ 96,699$ & 35 & Nebraska & $\$ 62,327$ \\
\hline 2 & Toronto & 96,298 & 36 & Washington U.-St. Louis & 61,854 \\
\hline 3 & California, Davis & 80,311 & 37 & Columbia & 61,564 \\
\hline 4 & Connecticut & 78,122 & 38 & Dartmouth & 61,441 \\
\hline 5 & Cornell & 78,010 & 39 & Wisconsin & 61,257 \\
\hline 6 & British Columbia & 77,805 & 40 & Northwestern & 61,237 \\
\hline 7 & Queen`s & 76,546 & 41 & Pennsylvania & 60,851 \\
\hline 8 & Harvard & 75,515 & 42 & Oklahoma & 60,301 \\
\hline 9 & Yale & 74,991 & 43 & Duke & 60,297 \\
\hline 10 & Manitoba & 72,322 & 44 & George Washington & 59,940 \\
\hline 11 & McGill & 72,309 & 45 & Iowa & 59,648 \\
\hline 12 & Montreal & 72,161 & 46 & Cincinnati & 59,134 \\
\hline 13 & Miami & 71,829 & 47 & Illinois, Chicago & 58,928 \\
\hline 14 & California, Los Angeles & 71,305 & 48 & Florida & 58,902 \\
\hline 15 & New Mexico & 70,363 & 49 & Georgetown & 58,700 \\
\hline 16 & Southern California & 69,393 & 50 & Michigan & 58,250 \\
\hline 17 & Johns Hopkins & 66,744 & 51 & Vanderbilt & 58,021 \\
\hline 18 & California, San Diego & 66,305 & 52 & Kentucky & 57,770 \\
\hline 19 & New York University & 66,047 & 53 & Temple & 57,468 \\
\hline 20 & Ohio State & 66,036 & 54 & Pittsburgh & 57,340 \\
\hline 21 & North Carolina & 65,912 & 55 & Louisville & 57,017 \\
\hline 22 & Virginia & 64,952 & 56 & Boston University & 56,280 \\
\hline 23 & Saskatchewan & 64,291 & 57 & Rochester & 54,807 \\
\hline 24 & Arizona & 64,038 & 58 & Howard & 53,754 \\
\hline 25 & SUNY Buffalo & 63,881 & 59 & Tennessee, Memphis & 53,278 \\
\hline 26 & Washington & 63,804 & 60 & Tulane & 52,567 \\
\hline 27 & Pennsylvania State & 63,734 & 61 & Kansas & 52,333 \\
\hline 28 & Emory & 63,447 & 62 & South Carolina & 48,891 \\
\hline 29 & Minnesota & 63,281 & 63 & Oklahoma State & 48,833 \\
\hline 30 & SUNY Stony Brook & 63,156 & 64 & Texas Tech & 48,703 \\
\hline 31 & Utah & 62,848 & 65 & Hawaii & 46,607 \\
\hline 32 & McMaster & 62,727 & 66 & Wayne State & 46,402 \\
\hline 33 & Case Western Reserve & 62,532 & 67 & Missouri & 45,421 \\
\hline 34 & Southern Illinois & 62,521 & & & \\
\hline
\end{tabular}

Salaries of directors are not included in the calculation of medians. Alabama, California-Irvine, Florida State, and Tennessee-Knoxville are not ranked because they reported fewer than four individuals.

Canadian salaries are expressed in US dollars. 


\section{Table 39: Number and Average Salaries of ARL University Medical Librarians by Position AND SeX, FY 2008-2009}

\begin{tabular}{|c|c|c|c|c|c|c|}
\hline \multirow[b]{2}{*}{ Position } & \multicolumn{2}{|c|}{ WOMEN } & \multicolumn{2}{|c|}{ Men } & \multicolumn{2}{|c|}{ Total } \\
\hline & Salary & No. & Salary & No. & Salary & No. \\
\hline Head, Medical & $\$ 122,689$ & 49 & $\$ 128,373$ & 21 & $\$ 124,394$ & 70 \\
\hline Associate Director & 84,139 & 35 & 92,767 & 10 & 86,056 & 45 \\
\hline Assistant Director & 67,107 & 36 & 74,980 & 7 & 68,389 & 43 \\
\hline Head, Branch & $\neq$ & 28 & $\neq$ & 2 & 70,265 & 30 \\
\hline Functional Specialist & 58,693 & 72 & 60,555 & 79 & 59,667 & 151 \\
\hline Subject Specialist & 61,202 & 68 & 63,723 & 11 & 61,553 & 79 \\
\hline \multicolumn{7}{|l|}{ Dept. Head: } \\
\hline Acquisitions & $\neq$ & 18 & $\neq$ & 3 & 66,689 & 21 \\
\hline Reference & 67,460 & 16 & 74,715 & 10 & 70,250 & 26 \\
\hline Cataloging & $\neq$ & 10 & $\neq$ & 1 & 69,028 & 11 \\
\hline Serials & $\neq$ & 5 & $\neq$ & 1 & 58,052 & 6 \\
\hline Documents/Maps & $\neq$ & 3 & & & $\neq$ & 3 \\
\hline Circulation & 61,651 & 10 & 60,182 & 9 & 60,955 & 19 \\
\hline Rare Books/Manuscripts & $\neq$ & 3 & $\neq$ & 5 & 68,827 & 8 \\
\hline Computer Systems & 72,401 & 9 & 89,998 & 8 & 80,682 & 17 \\
\hline Other & 67,689 & 42 & 74,275 & 13 & 69,246 & 55 \\
\hline \multicolumn{7}{|l|}{ Reference: } \\
\hline Over 14 years experience & 65,798 & 93 & 67,876 & 18 & 66,135 & 111 \\
\hline 10 to 14 years experience & 61,919 & 25 & 62,948 & 12 & 62,253 & 37 \\
\hline 5 to 9 years experience & 57,563 & 49 & 60,340 & 11 & 58,072 & 60 \\
\hline Under 5 years experience & 48,527 & 40 & 45,653 & 9 & 47,999 & 49 \\
\hline \multicolumn{7}{|l|}{ Cataloging: } \\
\hline Over 14 years experience & $\neq$ & 5 & $\neq$ & 2 & 61,626 & 7 \\
\hline 10 to 14 years experience & $\neq$ & 1 & & & $\neq$ & 1 \\
\hline 5 to 9 years experience & $\neq$ & 5 & $\neq$ & 1 & 52,624 & 6 \\
\hline Under 5 years experience & $\neq$ & 3 & . & & 45,734 & 3 \\
\hline \multicolumn{7}{|l|}{ Other: } \\
\hline Over 14 years experience & 63,573 & 26 & 65,211 & 7 & 63,920 & 33 \\
\hline 10 to 14 years experience & 59,142 & 12 & 55,454 & 4 & 58,220 & 16 \\
\hline 5 to 9 years experience & $\neq$ & 12 & $\neq$ & 2 & 55,373 & 14 \\
\hline Under 5 years experience & 46,128 & 13 & 48,697 & 7 & 47,027 & 20 \\
\hline All Positions & $\$ 67,253$ & 688 & $\$ 70,578$ & 253 & $\$ 68,147$ & 941 \\
\hline
\end{tabular}

Canadian salaries are expressed in US dollars.

₹ Salary data are not published when fewer than four individuals are involved in either category. 
Table 40: Number and Average Years of Experience of ARL University Medical Librarians by Position And Sex, FY 2008-2009

\begin{tabular}{|c|c|c|c|c|c|c|}
\hline \multirow[b]{2}{*}{ Position } & \multicolumn{2}{|c|}{ WOMEN } & \multicolumn{2}{|c|}{ Men } & \multicolumn{2}{|c|}{ Total } \\
\hline & Years & No. & Years & No. & Years & No. \\
\hline Head, Medical & 29.1 & 49 & 27.8 & 21 & 28.7 & 70 \\
\hline Associate Director & 23.0 & 35 & 24.6 & 10 & 23.4 & 45 \\
\hline Assistant Director & 18.9 & 36 & 17.7 & 7 & 18.7 & 43 \\
\hline Head, Branch & 20.1 & 28 & 26.5 & 2 & 20.5 & 30 \\
\hline Functional Specialist & 14.1 & 72 & 10.4 & 79 & 12.2 & 151 \\
\hline Subject Specialist & 15.6 & 68 & 12.5 & 11 & 15.2 & 79 \\
\hline \multicolumn{7}{|l|}{ Dept. Head: } \\
\hline Acquisitions & 17.2 & 18 & 21.7 & 3 & 17.8 & 21 \\
\hline Reference & 21.4 & 16 & 23.1 & 10 & 22.0 & 26 \\
\hline Cataloging & 18.6 & 10 & 32.0 & 1 & 19.8 & 11 \\
\hline Serials & 15.2 & 5 & 26.0 & 1 & 17.0 & 6 \\
\hline Documents/Maps & 26.3 & 3 & . & & 26.3 & 3 \\
\hline Circulation & 22.0 & 10 & 8.9 & 9 & 15.8 & 19 \\
\hline Rare Books/Manuscripts & 26.3 & 3 & 26.8 & 5 & 26.6 & 8 \\
\hline Computer Systems & 15.4 & 9 & 22.4 & 8 & 18.7 & 17 \\
\hline Other & 20.0 & 42 & 13.1 & 13 & 18.4 & 55 \\
\hline Public Services & 14.8 & 39 & 9.6 & 14 & 13.4 & 53 \\
\hline Technical Services & 19.2 & 13 & 21.3 & 3 & 19.6 & 16 \\
\hline Administrative Services & 12.6 & 11 & 17.7 & 3 & 13.7 & 14 \\
\hline Reference & 14.4 & 207 & 13.2 & 50 & 14.2 & 257 \\
\hline Cataloger & 11.4 & 14 & 19.7 & 3 & 12.8 & 17 \\
\hline All Positions & 17.3 & 688 & 15.2 & 253 & 16.8 & 941 \\
\hline
\end{tabular}


Table 41: Number and Average Salaries of ARL University Medical Librarians by YeARS OF EXPERIENCE AND SEX, FY 2008-2009

\begin{tabular}{|c|c|c|c|c|c|c|c|}
\hline \multirow[b]{2}{*}{ Experience } & \multicolumn{2}{|c|}{ WOMEN } & \multicolumn{2}{|c|}{ Men } & \multicolumn{2}{|c|}{ TотAL } & \multirow{2}{*}{$\begin{array}{c}\% \text { OF } \\
\text { TotAL }\end{array}$} \\
\hline & Salary & No. & Salary & No. & Salary & No. & \\
\hline $0-3$ years & $\$ 49,923$ & 76 & $\$ 52,525$ & 43 & $\$ 50,863$ & 119 & $13 \%$ \\
\hline $4-7$ years & 56,729 & 92 & 63,830 & 32 & 58,561 & 124 & $13 \%$ \\
\hline $8-11$ years & 60,508 & 93 & 62,967 & 33 & 61,152 & 126 & $13 \%$ \\
\hline 12 - 15 years & 65,709 & 72 & 69,014 & 41 & 66,908 & 113 & $12 \%$ \\
\hline $16-19$ years & 66,442 & 64 & 76,678 & 20 & 68,879 & 84 & $9 \%$ \\
\hline $20-23$ years & 66,023 & 66 & 81,934 & 20 & 69,723 & 86 & $9 \%$ \\
\hline $24-27$ years & 76,480 & 68 & 78,927 & 18 & 76,993 & 86 & $9 \%$ \\
\hline $28-31$ years & 78,503 & 73 & 81,162 & 23 & 79,140 & 96 & $10 \%$ \\
\hline $32-35$ years & 89,832 & 48 & 94,291 & 13 & 90,782 & 61 & $6 \%$ \\
\hline over 35 years & 84,588 & 36 & 96,209 & 10 & 87,114 & 46 & $5 \%$ \\
\hline All Positions & $\$ 67,253$ & 688 & $\$ 70,578$ & 253 & $\$ 68,147$ & 941 & $100 \%$ \\
\hline
\end{tabular}

Canadian salaries are expressed in US dollars. 

ARL UNIVERSITY LAW LIBRARIES

TABLES $42-48$ 


\section{Table 42: Filled Positions; Average, Median, Beginning Professional Salaries; and Average Years of Experience in ARL University LaW Libraries, FY 2008-2009}

\begin{tabular}{|c|c|c|c|c|c|}
\hline Institution & $\begin{array}{c}\text { Filled } \\
\text { Positions }\end{array}$ & $\begin{array}{c}\text { Average } \\
\text { Salary }\end{array}$ & $\begin{array}{c}\text { Median } \\
\text { Salary }\end{array}$ & $\begin{array}{c}\text { Beginning } \\
\text { Salary }\end{array}$ & $\begin{array}{l}\text { Average } \\
\text { Yrs. Exp. }\end{array}$ \\
\hline Alabama & 9 & 58,471 & 57,320 & 52,000 & 10 \\
\hline Alberta & 3 & $\neq$ & $\neq$ & 50,126 & 4 \\
\hline Arizona & 11 & 64,191 & 65,000 & 50,000 & 12 \\
\hline Arizona State & 7 & 67,641 & 73,170 & 43,000 & 8 \\
\hline Boston University & 8 & 71,113 & 63,800 & 55,000 & 9 \\
\hline Boston College & 13 & 75,307 & 80,889 & 42,300 & 14 \\
\hline British Columbia & 2 & $\neq$ & $\neq$ & 53,021 & 3 \\
\hline California, Davis & 6 & 87,438 & 79,974 & 46,164 & 7 \\
\hline California, Los Angeles & 14 & 78,124 & 82,524 & 46,164 & 15 \\
\hline Case Western Reserve & 10 & 64,326 & 65,931 & 35,000 & 11 \\
\hline Cincinnati & 5 & 60,748 & 52,020 & 42,298 & 6 \\
\hline Colorado & 6 & 70,199 & 58,602 & 45,000 & 7 \\
\hline Columbia & 15 & 76,365 & 73,672 & 51,500 & 16 \\
\hline Connecticut & 9 & 70,729 & 71,752 & 45,000 & 10 \\
\hline Cornell & 7 & 76,857 & 71,400 & 58,000 & 8 \\
\hline Duke & 10 & 74,075 & 68,575 & 55,000 & 11 \\
\hline Emory & 9 & 61,465 & 58,984 & 41,500 & 10 \\
\hline Florida & 9 & 59,992 & 54,968 & 50,000 & 10 \\
\hline Florida State & 9 & 53,794 & 51,500 & 52,000 & 10 \\
\hline George Washington & 19 & 83,872 & 78,246 & 59,000 & 20 \\
\hline Georgetown & 24 & 74,448 & 70,000 & 46,500 & 25 \\
\hline Georgia & 7 & 55,235 & 52,844 & 40,000 & 8 \\
\hline Harvard & 42 & 78,934 & 73,409 & 48,800 & 43 \\
\hline Hawaii & 3 & $\neq$ & $\neq$ & 50,000 & 4 \\
\hline Houston & 10 & 57,406 & 52,790 & 50,000 & 11 \\
\hline Howard & 6 & 49,623 & 50,688 & 45,000 & 7 \\
\hline Illinois, Urbana & 9 & 62,773 & 59,129 & 53,500 & 10 \\
\hline Indiana & 9 & 69,325 & 64,923 & 40,400 & 10 \\
\hline Iowa & 16 & 72,925 & 69,625 & 50,000 & 17 \\
\hline Kansas & 7 & 49,278 & 44,555 & 35,000 & 8 \\
\hline Kentucky & 6 & 51,515 & 46,338 & 41,000 & 7 \\
\hline Louisiana State & 10 & 60,863 & 55,846 & 42,000 & 11 \\
\hline Louisville & 5 & 56,866 & 53,853 & 37,000 & 6 \\
\hline McGill & 3 & $\neq$ & $\neq$ & 49,500 & 4 \\
\hline Manitoba & 2 & $\neq$ & $\neq$ & 46,970 & 3 \\
\hline Miami & 11 & 58,981 & 58,213 & 44,000 & 12 \\
\hline Michigan & 11 & 76,256 & 70,553 & 49,000 & 12 \\
\hline Minnesota & 13 & 75,486 & 68,200 & 44,000 & 14 \\
\hline Missouri & 7 & 54,645 & 57,327 & N/A & 8 \\
\hline
\end{tabular}




\section{Table 42: Filled Positions; Average, Median, Beginning Professional Salaries; and Average Years of Experience \\ in ARL University LaW LibraRies, FY 2008-2009}

\begin{tabular}{|c|c|c|c|c|c|}
\hline Institution & $\begin{array}{c}\text { Filled } \\
\text { Positions }\end{array}$ & $\begin{array}{c}\text { Average } \\
\text { Salary }\end{array}$ & $\begin{array}{c}\text { Median } \\
\text { Salary }\end{array}$ & $\begin{array}{c}\text { Beginning } \\
\text { Salary }\end{array}$ & $\begin{array}{l}\text { Average } \\
\text { Yrs. Exp. }\end{array}$ \\
\hline Montreal & 4 & 65,499 & 60,171 & 51,277 & 5 \\
\hline Nebraska & 4 & 62,615 & 62,417 & 43,000 & 5 \\
\hline New Mexico & 6 & 64,180 & 56,927 & 49,000 & 7 \\
\hline New York University & 17 & 82,580 & 80,845 & 60,000 & 18 \\
\hline North Carolina & 11 & 74,947 & 68,000 & 45,000 & 12 \\
\hline Northwestern & 10 & 62,529 & 58,845 & 44,000 & 11 \\
\hline Notre Dame & 12 & 69,321 & 64,300 & 40,000 & 13 \\
\hline Ohio State & 6 & 64,224 & 60,197 & 42,000 & 7 \\
\hline Oklahoma & 6 & 55,874 & 49,897 & 42,000 & 7 \\
\hline Oregon & 5 & 60,799 & 58,995 & 42,000 & 6 \\
\hline Pennsylvania & 13 & 70,077 & 63,978 & 42,500 & 14 \\
\hline Pennsylvania State & 9 & 75,059 & 69,360 & 42,436 & 10 \\
\hline Queen`s & 2 & $\neq$ & $\neq$ & 49,500 & 3 \\
\hline Rutgers, Camden & 7 & 77,533 & 85,700 & 52,000 & 8 \\
\hline Rutgers, Newark & 9 & 72,346 & 64,000 & 52,000 & 10 \\
\hline Saskatchewan & 2 & 94,209 & 94,209 & 49,600 & 3 \\
\hline South Carolina & 9 & 67,341 & 68,289 & 50,000 & 10 \\
\hline Southern Illinois & 3 & $\neq$ & $\neq$ & 50,000 & 4 \\
\hline SUNY Buffalo & 10 & 69,492 & 74,223 & 45,000 & 11 \\
\hline Syracuse & 9 & 58,398 & 55,146 & 46,000 & 10 \\
\hline Temple & 8 & 63,612 & 54,635 & 41,616 & 9 \\
\hline Tennessee & 8 & 67,824 & 57,719 & 50,000 & 9 \\
\hline Texas & 12 & 60,540 & 55,857 & 38,000 & 13 \\
\hline Texas Tech & 6 & 65,190 & 63,747 & 46,500 & 7 \\
\hline Toronto & 3 & $\neq$ & $\neq$ & 51,381 & 4 \\
\hline Tulane & 6 & 57,587 & 56,970 & 40,000 & 7 \\
\hline Utah & 7 & 56,136 & 52,530 & 45,000 & 8 \\
\hline Vanderbilt & 5 & 66,594 & 60,355 & 40,000 & 6 \\
\hline Virginia & 12 & 64,842 & 61,350 & 62,500 & 13 \\
\hline Washington & 14 & 69,248 & 67,879 & 57,000 & 15 \\
\hline Washington U.-St. Louis & 7.0 & $115,897.0$ & $71,667.0$ & 53,500 & 8 \\
\hline Wayne State & 8 & 45,730 & 44,242 & 45,000 & 9 \\
\hline Western Ontario & 3 & $\neq$ & $\neq$ & 46,906 & 4 \\
\hline Wisconsin & 11 & 62,919 & 58,954 & 40,256 & 12 \\
\hline Yale & 18 & 80,486 & 75,612 & 50,500 & 19 \\
\hline York & 4 & 81,778 & 83,597 & 48,510 & 5 \\
\hline
\end{tabular}

Directors are included in figures for average years of experience and filled positions, but not in either the average or median salary statistics.

Canadian salaries are expressed in US dollars.

‡ Salary data are not published when fewer than four individuals are involved. 


\section{Table 43: Beginning Professional Salaries in ARL University Law Libraries RANK ORder Table, FY 2008-2009}

\begin{tabular}{|c|c|c|c|c|c|}
\hline Rank & Institution & Salary & Rank & Institution & Salary \\
\hline 1 & Virginia & 62,500 & 39 & Calif. Davis & 46,164 \\
\hline 2 & New York & 60,000 & 39 & Calif. Los Angeles & 46,164 \\
\hline 3 & George Washington & 59,000 & 41 & Syracuse & 46,000 \\
\hline 4 & Cornell & 58,000 & 42 & Colorado & 45,000 \\
\hline 5 & Washington & 57,000 & 42 & Connecticut & 45,000 \\
\hline 6 & Boston University & 55,000 & 42 & Howard & 45,000 \\
\hline 6 & Duke & 55,000 & 42 & North Carolina & 45,000 \\
\hline 8 & Illinois, Urbana & 53,500 & 42 & SUNY Buffalo & 45,000 \\
\hline 8 & Washington-St. Louis & 53,500 & 42 & Utah & 45,000 \\
\hline 10 & British Columbia & 53,021 & 42 & Wayne State & 45,000 \\
\hline 11 & Alabama & 52,000 & 49 & Miami & 44,000 \\
\hline 11 & Florida State & 52,000 & 49 & Minnesota & 44,000 \\
\hline 11 & Rutgers, Camden Law & 52,000 & 49 & Northwestern & 44,000 \\
\hline 11 & Rutgers, Newark Law & 52,000 & 52 & Arizona State & 43,000 \\
\hline 15 & Columbia & 51,500 & 52 & Nebraska & 43,000 \\
\hline 16 & Toronto & 51,381 & 54 & Pennsylvania & 42,500 \\
\hline 17 & Montreal & 51,277 & 55 & Pennsylvania State & 42,436 \\
\hline 18 & Yale & 50,500 & 56 & Boston College & 42,300 \\
\hline 19 & Alberta & 50,126 & 57 & Cincinnati & 42,298 \\
\hline 20 & Arizona & 50,000 & 58 & Louisiana State & 42,000 \\
\hline 20 & Florida & 50,000 & 58 & Ohio State & 42,000 \\
\hline 20 & Hawaii & 50,000 & 58 & Oklahoma & 42,000 \\
\hline 20 & Houston & 50,000 & 58 & Oregon & 42,000 \\
\hline 20 & Iowa & 50,000 & 62 & Temple & 41,616 \\
\hline 20 & South Carolina & 50,000 & 63 & Emory & 41,500 \\
\hline 20 & Southern Illinois & 50,000 & 64 & Kentucky & 41,000 \\
\hline 20 & Tennessee & 50,000 & 65 & Indiana & 40,400 \\
\hline 28 & Saskatchewan & 49,600 & 66 & Wisconsin & 40,256 \\
\hline 29 & McGill & 49,500 & 67 & Georgia & 40,000 \\
\hline 29 & Queen's & 49,500 & 67 & Notre Dame & 40,000 \\
\hline 31 & Michigan & 49,000 & 67 & Tulane & 40,000 \\
\hline 31 & New Mexico & 49,000 & 67 & Vanderbilt & 40,000 \\
\hline 33 & Harvard & 48,800 & 71 & Texas & 38,000 \\
\hline 34 & York & 48,510 & 72 & Louisville & 37,000 \\
\hline 35 & Manitoba & 46,970 & 73 & Case Western Reserve & 35,000 \\
\hline 36 & Western Ontario & 46,906 & 73 & Kansas & 35,000 \\
\hline 37 & Georgetown & 46,500 & & Missouri & $\mathrm{N} / \mathrm{A}$ \\
\hline 37 & Texas Tech & 46,500 & & & \\
\hline
\end{tabular}

Beginning salary figures represent officially designated base, not necessarily salaries of actual incumbents. Canadian salaries are expressed in US dollars. 


\section{Table 44: Median Professional Salaries in ARL University Law Libraries \\ Rank Order Table, FY 2008-2009}

\begin{tabular}{|c|c|c|c|c|c|}
\hline Rank & Institution & Salary & Rank & Institution & Salary \\
\hline 1 & Saskatchewan & 94,209 & 34 & Nebraska & 62,417 \\
\hline 2 & Rutgers, Camden & 85,700 & 35 & Virginia & 61,350 \\
\hline 3 & York & 83,597 & 36 & Vanderbilt & 60,355 \\
\hline 4 & California, Los Angeles & 82,524 & 37 & Ohio State & 60,197 \\
\hline 5 & Boston College & 80,889 & 38 & Montreal & 60,171 \\
\hline 6 & New York University & 80,845 & 39 & Illinois, Urbana & 59,129 \\
\hline 7 & California, Davis & 79,974 & 40 & Oregon & 58,995 \\
\hline 8 & George Washington & 78,246 & 41 & Emory & 58,984 \\
\hline 9 & Yale & 75,612 & 42 & Wisconsin & 58,954 \\
\hline 10 & SUNY Buffalo & 74,223 & 43 & Northwestern & 58,845 \\
\hline 11 & Columbia & 73,672 & 44 & Colorado & 58,602 \\
\hline 12 & Harvard & 73,409 & 45 & Miami & 58,213 \\
\hline 13 & Arizona State & 73,170 & 46 & Tennessee & 57,719 \\
\hline 14 & Connecticut & 71,752 & 47 & Missouri & 57,327 \\
\hline 15 & Washington U.-St. Louis & 71,667 & 48 & Alabama & 57,320 \\
\hline 16 & Cornell & 71,400 & 49 & Tulane & 56,970 \\
\hline 17 & Michigan & 70,553 & 50 & New Mexico & 56,927 \\
\hline 18 & Georgetown & 70,000 & 51 & Texas & 55,857 \\
\hline 19 & Iowa & 69,625 & 52 & Louisiana State & 55,846 \\
\hline 20 & Pennsylvania State & 69,360 & 53 & Syracuse & 55,146 \\
\hline 21 & Duke & 68,575 & 54 & Florida & 54,968 \\
\hline 22 & South Carolina & 68,289 & 55 & Temple & 54,635 \\
\hline 23 & Minnesota & 68,200 & 56 & Louisville & 53,853 \\
\hline 24 & North Carolina & 68,000 & 57 & Georgia & 52,844 \\
\hline 25 & Washington & 67,879 & 58 & Houston & 52,790 \\
\hline 26 & Case Western Reserve & 65,931 & 59 & Utah & 52,530 \\
\hline 27 & Arizona & 65,000 & 60 & Cincinnati & 52,020 \\
\hline 28 & Indiana & 64,923 & 61 & Florida State & 51,500 \\
\hline 29 & Notre Dame & 64,300 & 62 & Howard & 50,688 \\
\hline 30 & Rutgers, Newark & 64,000 & 63 & Oklahoma & 49,897 \\
\hline 31 & Pennsylvania & 63,978 & 64 & Kentucky & 46,338 \\
\hline 32 & Boston University & 63,800 & 65 & Kansas & 44,555 \\
\hline 33 & Texas Tech & 63,747 & 66 & Wayne State & 44,242 \\
\hline
\end{tabular}

Salaries of directors are not included in the calculation of medians. Alberta, British Columbia, Hawaii, McGill, Manitoba, Queen's, Saskatchewan, Southern Illinois, Toronto, and Western Ontario are not ranked because they reported fewer than four individuals. Canadian salaries are expressed in US dollars. 


\section{Table 45: Average Professional Salaries in ARL University Law Libraries \\ Rank Order Table, FY 2008-2009}

\begin{tabular}{|c|c|c|c|c|c|}
\hline Rank & Institution & Salary & Rank & Institution & Salary \\
\hline 1 & Washington U.-St. Louis & 115,897 & 34 & Montreal & 65,499 \\
\hline 2 & Saskatchewan & 94,209 & 35 & Texas Tech & 65,190 \\
\hline 3 & California, Davis & 87,438 & 36 & Virginia & 64,842 \\
\hline 4 & George Washington & 83,872 & 37 & Case Western Reserve & 64,326 \\
\hline 5 & New York University & 82,580 & 38 & Ohio State & 64,224 \\
\hline 6 & York & 81,778 & 39 & Arizona & 64,191 \\
\hline 7 & Yale & 80,486 & 40 & New Mexico & 64,180 \\
\hline 8 & Harvard & 78,934 & 41 & Temple & 63,612 \\
\hline 9 & California, Los Angeles & 78,124 & 42 & Wisconsin & 62,919 \\
\hline 10 & Rutgers, Camden & 77,533 & 43 & Illinois, Urbana & 62,773 \\
\hline 11 & Cornell & 76,857 & 44 & Nebraska & 62,615 \\
\hline 12 & Columbia & 76,365 & 45 & Northwestern & 62,529 \\
\hline 13 & Michigan & 76,256 & 46 & Emory & 61,465 \\
\hline 14 & Minnesota & 75,486 & 47 & Louisiana State & 60,863 \\
\hline 15 & Boston College & 75,307 & 48 & Oregon & 60,799 \\
\hline 16 & Pennsylvania State & 75,059 & 49 & Cincinnati & 60,748 \\
\hline 17 & North Carolina & 74,947 & 50 & Texas & 60,540 \\
\hline 18 & Georgetown & 74,448 & 51 & Florida & 59,992 \\
\hline 19 & Duke & 74,075 & 52 & Miami & 58,981 \\
\hline 20 & Iowa & 72,925 & 53 & Alabama & 58,471 \\
\hline 21 & Rutgers, Newark & 72,346 & 54 & Syracuse & 58,398 \\
\hline 22 & Boston University & 71,113 & 55 & Tulane & 57,587 \\
\hline 23 & Connecticut & 70,729 & 56 & Houston & 57,406 \\
\hline 24 & Colorado & 70,199 & 57 & Louisville & 56,866 \\
\hline 25 & Pennsylvania & 70,077 & 58 & Utah & 56,136 \\
\hline 26 & SUNY Buffalo & 69,492 & 59 & Oklahoma & 55,874 \\
\hline 27 & Indiana & 69,325 & 60 & Georgia & 55,235 \\
\hline 28 & Notre Dame & 69,321 & 61 & Missouri & 54,645 \\
\hline 29 & Washington & 69,248 & 62 & Florida State & 53,794 \\
\hline 30 & Tennessee & 67,824 & 63 & Kentucky & 51,515 \\
\hline 31 & Arizona State & 67,641 & 64 & Howard & 49,623 \\
\hline 32 & South Carolina & 67,341 & 65 & Kansas & 49,278 \\
\hline 33 & Vanderbilt & 66,594 & 66 & Wayne State & 45,730 \\
\hline
\end{tabular}

Salaries of directors are not included in the calculation of medians. Alberta, British Columbia, Hawaii, McGill, Manitoba, Queen's, Saskatchewan, Southern Illinois, Toronto, and Western Ontario are not ranked because they reported fewer than four individuals. Canadian salaries are expressed in US dollars. 


\section{Table 46: Number and Average Salaries of ARL University Law Librarians by Position AND SeX, FY 2008-2009}

\begin{tabular}{|c|c|c|c|c|c|c|}
\hline \multirow[b]{2}{*}{ Position } & \multicolumn{2}{|c|}{ WOMEN } & \multicolumn{2}{|c|}{ MeN } & \multicolumn{2}{|c|}{ Total } \\
\hline & Salary & No. & Salary & No. & Salary & No. \\
\hline Head, Law & $\$ 149,978$ & 36 & $\$ 154,892$ & 39 & $\$ 152,533$ & 75 \\
\hline Associate Director & 99,467 & 30 & 96,404 & 20 & 98,242 & 50 \\
\hline Assistant Director & 84,936 & 32 & 83,761 & 9 & 84,678 & 41 \\
\hline Functional Specialist & 58,079 & 32 & 62,141 & 25 & 59,861 & 57 \\
\hline Subject Specialist & 72,539 & 29 & 71,527 & 15 & 72,194 & 44 \\
\hline \multicolumn{7}{|l|}{ Dept. Head: } \\
\hline Acquisitions & 62,897 & 25 & 61,692 & 9 & 62,578 & 34 \\
\hline Reference & 76,890 & 19 & 75,994 & 10 & 76,581 & 29 \\
\hline Cataloging & $\neq$ & 24 & ‡ & 3 & 73,177 & 27 \\
\hline Serials & $\neq$ & 8 & $\neq$ & 2 & 69,322 & 10 \\
\hline Documents/Maps & $\neq$ & 9 & $\neq$ & 1 & 65,235 & 10 \\
\hline Circulation & 60,794 & 23 & 60,383 & 7 & 60,698 & 30 \\
\hline Rare Books/Manuscripts & $\neq$ & 3 & $\neq$ & 2 & 75,572 & 5 \\
\hline Computer Systems & 69,891 & 4 & 77,110 & 5 & 73,901 & 9 \\
\hline Other & 71,998 & 23 & 73,150 & 10 & 72,347 & 33 \\
\hline \multicolumn{7}{|l|}{ Reference: } \\
\hline Over 14 years experience & 85,220 & 40 & 72,586 & 22 & 80,737 & 62 \\
\hline 10 to 14 years experience & 61,245 & 19 & 61,141 & 9 & 61,211 & 28 \\
\hline 5 to 9 years experience & 58,863 & 19 & 61,282 & 14 & 59,889 & 33 \\
\hline Under 5 years experience & 59,217 & 52 & 57,025 & 28 & 58,450 & 80 \\
\hline \multicolumn{7}{|l|}{ Cataloging: } \\
\hline Over 14 years experience & 63,539 & 20 & 70,213 & 7 & 65,269 & 27 \\
\hline 10 to 14 years experience & $\neq$ & 9 & $\neq$ & 1 & 58,809 & 10 \\
\hline 5 to 9 years experience & 52,391 & 7 & . & & 52,391 & 7 \\
\hline Under 5 years experience & 54,834 & 7 & . & & 54,834 & 7 \\
\hline \multicolumn{7}{|l|}{ Other: } \\
\hline Over 14 years experience & 63,296 & 9 & 64,900 & 5 & 63,869 & 14 \\
\hline 10 to 14 years experience & $\neq$ & 6 & $\neq$ & 1 & 68,322 & 7 \\
\hline 5 to 9 years experience & $\neq$ & 3 & $\neq$ & 4 & 54,694 & 7 \\
\hline Under 5 years experience & $\neq$ & 6 & $\neq$ & 1 & 52,051 & 7 \\
\hline All Positions & $\$ 75,619$ & 494 & $\$ 82,613$ & 249 & $\$ 77,963$ & 743 \\
\hline
\end{tabular}

Canadian salaries are expressed in US dollars.

₹ Salary data are not published when fewer than four individuals are involved in either category. 

Table 47: Number and Average Years of Experience OF ARL University LaW Librarians
By Position And Sex, FY 2008-2009

\begin{tabular}{|c|c|c|c|c|c|c|}
\hline \multirow[b]{2}{*}{ Position } & \multicolumn{2}{|c|}{ Women } & \multicolumn{2}{|c|}{ Men } & \multicolumn{2}{|c|}{ TOTAL } \\
\hline & Years & No. & Years & No. & Years & No. \\
\hline Head, Law & 29.1 & 36 & 23.1 & 39 & 26.0 & 75 \\
\hline Associate Director & 24.8 & 30 & 19.8 & 20 & 22.8 & 50 \\
\hline Assistant Director & 21.7 & 32 & 20.8 & 9 & 21.5 & 41 \\
\hline Functional Specialist & 12.9 & 32 & 10.0 & 25 & 11.6 & 57 \\
\hline Subject Specialist & 20.3 & 29 & 19.1 & 15 & 19.9 & 44 \\
\hline \multicolumn{7}{|l|}{ Dept. Head: } \\
\hline Acquisitions & 19.6 & 25 & 17.2 & 9 & 19.0 & 34 \\
\hline Reference & 14.9 & 19 & 18.6 & 10 & 16.2 & 29 \\
\hline Cataloging & 26.5 & 24 & 18.0 & 3 & 25.5 & 27 \\
\hline Serials & 17.8 & 8 & 11.0 & 2 & 16.4 & 10 \\
\hline Documents/Maps & 28.2 & 9 & 11.0 & 1 & 26.5 & 10 \\
\hline Circulation & 14.9 & 23 & 10.7 & 7 & 13.9 & 30 \\
\hline Rare Books/Manuscripts & 15.0 & 3 & 13.5 & 2 & 14.4 & 5 \\
\hline Computer Systems & 22.0 & 4 & 17.4 & 5 & 19.4 & 9 \\
\hline Other & 17.7 & 23 & 16.8 & 10 & 17.4 & 33 \\
\hline Public Services & 13.7 & 6 & 13.2 & 6 & 13.4 & 12 \\
\hline Technical Services & 12.8 & 12 & 12.0 & 3 & 12.6 & 15 \\
\hline Administrative Services & 18.5 & 6 & 20.0 & 2 & 18.9 & 8 \\
\hline Reference & 11.6 & 130 & 10.9 & 73 & 11.4 & 203 \\
\hline Cataloger & 17.5 & 43 & 24.9 & 8 & 18.7 & 51 \\
\hline All Positions & 17.8 & 494 & 15.9 & 249 & 17.1 & 743 \\
\hline
\end{tabular}


Table 48: Number and Average Salaries of ARL University Law Librarians by YeARS OF EXPERIENCE AND SEX, FY 2008-2009

\begin{tabular}{lccccccc}
\hline & \multicolumn{2}{c}{ WoMEN } & \multicolumn{2}{c}{ MeN } & \multicolumn{2}{c}{ Total } & \% OF \\
Experience & Salary & No. & Salary & No. & Salary & No. & TotaL \\
\hline $0-3$ years & $\$ 56,389$ & 68 & $\$ 54,301$ & 31 & $\$ 55,735$ & 99 & $13 \%$ \\
$4-7$ years & 59,363 & 67 & 63,628 & 34 & 60,799 & 101 & $14 \%$ \\
$8-11$ years & 68,693 & 38 & 67,505 & 34 & 68,132 & 72 & $10 \%$ \\
$12-15$ years & 68,524 & 65 & 89,699 & 29 & 75,057 & 94 & $13 \%$ \\
$16-19$ years & 76,601 & 41 & 84,232 & 23 & 79,343 & 64 & $9 \%$ \\
$20-23$ years & 95,389 & 36 & 85,983 & 36 & 90,686 & 72 & $10 \%$ \\
$24-27$ years & 79,165 & 43 & 110,736 & 16 & 87,726 & 59 & $8 \%$ \\
$28-31$ years & 85,651 & 54 & 105,363 & 25 & 91,889 & 79 & $11 \%$ \\
$32-35$ years & 98,760 & 54 & 104,743 & 17 & 100,192 & 71 & $10 \%$ \\
over 35 years & 90,804 & 28 & 152,071 & 4 & 98,462 & 32 & $4 \%$ \\
\hline All Positions & $\$ 75,619$ & 494 & $\$ 82,613$ & $\mathbf{2 4 9}$ & $\$ 77,963$ & 743 & $\mathbf{1 0 0 \%}$ \\
\hline
\end{tabular}

Canadian salaries are expressed in US dollars. 

UNIVERSITY LiBRARY QUESTIONNAIRE AND INSTRUCTIONS 


\section{ARL Annual Salary Survey 2008-2009 \\ University Library Questionnaire \\ General And Data InPUt (Excel) Instructions}

\section{http://www.arl.org/stats/annualsurveys/salary/}

\section{General Overview}

- Use the newly available Web form for your data submission:

- University Libraries: http://www.formspring.com/forms/?366101-c6BynijupA

- Nonuniversity Libraries: http://www.formspring.com/forms/?366133-c6BynijupA Fill in Part I on the Web and upload your file for Part II through the same interface.

NOTE: You must complete the entire submission in a single session. The Web interface does NOT allow you to return and edit your information once it is submitted.

- This survey is concerned with professional positions only. Since the criteria for determining professional status vary among libraries, there is no attempt to define the term "professional." Each library should report the salaries of those staff members it considers professionals, irrespective of faculty status or membership in a collective bargaining unit, including, when appropriate, staff who are not librarians in the strict sense of the term, such as computer experts, systems analysts, budget officers, etc.

- Report individual salaries for the Main, Law, and Medical library on the separate template using Microsoft Excel (see http://www.arl.org/stats/annualsurveys/salary/salform08.shtml). A generic template is available. Add your institution's ARL Library Institution Code [LibID]. (See http://www.arl.org/stats/ annualsurveys/surveycoord/instno_inam.shtml if you do not know your code.)

- Use "Percent" to determine if an employee works full-time or part-time. All full-time employees have Percent $=1.00$, i.e., they work $100 \%$ of a full-time schedule. If Percent is less than 1.00 , then the employee works that fraction of a full-time schedule. For example, a $65 \%$ time appointment would be entered as 0.65 . Calculate the percent appointment by dividing the amount of time an employee works by the amount considered to be the norm for full-time employment at your institution. For example, if a full-time appointment at your institution is 12 months at 40 hours per week:

- A 9-month part-time appointment has Percent $=9 / 12$, or 0.75 .

- An appointment at 30 hours per week has Percent $=30 / 40$, also 0.75 .

○ An appointment at 30 hours and 9 months has Percent $=0.75 \times 0.75=0.56$.

Enter Percent with two decimal points.

- Report salaries for both full-time and part-time professional positions. Salaries for part-time positions should NOT be converted to their full-time equivalents. Report the actual part-time salary paid and indicate the percent appointment for that employee in the appropriate column.

- Include salaries for all professional positions, regardless of whether the salaries come from regular library budget funds or from special funds such as research grants. Please include all professionals involved in the provision of library services, including contract-supported positions.

- The salary figures should be straight gross salary figures. Do not include fringe benefits. 
- Provide explanatory footnotes to the reported figures, when necessary, at the end of Part I. Footnotes will be included in the published survey, where appropriate.

- After all data have been entered, make a backup copy of the complete file for your institution's master file. Your backup should include individual names/ID numbers. NOTE: The data submitted to ARL should NOT include individual names/ID numbers, so ARL will NOT be able to supply a copy of your institution's complete file next year.

- Please return the questionnaire the ARL Statistics and Measurement Program by September 30, 2008. Be sure to keep a complete copy of your return, including the electronic version of the data for your files.

\section{INSTRUCTIONS}

\section{Part I: Summary Data}

1. Part I of this survey deals with general information for the current fiscal year, 2008-2009.

2. Include the Beginning Professional Salary for Law and Medical libraries if included in the survey.

3. The Beginning Professional Salary is the salary that would be paid to a newly hired professional without experience, not necessarily the lowest professional salary paid. In reporting the beginning salary, please use a figure that is actually used or likely to be used for entry-level librarians hired by your library, even if it is your practice rarely to hire entry-level professionals without experience.

4. Please report the 2008-2009 Beginning Professional Salary to the best of your knowledge as it exists on July 1 , 2008. Do not delay returning your survey with the expectation that more information will be available later.

5. The 2008-2009 Average and Median Salary figures will be calculated by ARL from the individual data supplied.

6. Be sure to fill in the name of the reporting library and the name of the person who prepares the report.

\section{Part II: Individual Data}

1. Part II of this survey requests information on salary, sex, minority status, rank, and years of experience for all filled positions for fiscal year 2008-2009. The survey requests information for individuals; aggregate data for each institution will be generated by computer. Vacant positions should be excluded from your report.

2. Data for the Main, Law, and Medical libraries should be reported on separate Excel files.

3. Obtain the Excel file. These instructions assume that you have Microsoft Excel available for use. If not, or if you have trouble opening the files in Excel, please call the ARL Statistics and Measurement Program at (202) 296-2296 or email stats@arl.org. 
4. The template Excel file is available at: http://www.arl.org/stats/annualsurveys/salary/salform08.shtml. This is a generic, blank file that can hold data for Main, Law, or Medical libraries. The file's name is "sal08xxxx. xls"; open the file and save it to your own computer by choosing "Save As" under the File menu. When saving the file, please change the "xxxx" in its name to your institution's name, e.g., "sal08ALABAMA.xls."

The file contains columns labeled as follows:

Required: Name/ID\# LibID Page Line Salary Job Sex OEOcat Yrsexp Rank Percent

Optional: Hisp NatAm Asian Black HawPI White

In the LibID column, enter your ARL Library Institution Code. (See http://www.arl.org/stats/ annualsurveys/surveycoord/instno_inam.shtml if you do not know your code.) If you leave this column blank we will fill it in for you when we receive the data.

Columns labeled "Page," "Line," and "Percent" are already filled for you. The numbers in the "Page" and "Line" columns will be used to identify these positions in case of data errors; do not change them. Ten "pages" of 25 lines each have been provided; if this is not sufficient to list all positions at your institution, copy and paste lines $1-25$ of the last page as needed.

\section{Entering Data for Part II: Individual Data}

1. The "Name/ID\#" column is for your internal use, to enter and verify information for staff members by name. ARL does not require that you submit the information in this field to ARL. Please delete this column before sending the file to ARL. Upon receiving this file, ARL will delete any data in this column if you have not deleted them already.

2. The "LibID" will hold your institution's ARL number, for identification purposes. If you do not know your ARL number, you can find it on the Web under ARL Library Institution Codes. If you leave this column blank, it will be filled in by ARL staff.

3. "Salary" should be entered as it existed on July 1, 2008. Please do not hold up the reporting process for later salary adjustments. Include all filled positions and exclude all vacant positions. Report the actual salary paid. Do not adjust part-time salaries to their full-time equivalents; ARL will do this during the data analysis and verification stage. Do not include fringe benefits.

4. Each position can have only one "Job" code, to be taken from the following list:

DIRLIB Director of Libraries (includes Dean of Libraries and equivalent titles)

ASCDIR Associate Director

ASTDIR Assistant Director

HDMED Head, Medical Library (Human Medicine only)

HDLAW Head, Law Library

HDBR Head, Other Branch Library (including Veterinary Medicine)

FSPEC Functional Specialist

ARCH Archivists/Curators

BUSI Budget/Fiscal/Business Manager/Facilities

HUMRES Human Resources/Training/Staff Development

ITS Information Technology Systems 


\begin{tabular}{|c|c|}
\hline ITW & Information Technology Web Development \\
\hline ITP & Information Technology Programming/Application Development \\
\hline \multicolumn{2}{|r|}{ MEDIA Media/Multimedia Specialists (including graphics) } \\
\hline PRES & Preservation/Conservation \\
\hline SSPEC & Subject Specialist \\
\hline HDACQ & Head, Acquisitions Department \\
\hline HDCAT & Head, Catalog Department/Unit \\
\hline HDCIRC & Head, Circulation \\
\hline HDCOMP & Head, Library and Computer Systems \\
\hline HDDOC & Head, Documents Department \\
\hline HDMAP & Head, Map Room/Department \\
\hline HDRBM & Head, Rare Book/Manuscripts Department \\
\hline HDREF & Head, Reference Department \\
\hline HDSER & Head, Serials Department \\
\hline HDOTH & Head, Other Department/Service/Agency \\
\hline CAT & Catalogers, both general and specialized \\
\hline REF & Reference librarians, both general and specialized \\
\hline PUBS & Public Services, non-supervisory, except reference librarians \\
\hline TECH & Technical Services, non-supervisory, except catalogers \\
\hline ADMIN & Administrative and other units, non-supervisory position \\
\hline
\end{tabular}

The position categories used in this survey are intended to correspond roughly with the activities carried on in libraries, not with any particular pattern of staff organization or nomenclature. Please use these categories in the manner you feel best applies to your library. If any individual has responsibilities described by more than one of the above categories, choose the category that is most typical of his/her general duties.

Associate or Assistant Director, and Head, Other Branch. Use these codes for all persons at these levels regardless of the area of specialty. If an assistant or associate director is also head of a department, choose the category that most reflects the general duties of the person currently in the position.

Specialists. These are of two kinds: Subject Specialists primarily build collections, but may also offer specialized reference and bibliographic services; Functional Specialists are media specialists or experts in management fields such as personnel, fiscal matters, systems, preservation, etc. Specialists may not be, strictly speaking, professional librarians (i.e., have an MLS). The "specialist" category would generally not be used for someone with significant supervisory responsibilities, who should instead be listed as a department head or assistant director (see also note under Assistant Department Head, below).

Functional Specialist sub-codes. Starting with the 2004-05 Salary Survey, the ARL Statistics and Measurement Committee adopted a proposal from the ACRL Personnel Administrators and Staff Development Officers Discussion Group to break down the Functional Specialist category. For each position which would have been labeled FSPEC prior to 2004-05, instead please use one of the eight sub-codes (ARCH, BUSI, HUMRES, ITS, ITW, ITP, MEDIA, PRES) to describe that position. If you cannot determine which sub-code to use, please use the FSPEC code.

Department Heads. Department Heads not specifically included in the above list should be included under the category "Head, Other Department/Service/Agency." Head, Catalog Department should be used either for the 
department that handles all cataloging, or for the head of a specialized cataloging unit (e.g., copy cataloging or foreign languages). List the head of library automation and computer systems, applications, programming, etc. as HDCOMP unless that person is also an Associate or Assistant Director, in which case use the appropriate administrative code. If there is an intermediate level of management between an Associate or Assistant Director and the professionals who actually carry out the analysis, programming, etc., use HDCOMP to define that intermediate level. Professionals who carry out analysis, programming, etc., should be listed as functional specialists (FSPEC).

Head, Acquisitions Department. Use HDACQ for all of the following positions: (a) head of a department that is responsible for the selection of material (or management of selection activities carried out on a basis encompassing more than a single organizational unit), but not responsible for the placement of orders, payment of invoices, etc.; (b) head of a department responsible for the placement of orders, maintaining on-order files, payment of invoices, etc., but not responsible for selection decisions; (c) head of a department responsible for both the selection decisions (or coordination of selection activities) and for acquiring the material. Libraries that split these two functions between two departments should report more than one professional with the position HDACQ.

[Special note concerning Assistant Department Heads. Assistant Department Heads who are responsible for major units and spend the bulk of their time in supervision and revision of the work of others should also be listed as "Head, Other Department/Service/Agency." See additional subcodes below for Head, Cataloging, and Head, Other Department. However, Assistant Head positions responsible for small units or for supervision only in the absence of the head should be reported as non-supervisory or specialist positions as appropriate.]

Administrative. Please note that ADMIN is not only for Administrative Services and related positions, but also can be applied to Public Relations/Communications, Development/Fundraising, and all other administrative and/or professional positions which do not have a logical home elsewhere.

5. Please indicate "Sex" with the letter M or F, indicating male or female, respectively.

6. "OEOCat" minority status code, for US university libraries only, should be indicated with one of the following code numbers. (Leave blank if a Canadian library):

$$
\begin{aligned}
& 1=\text { Black } \\
& 2=\text { Hispanic } \\
& 3=\text { Asian or Pacific Islander } \\
& 4=\text { American Indian or Native Alaskan } \\
& 5=\text { Caucasian/Other }
\end{aligned}
$$

7. "YrsExp," or total years of professional experience. For most professional staff members this will mean counting the years since the MLS degree was awarded. When counting, do not subtract interim periods when an individual was not engaged in professional library employment if these periods are short in relation to the overall professional career. Count an academic year contract period as a full year. Be sure to include professional experience in previous positions and in other institutions. The figure should be rounded off to the nearest whole number; for example, a position with 14.5 years of experience would appear as 15.

\section{Indicate "Rank" using the following system of codes:}

0 The library director. Some systems also use 0 for assistant and/or associate directors. 
1 Lowest level in the rank structure, such as an entry-level position.

2-8 Successively higher levels; for example, 5 indicates a higher rank than 2.

9 Rank cannot be determined, or, the individual is outside the organization's rank structure.

Responses concerning rank should be limited to professional librarians, and other professionals who occupy the same ranks as librarians. Leave the rank column blank for professionals who do not occupy these ranks or if the column is not applicable. For example, if the Library Business Officer holds a rank typically used for university administrators but not for librarians, do not supply a rank code for that individual, even if you have included salary and other data.

If multiple ranking structures are used for librarians and these structures are substantially different and not equivalent, enter individual rank information only for that group which represents the largest fraction of "rankand-file" librarians.

The maximum number of ranks reported here should not exceed the maximum number of rank-levels reported in Part I for individual data under Rank structure. When counting the total number of rank levels, include ranks that may be unoccupied at the present time due to circumstances like unusually high turnover, hiring freezes, etc.

9. "Percent" is used to determine if an employee works full-time or part-time. All full-time employees have Percent $=1.00$, i.e., they work $100 \%$ of a full-time schedule. If Percent is less than 1.00 , then the employee works that fraction of a full-time schedule. For example, a $65 \%$ time appointment would be entered as 0.65 . Calculate the percent appointment by dividing the amount of time an employee works by the amount considered to be the norm for full-time employment at your institution. For example, if a full-time appointment at your institution is 12 months at 40 hours per week:

○ A 9-month part-time appointment has Percent $=9 / 12$, or 0.75 .

$\circ$ An appointment at 30 hours per week has Percent $=30 / 40$, also 0.75 .

○ An appointment at 30 hours and 9 months has Percent $=0.75 \times 0.75=0.56$.

Enter Percent with two decimal points.

Optional Questions: The US Office of Management and Budget has revised the Standards for the Classification of Federal Data on Race and Ethnicity ${ }^{1}$ and according to the new standard there will be five minimum categories for data on race (American Indian or Alaska Native, Asian, Black or African American, Native Hawaiian or Other Pacific Islander, and White) and one category for data on ethnicity ("Hispanic or Latino"). Respondents will be able to report more than one race by choosing multiple responses to the race question. The purpose of the revised classification is to reflect the increasing diversity of the US population that has resulted primarily from growth in immigration and in interracial marriages. The new standards were used by the Bureau of the Census in the 2000 decennial census. In light of these developments, we are collecting the new classification on race and ethnicity in the ARL Annual Salary Survey on an optional basis.

Ethnicity should be indicated by coding 1 to indicate if the person is of Hispanic or Latino ethnicity, and coding 0 otherwise. The definition of Hispanic or Latino ethnicity is: A person of Cuban, Mexican, Puerto Rican, Cuban, South or Central American, or other Spanish culture or origin, regardless of race.

\footnotetext{
${ }^{1}$ http: / / www.whitehouse.gov/omb/bulletins/b00-02.html and http:/ / www.whitehouse.gov/OMB/ fedreg/ombdir15.html
} 
Race should be indicated for US university libraries only, by choosing one or more responses among the five racial categories provided here; $1=$ yes and $0=$ no. You can select multiple racial categories for a person. The definitions of the five racial categories, listed with their respective column names, are:

American Indian or Alaska Native (NatAm): A person having origins in any of the original peoples of North and South America (including Central America) who maintains tribal affiliation or community attachment.

Asian (Asian): A person having origins in any of the original peoples of the Far East, Southeast Asia, or the Indian subcontinent including, for example, Cambodia, China, India, Japan, Korea, Malaysia, Pakistan, the Philippine Islands, Thailand, and Vietnam.

Black or African American (Black): A person having origins in any of the black racial groups of Africa.

Native Hawaiian or Other Pacific Islander (HawPI): A person having origins in any of the original peoples of Hawaii, Guam, Samoa, or other Pacific Islands.

White (White): A person having origins in any of the original peoples of Europe, the Middle East, or North Africa.

\section{Submitting the Data for Part I and Part II on the Web}

ARL is using the online services of FormSpring to collect the data. As part of its privacy policy, FormSpring pledges not to sell any collected information to third parties. For the complete FormSpring privacy policy, visit http://www.formspring.com/privacy.html. ARL also accepts Part I and Part II of the salary survey by e-mail from those users who may be uncomfortable submitting the files in FormSpring:

- University Libraries: http://www.formspring.com/forms/?366101-c6BynijupA

- Nonuniversity Libraries: http://www.formspring.com/forms/?366133-c6BynijupA

Be sure to have the electronic copy of your completed salary survey Excel file handy as you will be submitting this file via the FormSpring Web form. In addition to the completed Excel file, be prepared to provide the following information as well:

- The name, title, email and phone number of the person who prepared the Excel file. The name, title, email and phone number of your institution's contact person for the salary survey (if different from the person who prepared the Excel file)

- Indicate whether you are submitting salary information for one or more of the following: Main, Law, or Medical library, and the beginning professional salary and rank structure for each.

For professional salary list the salary that would be paid to a newly hired professional without experience (even if local practice discourages hiring entry-level professionals without experience). Please report the 2008-2009 beginning professional salary to the best of your knowledge as it existed on July 1, 2008.

For rank structure, list the number of unique levels in your institution's rank structure. If you have no 
levels in your rank structure, use 1 . The number reported here should be equal to the highest number in the "Rank" column of your Excel file (i.e., the number of levels reported in your Excel file should equal the number of levels reported here).

- The names of the libraries that are included and excluded in your figures for the 'general libraries' (these can be main campus libraries or branch campus libraries), as well as any other explanatory information, should be indicated in a footnote. In your footnotes, report any information that would clarify the figures submitted: the inclusion and exclusion of branch campus libraries, a reporting date that is sooner/later than July 1, 2008, etc. Please make an effort to word your footnotes in a manner consistent with notes appearing in the published report, so that ARL can interpret your footnotes correctly.

Please return the completed questionnaire to the ARL Statistics and Measurement Program by September 30, 2008.

For assistance, contact Martha Kyrillidou (martha@arl.org) or Les Bland (stats@arl.org) or Kristina Justh (kristina@arl.org) or Gary Roebuck (gary@arl.org).

Tel. 202-296-2296 or fax 202-872-0884.

\section{http://www.arl.org/stats/annualsurveys/salary/}




\title{
ARL Annual Salary Survey 2008-2009 \\ UNIVERSITY LIBRARY QUESTIONNAIRE
}

\author{
Part I: Summary Data
}

Reporting Institution Date Returned to ARL

Report Prepared by (name)

Title

Email address Phone number

Contact person (if different)

Title

Email address Phone number

1. Beginning Professional Salary

Main

Law

Medical

Beginning professional salary for 2008-2009

(Note: ARL will calculate the 2008-2009 median and average professional salaries for your library from the individual data you supply in Part II of this questionnaire.)

\section{Rank Structure.}

Indicate the number of levels in your institution's rank structure for professional librarians. You should report here the maximum number of rank levels, reported in Part II for individual data, under the Rank column.

1 level (i.e., no differentiated levels)

2 levels 
3 levels

4 levels

5 levels

more than 5 levels (please specify the number of levels:

\section{FOOTNOTES}

3a. Please list which libraries are included in the data submitted for the "general" libraries. These can be main campus libraries or branch campus libraries.

3b. Please list which libraries are NOT included in the data submitted for the "general" libraries. These can be main campus libraries or branch campus libraries.

Please indicate any other explanatory information in footnotes. These additional footnotes, if necessary, should be placed in the space below or on attached pages.

Please return the completed questionnaire to the

ARL Statistics and Measurement Program by September 30, 2008.

For assistance, contact Martha Kyrillidou (martha@arl.org) or Les Bland (stats@arl.org), or call 202-296-2296 

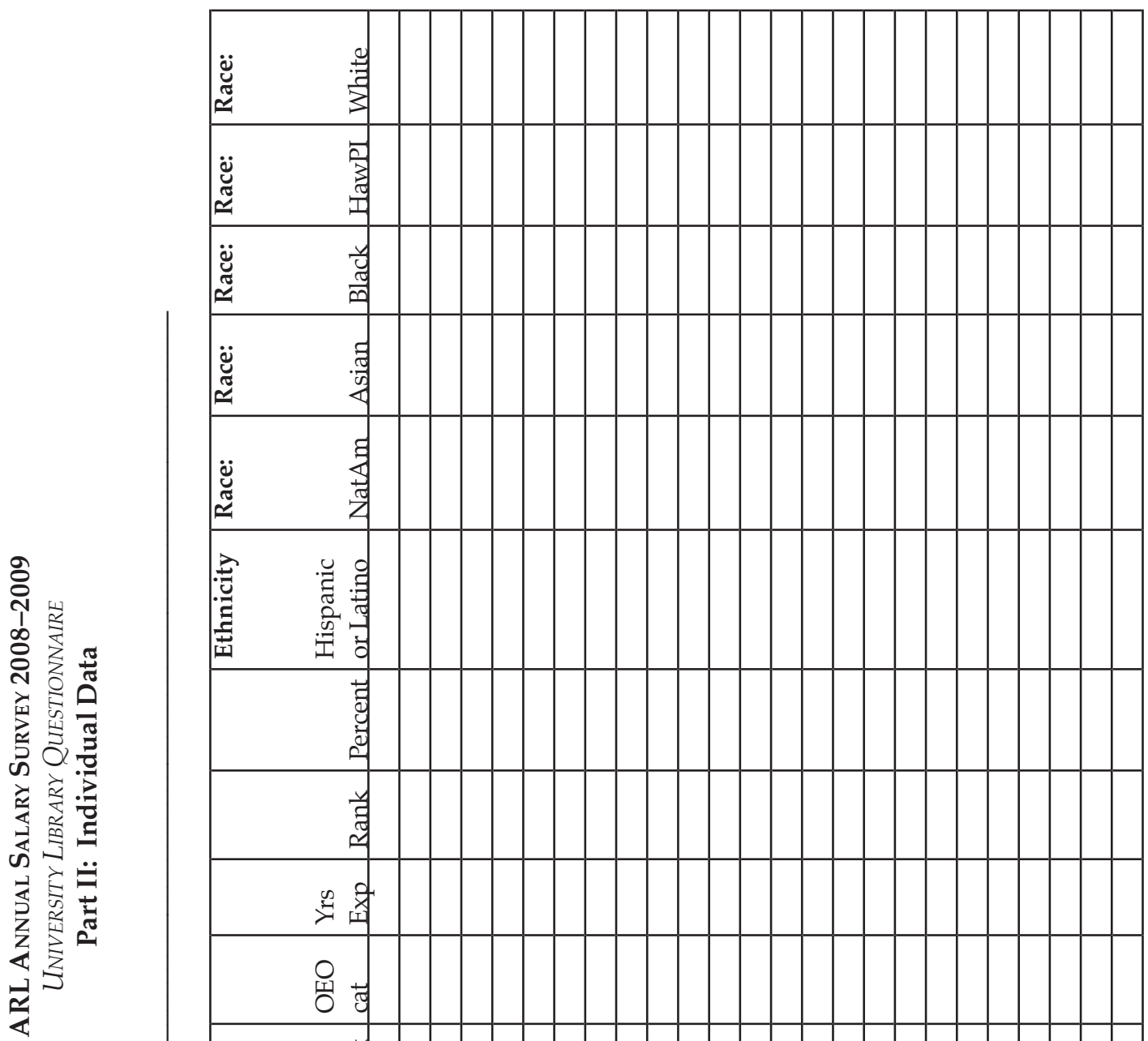

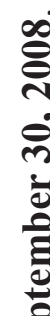
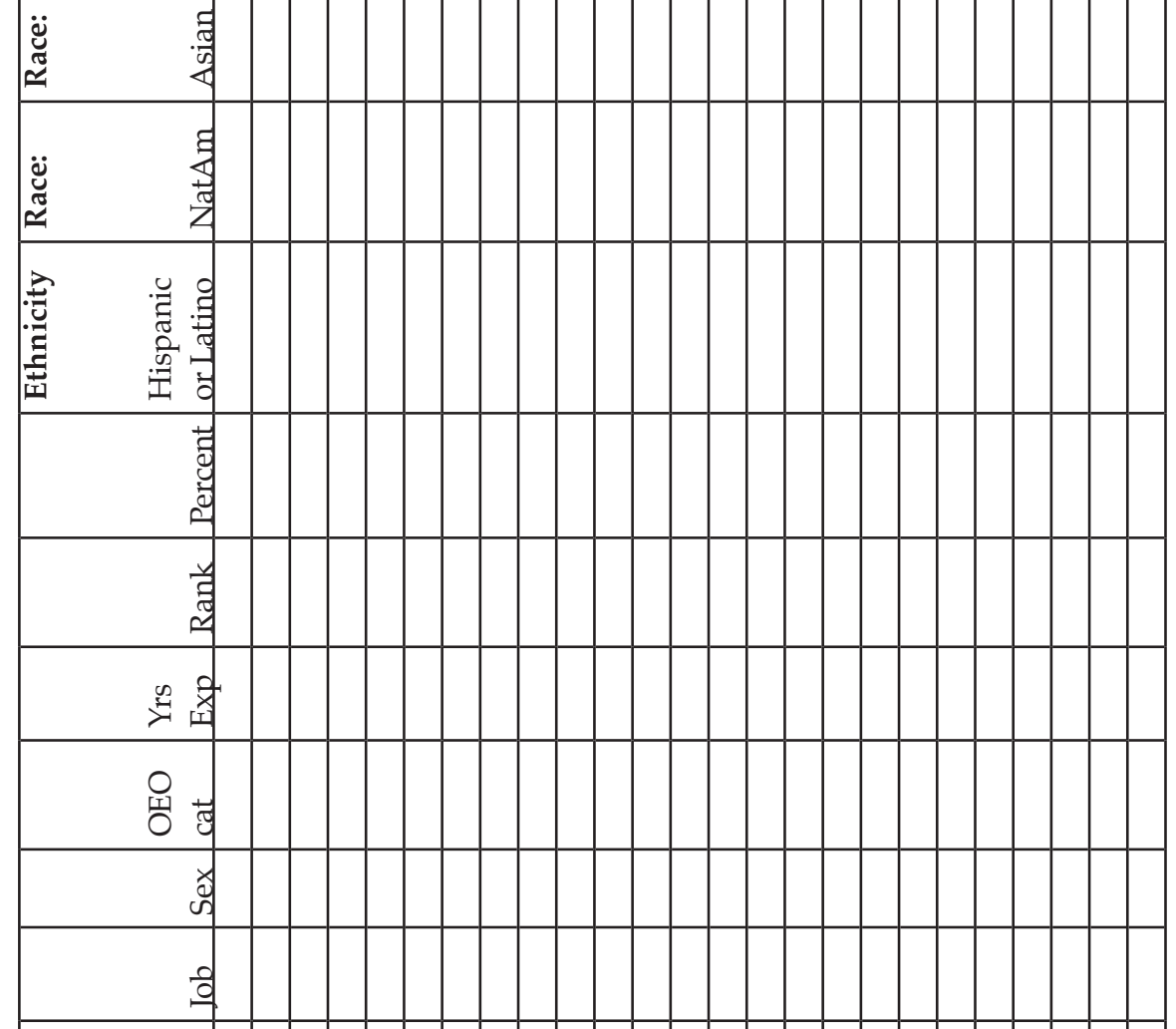

帚
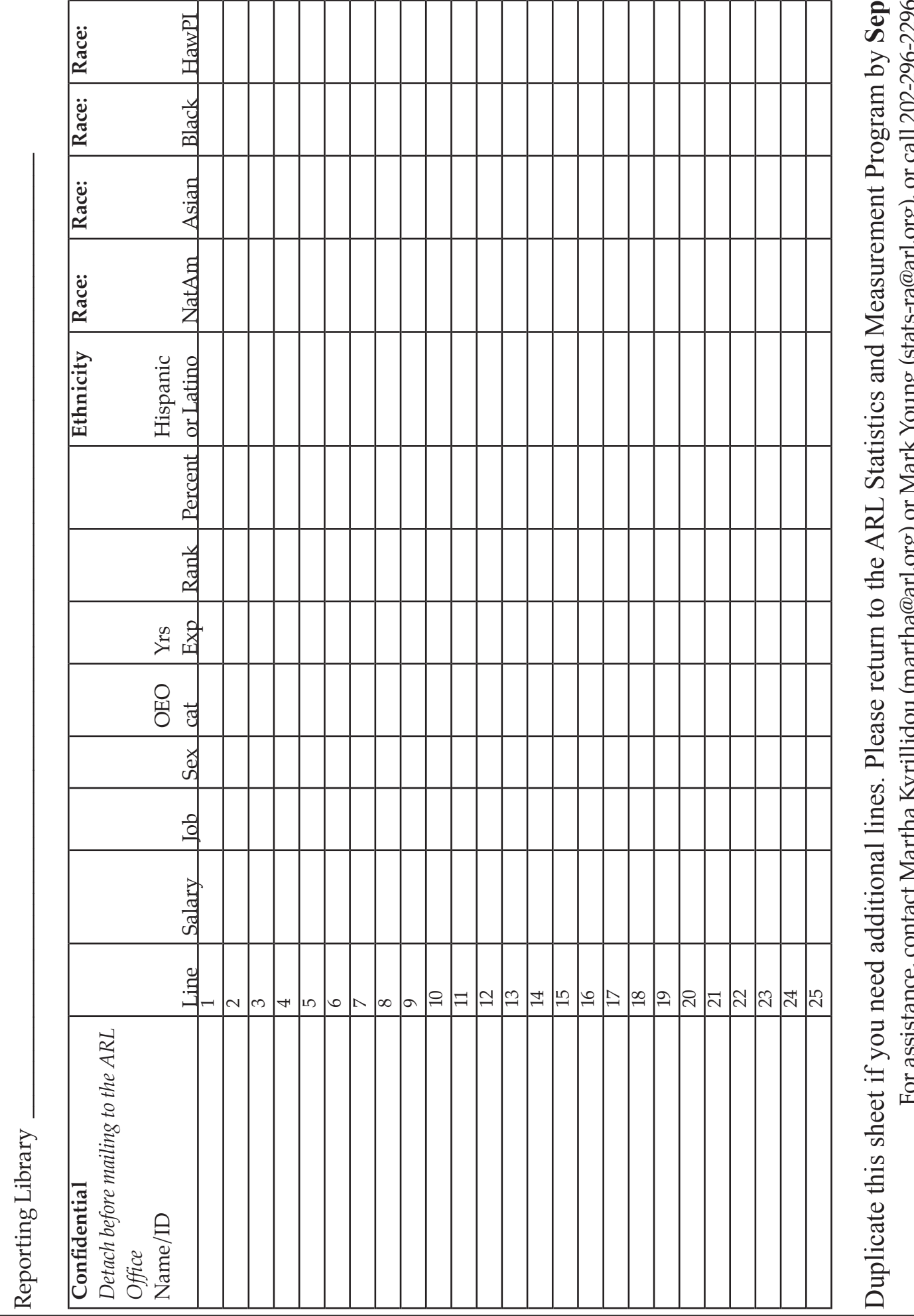
NonUniversity Library QUeSTIONNAIRE AND INSTRUCTIONS 


\title{
ARL Annual Salary Survey 2008-2009
}

\author{
NONUNIVERSITY LIBRARY QUESTIONNAIRE
}

\section{General Instructions for Completing the Questionnaire}

1. This survey is concerned with the salaries of professional positions only. Since the criteria for determining professional status vary among libraries, there is no attempt to define the term "professional." Each library should report the salaries of those staff members it considers professionals, irrespective of membership in a collective bargaining unit, and including, when appropriate, staff who are not librarians in the strict sense of the term, such as systems analysts, budget officers, etc.

2. Obtain the Word file. These instructions assume that you have Microsoft Word available for use. If not, or if you have trouble opening the files in Word, please call the ARL Statistics and Measurement Program at (202) 296-2296 or email stats@arl.org.

3. The template Word file is available at: http://www.arl.org/stats/annualsurveys/salary/salform08.shtml. This is a generic, blank form that can hold your data. The file's name is "nu_form08xxxx.doc"; open the file and save it to your own computer by choosing "Save As" under the File menu. When saving the file, please change the " $x x x x$ " in its name to your institution's name, e.g., "sal08Smithsonian.doc."

4. Salaries should be reported for all filled positions. Vacant positions should be excluded from your report.

5. Report 2008-2009 salaries as they exist on July 1, 2008. If the library normally increases salaries at a date after July 1 , and the salary as of that later date is known or can be estimated (within $\$ 100$ or so) by the time the questionnaire is due to be returned, please use the higher salary and footnote the effective date and/or whether the reported figures are known or estimated. Please do not hold up the reporting process for later salary adjustments.

6. The Median Salary is the salary that has an equal number of salaries above it and below it. In those libraries with an even number of positions, the median salary is the average of the two salaries that have an equal number of salaries above and below them.

7. The Beginning Professional Salary is the salary that would be paid to a professional without experience, not necessarily the lowest professional salary paid. In reporting the beginning salary, please use a figure that is actually used or likely to be used for entry-level librarians hired by your library.

8. Salaries should be reported for both full-time and part-time professional positions. However, salaries for part-time positions should be converted to their full-time equivalents before reporting; do not report the actual part-time salary paid.

9. Salaries should normally be reported on a 12-month basis. If an appointment is for 9 or 10 months at the option of the employee, the actual salary paid should be increased to its 12 -month equivalent. However, if appointments of less than 12 months are required by the employer, report the actual salary paid.

10. The salaries for all professional positions should be included, regardless of whether the salaries come from regular library budget funds or from special funds such as research grants. 
11. The salary figures should be straight gross salary figures. Do not include fringe benefits.

12. Explanatory footnotes to the reported figures may be provided when necessary. Footnotes will be included in the published survey.

13. Provide the name of the reporting library and the name of the person who prepares the report.

14. Use the newly available Web form for your data submission:

(http://www.formspring.com/forms/?366133-c6BynijupA). Fill in Part I on the Web and upload your file for Part II through the same interface. NOTE: You must complete the entire submission in a single session. The Web interface does NOT allow you to return and edit your information once it is submitted.

Note: ARL is using the online services of FormSpring to collect the data. As part of its privacy policy, FormSpring pledges not to sell any collected information to third parties. For the complete FormSpring privacy policy, visit http://www.formspring.com/privacy.html. ARL also accepts Part I and Part II of the salary survey by e-mail attachment from those users who may be uncomfortable submitting the files in FormSpring.

Please Submit the Web form by September 30, 2008.

For assistance, contact Martha Kyrillidou (martha@arl.org) or Les Bland (stats@arl.org) or Kristina Justh (kristina@arl.org) or Gary Roebuck (gary@arl.org). Tel. 202-296-2296 or fax 202-872-0884. 


\section{ARL Annual Salary Survey 2008-2009 \\ NONUNIVERSITY LIBRARY QUESTIONNAIRE}

\section{Part I: Summary Data}

Reporting Institution Date Returned to ARL

Report Prepared by (name)

Title

Email address Phone number

Contact person (if different)

Title

Email address Phone number

1. Complete the table on the back of this sheet by indicating the number of filled or temporarily vacant professional positions in each salary range for fiscal years 2007-2008 and 2008-2009.

2. Median professional salary for fiscal year 2008-2009:

3. Beginning professional salary for 2008-2009:

4. Footnotes (please compare with footnotes from surveys of previous years)

a. Law Library salaries are included.

Yes

No

We do not have a Law Library.

b. Medical Library salaries are included.

Yes

No

We do not have a Medical Library.

c. Branch libraries not included (please attach an additional sheet if necessary):

5. Other comments (please attach an additional sheet if necessary): 


\section{Part II Salaries:}

Indicate the number of filled professional positions in each salary range for fiscal years 2007-2008 and 2008-2009.

\begin{tabular}{|c|c|c|}
\hline & \multicolumn{2}{|c|}{ Number of Positions } \\
\hline Salary Range & $2007-2008$ & 2008-2009 \\
\hline \multicolumn{3}{|l|}{ More than 250,000 } \\
\hline \multicolumn{3}{|l|}{$200,000-250,000$} \\
\hline \multicolumn{3}{|l|}{$175,000-199,999$} \\
\hline \multicolumn{3}{|l|}{$150,000-174,999$} \\
\hline \multicolumn{3}{|l|}{$140,000-149,999$} \\
\hline \multicolumn{3}{|l|}{$130,000-139,999$} \\
\hline \multicolumn{3}{|l|}{$120,000-129,999$} \\
\hline \multicolumn{3}{|l|}{$110,000-119,999$} \\
\hline \multicolumn{3}{|l|}{$100,000-109,999$} \\
\hline \multicolumn{3}{|l|}{$95,000-99,999$} \\
\hline \multicolumn{3}{|l|}{$90,000-94,999$} \\
\hline \multicolumn{3}{|l|}{$85,000-89,999$} \\
\hline \multicolumn{3}{|l|}{$80,000-84,999$} \\
\hline \multicolumn{3}{|l|}{$76,000-79,999$} \\
\hline \multicolumn{3}{|l|}{$74,000-75,999$} \\
\hline \multicolumn{3}{|l|}{$72,000-73,999$} \\
\hline \multicolumn{3}{|l|}{$70,000-71,999$} \\
\hline \multicolumn{3}{|l|}{$68,000-69,999$} \\
\hline \multicolumn{3}{|l|}{$66,000-67,999$} \\
\hline \multicolumn{3}{|l|}{$64,000-65,999$} \\
\hline \multicolumn{3}{|l|}{$62,000-63,999$} \\
\hline \multicolumn{3}{|l|}{$60,000-61,999$} \\
\hline \multicolumn{3}{|l|}{$58,000-59,999$} \\
\hline \multicolumn{3}{|l|}{$56,000-57,999$} \\
\hline \multicolumn{3}{|l|}{$54,000-55,999$} \\
\hline \multicolumn{3}{|l|}{$52,000-53,999$} \\
\hline $50,000-51,999$ & & \\
\hline $48,000-49,999$ & & \\
\hline $46,000-47,999$ & & \\
\hline $44,000-45,999$ & & \\
\hline $42,000-43,999$ & & \\
\hline $40,000-41,999$ & & \\
\hline $38,000-39,999$ & & \\
\hline $36,000-37,999$ & & \\
\hline $34,000-35,999$ & & \\
\hline $32,000-33,999$ & & \\
\hline $30,000-31,999$ & & \\
\hline less than 30,000 & & \\
\hline Total Number of $\mathrm{Po}$ & & \\
\hline
\end{tabular}

Please submit the completed questionnaire to the web form at: http://www.formspring.com/forms/?366133-c6BynijupA

by September 30, 2008. For assistance, contact Martha Kyrillidou (martha@arl.org) or Les Bland (stats@arl.org) or Kristina Justh (kristina@arl.org) or Gary Roebuck (gary@arl.org). Tel. 202-296-2296 or fax 202-872-0884. 



\section{Footnotes to the ARL Annual Salary Survey, 2008-2009}

INSTITUTION

ALABAMA

ALBERTA

ARIZONA

ARIZONA STATE

\section{AUBURN}

BOSTON COLLEGE

BRIGHAM YOUNG

BRITISH COLUMBIA
Note

Includes the Amelia Gayle Gorgas Library, Rodgers Library, Bruno Business Library, Hoole Special Collections Library, McClure Education Library.

Excludes the Bounds Law Library, Health Sciences Library.

Includes the following libraries: Bibliographic Services, HT Coutts Education Library, Humanities \& Social Sciences Library, Faculty Saint-Jean Library, Winspear Business Reference Library, Office of Staff Development \& Training, Cameron Library (including: Interlibrary Loans/Document Delivery, Financial Systems \& Analysis, Science \& Technology Library, Information Technology Resource Services).

Excludes the John W. Scott Health Sciences Library and the J.A. Weir Memorial Law Library.

Includes the following libraries: 1) Main Library, 2) The Center for Creative Photography, 3) Fine Arts Library, 4) Science-Engineering Library, 5) Special Collections.

Includes data for Arizona Health Sciences Library from both Tucson (main) and Phoenix (branch) campuses.

Includes: ASU Tempe Campus Libraries, ASU-West Campus Library,ASU- Polytechnic Campus Library, ASU-Downtown Phoenix Campus Library, and the ASU Law Library. Includes the Main Campus Library.

Includes the O'Neill Library (main library), Theology and Ministry Library, Bapst Art Library, Educational Resource Center, Social Work Library, and the Law Library.

Includes the main campus library.

Excludes the Hunter Law Library.

The libraries included are: Art-Architecture-Planning, Asian Library, David Lam Management Library, Education Library, Irving K. Barber Learning Centre (previously in Main Library), Koerner Library (Humanities \& Social Sciences, Borrower Services), Law Library, Library Processing Centre (Technical services and Systems). Also includes the Life Science Libraries: Biomedical Branch Library, Hamber Library, St. Paul's Hospital Library, Woodward Biomedical Library, Music Library, the Okanagan Library, Rare Books and Special Collections Division, Robson Square Library, Science and Engineering Division, Xwi7xwa Library (First Nations House of Learning).

Excluded: reading rooms and affiliated libraries.

The University of British Columbia Library system has no rank structure.

Includes the following libraries: Rockefeller Library, John Hay Library, Orwig Music Library, Art/Slide Library, Sciences Library, John Carter Brown Library. 


\section{INSTITUTION}

\section{CALIFORNIA, BERKELEY}

\section{CALIFORNIA, DAVIS}

\section{CALIFORNIA, LOS ANGELES}

\section{Note}

Includes the General Library: Doe, Moffitt, Bancroft, Anthropology, Art History/ Classics, Astronomy- Mathematics- Statistics, Bioscience and Natural Resources, Business \& Economics, Chemistry, C.V. Starr East Asian Library (including Center for Chinese Studies), Earth Sciences, Education- Psychology, Engineering, Environmental Design, Music, Optometry, Physics, Public Health (including Health Sciences Information Services, and Occupational \& Environmental Health), and Social Welfare libraries, and the Northern Regional Library Facility.

Excludes Affiliated Libraries: Architectural Slide, Continuing Education of the Bar, Earthquake Engineering, Ethnic Studies, Giannini, Institute of Governmental Studies, Institute of Industrial Relations, Institute of International Studies, Institute of Transportation Studies, and Water Resources Center libraries. Also excluded are the Law Library and various departmental libraries: e.g., French, History, Philosophy, Rhetoric, and Slavic Languages and Literature.

Beginning 2004-2005, UCB salary figures include administrative stipends, where applicable.

Includes the Peter J. Shields Library (Davis Campus); Physical Sciences \& Engineering Library (Davis Campus); the Carlson Health Sciences Library (Davis Campus); the Medical Center Library (Sacramento Campus), and the Argicultural \& Resource Economics Library (Davis Campus).

Librarians who are department heads have received administrative stipends since July 1, 1999, but these stipends were not included in the salaries reported to ARL until the salary survey of 2004-2005. We continue to include these stipends when providing salary information for our department heads.

Includes the Arts Library, College Library (Undergraduate Library), Eugene and Maxine Rosenfeld Management Library, Music Library, Richard C. Rudolph East Asian Library, Science \& Engineering Library, Social Sciences and Humanities Library (Charles E. Young Research Library), and the Southern Regional Library Facility.

Includes data for 12 affiliated libraries on the UCLA campus including: 1) American Indian Studies Center; 2) Ralph M. Bunche African-American Studies Center; 3) Asian American Studies Center; 4) Chicano Studies Research Center; 5) Ethnomusicology Archive; 6) Film \& Television Archive; 7) Graduate School of Education \& Information Studies, Department of Information Studies; 8) Institute for Social Science Research; 9) Latin American Center/Hispanic American Periodicals Index; 10) Olive View Medical Center; 11) Grace M. Hunt English Reading Room; 12) William Andrews Clark Memorial Library.

Librarians who are department heads have received administrative stipends since January 1998; however, these stipends were not included in the salaries reported to ARL prior to 2003. Beginning with the 2003 survey, UCLA now includes those stipends in salaries reported for department heads. Interim department heads also receive stipends and these are reported in the survey.

The General Library Survey includes three Council of Library \& Information Resources (CLIR) Fellows.

The beginning professional salary increased to \$46,164 on April 1, 2008. 


\section{INSTITUTION}

CALIFORNIA, LOS ANGELES, cont.

\section{CALIFORNIA, RIVERSIDE}

CALIFORNIA, SAN DIEGO

CALIFORNIA, SANTA BARBARA

CANADA INSTITUTE FOR SCIENTIFIC AND TECHNICAL INFORMATION

CASE WESTERN RESERVE

CENTER FOR RESEARCH LIBRARIES

CHICAGO

CINCINNATI

COLORADO

COLORADO STATE

CONNECTICUT

CORNELL

DARTMOUTH

DUKE

EMORY

\section{Note}

In the Medical Library survey, job categories have been updated to reflect a change in organizational structure and the addition of two associate director positions (these were previously department head positions).

Includes the Rivera Library (serving the College of Humanities, Arts, and Social Sciences, School of Education, and the Graduate School of Management); Science Library (serving the College of Natural \& Agricultural Sciences \& the College of Engineering); and the Palm Desert Campus Library (serving the Graduate School of Management \& the Graduate Division).

Excludes the Media and Music Libraries: (there are no professional librarians in these facilities).

Includes the following libraries: Social Sciences \& Humanities, Science \& Engineering, Special Collections, ARTS, International Relations \& Pacific Studies, Center for Library \& Instructional Computing Services, Scripps Institution of Oceanography.

Includes the Main Library and Arts Library.

All branch libraries are included and all values were reported in Canadian dollars.

Includes the Kelvin Smith Library (main library), Harris Library of the Mandel School of Applied Social Sciences and the Kulas Music Library.

Survey size dropped from 31 personnel in 2007-2008 to 29 in this reporting period. This was caused by a new determination concerning the definition of "professional" in the center's staff.

All libraries included in main and well as law and medicine.

All main campus libraries are included and branch campus libraries are excluded. Included: Norlin, Music, Business, Math/Physics, Engineering, and Earth Sciences Libraries.

All main campus and branch campus libraries are included.

Includes the following libraries: Homer Babbidge Library, Dodd Research Center, Stamford Campus Library, Torrington Campus Library, Waterbury Campus Library, Avery Point Campus Library, Greater Hartford Campus Library. Both the University of Connecticut Law Library and University of Connecticut Health Sciences Library are also included.

The following libraries are included: Africana, Engineering, Entomology, Fine Arts, Geneva Experiment Station, Hotel Administration, Management, Mann Library, Math, Music, ILR, Olin/Kroch/Uris, Physical Science, Veterinary Medicine. Both the Cornell Medical Library and Cornell Law Library are included.

Includes: Baker-Berry Library, Paddock Music Library, Sherman Art Library, Kresge Physical Sciences Library, Feldberg Business \& Engineering Library, Rauner Special Collections Library, and the Storage Library.

Duke University Medical Center Library, Perkins Library System, Divinity School Library, and the Law Library are included.

Data submitted for the "general" libraries includes: General Libraries, Theology Library, and the Oxford College Library. 


\section{INSTITUTION \\ FLORIDA}

FLORIDA STATE

\section{GEORGETOWN}

GEORGIA

GEORGIA TECH

GUELPH

\section{HARVARD}

\section{HAWAII}

\section{HOUSTON}

\section{HOWARD}

\section{ILLINOIS, CHICAGO}

\section{Note}

Included: Humanities and Social Sciences Library, Science Library, Journalism Library, Music Library, Education Library, Architecture and Fine Arts Library, Health Science Center Library and the Legal Information Center.

Includes: Strozier Library (Main); Dirac Science Library; Allen Music Library; Goldstein College of Information Library; Panama City, Florida Branch Library; Ringling Museum Library; and the Career Center Library. Law and health libraries are also included.

Includes: Woodstock Theological Library, Blommer Science Library, Lauinger Library, Law Library, Medical Library.

The Bioethics Library is included with the Main Library.

Main Library, Science Library, Map Library, Student Learning Center Library, Curriculum Learning Center Library, several reading rooms and experiment station libraries located around the State of Georgia. The Law Library is also included.

The Main Library and the Architecture Library are included.

Main Campus Libraries included: McLaughlin Library and the OVC Learning Commons Library. Branch Campus Libraries included: Guelph-Humber Learning Commons Library and the Ridgetown Campus Library.

All salary values were reported in Canadian Dollars (\$CAD).

Individual rank data have been included for professional librarians with only the Library Director assigned a rank of 0 . Non-librarian professionals are assigned rank of 9 . Includes all libraries on the Boston and Cambridge campuses, including the Schlesinger Library at Radcliffe College.

Excludes Biblioteca Berenson (Florence, Italy), Dunbarton Oaks Research Libraries (Washington, DC), and the Center for Hellenic Studies (Washington, DC).

The salaries for Harvard Medical and Harvard Law libraries do not include compensation for the research and professorial duties of this position, yet they are considered full time.

The law library is not a branch of the main university library. It is independent, with a separate director, who reports to the dean of the law school.

Includes: Architecture Library, Music Library, Weston A. Pettey Optometry Library, and the Pharmacy Library.

Includes law library, main library, and the Louis Stokes Health Sciences Library. Moorland-Springarn Research center is also incorporated.

The Louis Stokes Health Sciences Library (LSHSL) is independent from the general libraries and is not a branch library.

The University of Illinois, Chicago is one campus (including Peoria, Rockford, Urbana).

Part time salaries listed at actual part time rate (rather than annualized) per ARL instructions.

Job categories: HDBR is used for heads of branch sites (as well as the Science Library) HDOTH is used for heads of internal departments not listed in ARL categories, such as GMRMLN. FSPEC denotes all Systems Librarians. 


\author{
INSTITUTION \\ ILLINOIS, CHICAGO, cont. \\ ILLINOIS, URBANA \\ INDIANA
}

IOWA

IOWA STATE

JOHNS HOPKINS

KENT STATE

KENTUCKY

LAVAL

LIBRARY OF CONGRESS LOUISIANA STATE

LOUISVILLE

MANITOBA

MASSACHUSETTS
Note

Ethnicity and race information was gathered from a mailing to academic staff and from information supplied on new employee NETID request form.

Includes main campus libraries and law library.

Main campus libraries and the Indianapolis School of Law are included.

Excludes: Dentistry Library; Medicine Library; IUPUI University Library; Herron School of Art Library; Columbus Library; Science \& Engineering Library; and other campuses libraries at IU-East; IU-Kokomo, IU-Northwest, IU-Southeast, IU-South Bend, and IPFW-Fort Wayne.

Includes the main library and its ten branches. The Law and Medical Libraries are also reported.

Includes the Parks Library (main library) and the Veterinary Medical Library.

Iowa State has five ranks: $1=$ assistant professor, $2=$ associate professor, $3=$ professor, $0=$ dean or associate dean, 9=Professional \& Scientific (a non-faculty classification).

Includes the Sheridan Libraries, Friedheim Library, and the School of Advanced International Studies Library.

Includes libraries on the following campuses: Kent, Ashtabula, East Liverpool, Geauga, Salem, Stark, Trumbull, and Tuscarawas.

Includes the William T. Young Library (Main campus Library), Agricultural Information Center, Chemistry/Physics Library, Design Library, Education Library, Engineering Library, Medical Center Library, Law Library, Special Collections \& Digital Programs, Little Fine Arts Library, Equine Library, Geological Science Library and Maps Collection, and the Kentucky Transportation Center.

All libraries are included.

This report includes two people who began employment in January 2009.

Salaries include professional and administrative positions.

The Law School Library and Veterinary Medicine Library are included.

While geographically in the same location (Baton Rouge), the Law School and the School of Veterinary Medicine are considered separate campuses.

Included: Main library, Music library, Art library, and University Archives.

The following are included: William R. Newman Library, Architecture/Fine Arts Library, Elizabeth Dafoe Library, Fr. Harold Drake Library, St. John's College Library, Donald W. Craik Engineering Library, E.K. Williams Law Library, Adlbert D. Cohen Management Library, Eckhardt-Gramatte Music Library, Sciences and Technology Library, Neil John Maclean Health Sciences Library, Bill Larson Library, Carolyn Sifton-Helene Fuld Lilbrary, Concordia Hospital Library, J.W. Crane Memorial Library, Misericordia Health Centre Library, Riverview Health Centre Library, Seven Oaks General Hospital Library, and the Victoria General Hospital Library.

The DuBois, Integrated Sciences \& Engineering, and Image Collection Libraries are included. 


\section{INSTITUTION \\ MICHIGAN}

\section{MICHIGAN STATE}

MISSOURI

MONTREAL

NATIONAL AGRICULTURAL LIBRARY NATIONAL LIBRARY OF MEDICINE NEBRASKA

\section{NEW MEXICO}

\section{NEW YORK}

\section{NORTH CAROLINA \\ NORTH CAROLINA STATE}

NORTHWESTERN

\section{Note}

The following libraries are included: Harlan, Hatcher Graduate, Music, Social Work, Askwith Media, Fine Arts, Art-Architecture-Engineering, Shapiro Science, Map, Special Collections, Shapiro Undergraduate, Asia, Public Health Science, Public Health, Museums, Area Programs, Biological Sciences, Government Documents, Papyrology, and Law.

Excludes the following libraries: Kresge Business, Clements Historical, Bentley and Gerald R. Ford Presidential.

Includes all main campus libraries.

Excludes the Law library.

Includes the Math Library, Engineering Library, Geology Library, Journalism Library, and the Vet/Med Library.

Libraries included: Environmental Development, Library and Information Sciences, Botany, Chemistry, Educational Resources, Education-Communication-PsychologyPsychoeducation-Biology, Geography, Kinesiology, Humanities and Social Sciences, Rare books and Special Collections, Mathematics and Computer Sciences, Veterinary, Music, Optometry, Physics, Law, Health and Paramedics.

All salaries are tracked with benefits.

The federal fiscal year runs from 1 October to September 30.

Included: Don Love Library (Main), C. Y. Thompson Library, Geology Library, Mathematics Library, Architecture Library, Engineering Library, Schmid Law Library, and the McGoogan Library of Medicine.

The following libraries are included: Centennial Science and Engineering Library, Fine Arts and Design Library, Parish Memorial Library, and the Zimmerman Library.

Not included: Gallup Branch Campus Library, Los Alamos Branch Campus Library, Taos Branch Campus Library, Valencia Branch Campus Library.

Two vacant Associate Director positions in the Medical library are not included for 2008-2009. An Executive Project Director position has ended. New positions included: Information Services Librarian, Archivist, Multimedia Development Specialist, and Health Insurance Portability and Accountability Act (HIPAA) Privacy Officer. Job Codes have been updated to properly reflect organizational structure.

Elmer Holmes Bobst Library, Institute of Fine Arts Library, Courant Institute of Mathematical Sciences Library, Real Estate Library, Institute for Studies of the Ancient World Library, Law Library, and the Medical School Library are included in this survey. The Library at the Polytechnic Institute of New York is not included.

New York University uses a three-rank structure: 1= Library Associate, 2= Assistant Curator, $3=$ Associate Curator.

Includes main campus and branch libraries.

Includes D. H. Hill (main) Library, Design Library, Natural Resources Library, Textiles Library, Veterinary Medical Library, and the College of Education Media Center.

Includes main, law, and the Galter Health Sciences Library. 


\section{INSTITUTION \\ NOTRE DAME}

OHIO

OHIO STATE

\section{OKLAHOMA}

\section{OKLAHOMA STATE}

\section{OREGON}

PENNSYLVANIA

PENNSYLVANIA STATE

\section{PITTSBURGH}

PRINCETON

\section{Note}

Hesburgh Libraries include the main library as well as the Chemistry/Physics, Kellogg/ Kroc Information Center, Fine Arts/Architecture, Art Slide, Business Information Center, Life Sciences, Mathematics, and Engineering Libraries.

Includes the main campus (Main library plus Music/Dance Library) and five Regional Campus libraries (Eastern, Southern, Chillicothe, Lancaster, and Zanesville).

General increase in librarian salaries due to campus market equity infusion of $\$ 90,000$.

Six additional branch campus librarians were included that had not been counted in previous surveys.

One position was abolished.

Eight positions were reclassified and are no longer counted as professional.

The Dean's vacancy from 2007-2008 was filled.

The librarian rank structures were re-evaluated and removed.

Included: main campus libraries, regional campus libraries, and libraries of the Agricultural Technical Institute and the Ohio Agriculture Research and Development Center.

Several specialized departmental research libraries on the main campus which are not part of the University Libraries system were not included in this report.

Salary data are as of 1 October 2008.

Included: Architecture Library, Chemistry/Math Library, Engineering Library, Fine Arts Library, Geology Library, Physics/Astronomy Library.

The following are included: Main Library, Veterinary Medicine Library, Architecture, Curriculum Materials, OSU Health Sciences Center Library, OSU-Oklahoma City, OSUTulsa, OSU-Okmulgee.

Included: Knight Library (main library), Architecture and Allied Arts Library, Science and Math Libraries, Portland Library and Learning Commons, Oregon Institute of Marine Biology, and the Law Library.

Includes the Museum Library, Fine Arts Library, Physical Sciences Libraries, Business Library, Center for Advanced Judaic Studies Library, Rare Book \& Manuscript Library, and Music Library.

The Communication Library is not included.

All the libraries at the University Park main campus are included. Libraries at the following branches: Abington, Altoona, Beaver, Berks, Brandywine, DuBois, Erie, Fayette, Great Valley, Greater Allegheny, Harrisburg, Hazleton, Lehigh Valley, Mont Alto, New Kensington, Shenango, Schuylkill, Wilkes-Barre, Worthington-Scranton, and York are also included.

Includes the University Library System; excludes the law library and excludes branches campuses at Titusville, Johnstown, Bradford, and Greensburg.

Includes the Firestone Library, Marquand Library of Art \& Archaeology, Mendel Music Library, Architecture Library, Stokes Library, East Asian Library, Engineering Library, Lewis Library, and Cotsen Children's Library. 


\section{INSTITUTION \\ PRINCETON, cont.}

\section{PURDUE}

QUEEN'S

ROCHESTER

RUTGERS

SASKATCHEWAN

SOUTH CAROLINA

SOUTHERN CALIFORNIA

SUNY-ALBANY

SUNY-BUFFALO

SUNY-STONY BROOK

SYRACUSE

TEMPLE
Note

The new Lewis Library includes the following libraries: Science and Technology, Biology \& Life Sciences, Chemistry, Astrophysics, Physics, Math, and Geosciences. Includes the library system on the West Lafayette campus, consisting of 11 subject libraries, an undergraduate library, and an archives and special collections unit. Excludes libraries at the regional campuses: Purdue North Central (Westville), Purdue Calumet (Hammond), and Indiana University-Purdue University, Fort Wayne. Includes the following libraries: Stauffer, Humanities and Social Sciences, Douglas, Engineering and Science, W. D. Jordan, Music and Special Collections, Education Library, Central Technical Services, Law, and Health Sciences.

Includes River Campus Libraries, Edward G. Miner Medical Library \& Sibley Music Library.

Includes the Newark Law Campus, Camden Law Campus, Research and Instructional Services, John Cotton Dana Library, Paul Robeson Library, Technical and Automated Services, Alexander Library, Mabel Smith Douglas Library, Kilmer Library, and the Library of Science and Medicine (and branches).

Excludes the School for Management and Labor Relations, and the Center for Alcohol Studies.

Besides the main library, branches that are included are: Natural Sciences, Veternary/ Medical, Education, and Engineering.

Number of employees reported is based on those working in the library as of July 1 , 2008.

Includes the Thomas Cooper Library, South Caroliniana Library, Business Library, Music Library, Math Library, Film Library, South Carolina Political Collections, Law Library, and the School of Medicine Library.

The following libraries are included: Accounting, Architecture \& Fine Arts, Boeckmann Center, Business, Cinematic Arts, Doheny, East Asian, Feuchtwanger Memorial,

Gerontology, Grand Avenue, Leavey, Music, Philosophy, Science \& Engineering, Special Collections, University Archives, and the Von Kleinschmidt Center.

Includes all main campus and branch libraries.

Salaries include administrative stipends and temporary hires. Report does not include classified staff, employees at SL-2 level or discretionary increases.

Includes Arts \& Sciences Libraries, Music Library, Special Collections (Archives, Poetry, and Rare Books).

Excludes temporary hires, classified staff, and employees at the SL-2 salary level.

Includes main campus, Health Sciences Library, and all branch libraries.

Includes the main campus library, Science \& Technology Library, Geology Library, Math Library, and the Law Library.

Does not include the Medical Library and college reading rooms.

Libraries which are included in the data submitted for the "general" libraries: Paley Library; Science, Engineering \& Architecture Library (SEAL); Ambler Library; and the Tyler School of Art Library. 


\section{INSTITUTION \\ TEMPLE, cont.}

TENNESSEE

TEXAS

TEXAS A\&M

TEXAS TECH

\section{TORONTO}

UTAH

VANDERBILT

VIRGINIA

WASHINGTON
Note

General libraries: Minimum beginning professional salary of $\$ 42,744$ is based on an 11 -month contract. Minimum beginning salary for a 10 -month contract is $\$ 39,000$.

As of data submission, there were no across-the-board increases on July 1, 2008.

Main, Law, Knoxville Medical, and Memphis Medical libraries are included.

The medical libraries have a different starting salary and rank structure from the main library. The Knoxville Medical Library starting salary is $\$ 40,000$ and has a two- level rank structure. The Memphis Medical Library starting salary is $\$ 45,000$ and has a fivelevel rank structure.

Includes the Center for American History, the Harry Ransom Humanities Research Center, Tarlton Law Library, and the University of Texas Libraries.

The following libraries are included: Sterling C. Evans Library, Cushming Memorial Library, West Campus Library, Medical Sciences Library, Policy Sciences and Economics Library, Jack K. Williams Library (Galveston Campus), Texas A\&M University at Qatar Library (Qatar Campus).

Due to Hurricane Ike, data current data for Galveston, TX's Jack K. Williams Library could not be collected. In lieu of this, data from the previous fiscal year was submitted. Survey size for main library has increased from 98 in 2007-2008 to 138 in this reporting period (2008-2009). This was caused by: reporting the medical library as part of main (a separate medical salary survey was not submitted); a new determination concerning the definition of "professional" in the TAMU libraries; and an aggressive hiring strategy to increase professional staff in order to reduce student-to-professor ratio.

Includes the University Library, Southwest Collections/Special Collections Library, Law Library, Health Sciences Library, and the Architecture library.

The Health Sciences Center reports five levels in its rank structure.

The Law Library reports four levels in its rank structure.

Salary data are as of September 1, 2009.

The following libraries are included: Main, Nadine, Vorhoff, Turchin Business Library, Law, and Medical.

Includes the Main Library, Eccles Health Sciences Library, and the Quinney Law Library.

The data submitted includes: the Central Library; Divinity Library; Peabody Library; Management Library; Science and Engineering Libraries; Special Collections and University Archives; centralized Technical Service Facility; Library Information Technology Service; University Library Administration; and the TV News Archive. Includes the following University of Virginia Libraries: Alderman (Main), Astronomy, Biology/Psychology, Chemistry, Clemons (Undergraduate), Education, Fiske Kimball Fine Arts, Math, Music, Physics, Brown Science/Engineering, Small Special Collections, Darden Graduate Business, Claude Moore Health Sciences, and Authur Morris Law. Excludes the University of Virginia College branch campus at Wise.

Includes librarians on the Seattle, Bothell \& Tacoma campuses of the University of Washington. 


\section{INSTITUTION \\ WASHINGTON STATE}

WASHINGTON U. - St. LOUIS

WATERLOO

WAYNE STATE

WESTERN ONTARIO

\section{WISCONSIN}

YALE
Note

Includes all main campus libraries and the following branches: WSU-Riverpoint Campus, WSU-Tri Cities, WSU-Vancouver, and the WSU Energy Library.

Includes the Central Library and departmental libraries in Art \& Architecture, Biology, Business, Chemistry, Earth \& Planetary Sciences, East Asian, Music, Physics, and Social Work. The Law and Medical libraries are also included.

Includes the following libraries: Dana Porter Library, Davis Centre Library, University Map Library, and the Musagetes Architecture Library.

Includes all main and extension center campuses. Law and Medical Libraries are also included.

Includes: the C. B. "Bud" Johnston Library (Business), Education Library, Music Library, Allyn and Betty Taylor Library (Health Sciences, Medicine, Dentistry, Nursing), The D.B. Weldon Library (Arts and Humanities, Social Sciences, Library Sciences, Journalism), and the Western Archives.

Excludes Affiliated University College Libraries: Brescia, Huron, King's, and St. Peter's Seminary Library.

The following libraries are included: Memorial, Special Collections, Archives, Music, Art, Geography, Geology, Steenbock Agriculture and Life Science, College, Chemistry, Math, Business, Social Work, Social Science Reading Room, Wendt Engineering, Physics, Biology, Law, Ebling (Health).

Excluded: Wisconsin Historical Society, Center for Instructional Media, SLIS, Robinson Map Library, and the Primate Center Library.

Includes: the African Collection, Anthropology Library, Area Studies Library, Arts Library, Arts of the Book Collection, Astronomy Library, Babylonian Collection, Bass Library, Beinecke Rare Book and Manuscript Library, Center for British Art, Chemistry Library, Classics Library, Divinity School Library, Drama Library, East Asia Library, Economic Growth Center Collection, Engineering and Applied Science Library, Epidemiology and Public Health Library, Film Study Center, Forestry and Environmental Studies Library, Fortunoff Video Archive for Holocaust Testimonies, Geographic Information Systems (GIS) at Yale, Geology Library, Government Documents and Information Center, Historical Sound Recordings, Judaica Collection, Kline Science Library, Latin American Collection, Lewis Walpole Library, Library Shelving Facility, Manuscripts and Archives at SML, Map Collection, Mathematics Library, Medical Historical Library, Microform Reading Room, Mudd Library, Music Library, Near Eastern Collection, Newspaper Reading Room, Nursing Library and Information Resources, Ornithology Library, Slavic and East European Collection, Social Science Libraries and Information Services, Southeast Asia Collection, Special Collections at Yale, Sterling Memorial Library, Visual Resources Collection, Yale Center for British Art, Medical Library, the Law Library. 


\section{ARL Member Libraries as of January 1, 2009}

The Association of Research Libraries (ARL) represents the interests of 123 libraries that serve major North American research institutions. ARL operates as a forum for the exchange of ideas and as an agent for collective action to influence the forces affecting the ability of these libraries to meet the future needs of scholarship. The ARL Statistics and Measurement program is organized around identifying, collecting, analyzing, and distributing quantifiable information describing the characteristics of research libraries. The program offers publications and special member services, and collaborates with other national and international library statistics programs.

\begin{tabular}{|c|c|c|c|}
\hline Institution & Category & Full Name of Institution & Location \\
\hline Alabama & $S$ & University of Alabama & Tuscaloosa, Alabama \\
\hline Alberta & C & University of Alberta & Edmonton, Alberta \\
\hline Arizona & $S$ & University of Arizona & Tucson, Arizona \\
\hline Arizona State & $\mathrm{S}$ & Arizona State University & Tempe, Arizona \\
\hline Auburn & $\mathrm{S}$ & Auburn University & Auburn, Alabama \\
\hline Boston & $\mathrm{P}$ & Boston University & Boston, Massachusetts \\
\hline Boston College & $\mathrm{P}$ & Boston College & Boston, Massachusetts \\
\hline Brigham Young & $\mathrm{P}$ & Brigham Young University & Provo, Utah \\
\hline British Columbia & $\mathrm{C}$ & University of British Columbia & Vancouver, British Columbia \\
\hline Brown & $\mathrm{P}$ & Brown University & Providence, Rhode Island \\
\hline Berkeley, California & $\mathrm{S}$ & University of California, Berkeley & California, Berkeley \\
\hline California, Davis & S & University of California, Davis & Davis, California \\
\hline California, Irvine & S & University of California, Irvine & Irvine, California \\
\hline California, Los Angeles & S & University of California, Los Angeles & Los Angeles, California \\
\hline California, Riverside & S & University of California, Riverside & Riverside, California \\
\hline California, San Diego & S & University of California, San Diego & La Jolla, California \\
\hline California, Santa Barbara & S & University of California, Santa Barbara & Santa Barbara, California \\
\hline Case Western Reserve & $\mathrm{P}$ & Case Western Reserve University & Cleveland, Ohio \\
\hline Chicago & $\mathrm{P}$ & University of Chicago & Chicago, Illinois \\
\hline Cincinnati & S & University of Cincinnati & Cincinnati, Ohio \\
\hline Colorado & S & University of Colorado & Boulder, Colorado \\
\hline Colorado State & S & Colorado State University & Fort Collins, Colorado \\
\hline Columbia & $\mathrm{P}$ & Columbia University & New York, New York \\
\hline Connecticut & S & University of Connecticut & Storrs, Connecticut \\
\hline Cornell & $\mathrm{P}$ & Cornell University & Ithaca, New York \\
\hline Dartmouth & $\mathrm{P}$ & Dartmouth College & Hanover, New Hampshire \\
\hline Delaware & S & University of Delaware & Newark, Delaware \\
\hline Duke & $\mathrm{P}$ & Duke University & Durham, North Carolina \\
\hline Emory & $\mathrm{P}$ & Emory University & Atlanta, Georgia \\
\hline Florida & S & University of Florida & Gainesville, Florida \\
\hline Florida State & $S$ & Florida State University & Tallahassee, Florida \\
\hline George Washington & $\mathrm{P}$ & George Washington University & Washington, DC \\
\hline
\end{tabular}




\begin{tabular}{|c|c|c|c|}
\hline Institution & Category & Full Name of Institution & Location \\
\hline Georgetown & $\mathrm{P}$ & Georgetown University & Washington, DC \\
\hline Georgia & $S$ & University of Georgia & Athens, Georgia \\
\hline Georgia Tech & S & Georgia Institute of Technology & Atlanta, Georgia \\
\hline Guelph & $\mathrm{C}$ & University of Guelph & Guelph, Ontario \\
\hline Harvard & $\mathrm{P}$ & Harvard University & Cambridge, Massachusetts \\
\hline Hawaii & $S$ & University of Hawaii & Honolulu, Hawaii \\
\hline Houston & S & University of Houston & Houston, Texas \\
\hline Howard & $\mathrm{P}$ & Howard University & Washington, DC \\
\hline Illinois, Chicago & S & University of Illinois at Chicago & Chicago, Illinois \\
\hline Illinois, Urbana & S & University of Illinois at Urbana & Urbana, Illinois \\
\hline Indiana & $S$ & Indiana University & Bloomington, Indiana \\
\hline Iowa & $S$ & University of Iowa & Iowa City, Iowa \\
\hline Iowa State & $S$ & Iowa State University & Ames, Iowa \\
\hline Johns Hopkins & $\mathrm{P}$ & Johns Hopkins University & Baltimore, Maryland \\
\hline Kansas & $S$ & University of Kansas & Lawrence, Kansas \\
\hline Kent State & $S$ & Kent State University & Kent, Ohio \\
\hline Kentucky & $S$ & University of Kentucky & Lexington, Kentucky \\
\hline Laval & $\mathrm{C}$ & Laval University & Quebec, Quebec \\
\hline Louisiana State & $S$ & Louisiana State University & Baton Rouge, Louisiana \\
\hline Louisville & $S$ & University of Louisville & Louisville, Kentucky \\
\hline McGill & $\mathrm{C}$ & McGill University & Montreal, Quebec \\
\hline McMaster & $\mathrm{C}$ & McMaster University & Hamilton, Ontario \\
\hline Manitoba & $\mathrm{C}$ & University of Manitoba & Winnipeg, Manitoba \\
\hline Maryland & S & University of Maryland & College Park, Maryland \\
\hline Massachusetts & $S$ & University of Massachusetts & Amherst, Massachusetts \\
\hline MIT & $\mathrm{P}$ & Massachusetts Institute of Technology & Cambridge, Massachusetts \\
\hline Miami & $\mathrm{P}$ & University of Miami & Coral Gables, Florida \\
\hline Michigan & S & University of Michigan & Ann Arbor, Michigan \\
\hline Michigan State & $S$ & Michigan State University & East Lansing, Michigan \\
\hline Minnesota & S & University of Minnesota & Minneapolis, Minnesota \\
\hline Missouri & S & University of Missouri & Columbia, Missouri \\
\hline Montreal & $\mathrm{C}$ & University of Montreal & Montreal, Quebec \\
\hline Nebraska & S & University of Nebraska-Lincoln & Lincoln, Nebraska \\
\hline New Mexico & S & University of New Mexico & Albuquerque, New Mexico \\
\hline New York & $\mathrm{P}$ & New York University & New York, New York \\
\hline North Carolina & S & University of North Carolina & Chapel Hill, North Carolina \\
\hline North Carolina State & S & North Carolina State University & Raleigh, North Carolina \\
\hline Northwestern & $\mathrm{P}$ & Northwestern University & Evanston, Illinois \\
\hline Notre Dame & $\mathrm{P}$ & University of Notre Dame & Notre Dame, Indiana \\
\hline Ohio & $S$ & Ohio University & Athens, Ohio \\
\hline
\end{tabular}




\begin{tabular}{|c|c|c|c|}
\hline Institution & Category & Full Name of Institution & Location \\
\hline Ohio State & $S$ & Ohio State University & Columbus, Ohio \\
\hline Oklahoma & $S$ & University of Oklahoma & Norman, Oklahoma \\
\hline Oklahoma State & S & Oklahoma State University & Stillwater, Oklahoma \\
\hline Oregon & $S$ & University of Oregon & Eugene, Oregon \\
\hline Pennsylvania & $\mathrm{P}$ & University of Pennsylvania & Philadelphia, Pennsylvania \\
\hline Pennsylvania State & S & Pennsylvania State University & University Park, Pennsylvania \\
\hline Pittsburgh & S & University of Pittsburgh & Pittsburgh, Pennsylvania \\
\hline Princeton & $\mathrm{P}$ & Princeton University & Princeton, New Jersey \\
\hline Purdue & S & Purdue University & West Lafayette, Indiana \\
\hline Queen's & $\mathrm{C}$ & Queen's University & Kingston, Ontario \\
\hline Rice & $\mathrm{P}$ & Rice University & Houston, Texas \\
\hline Rochester & $\mathrm{P}$ & University of Rochester & Rochester, New York \\
\hline Rutgers & $S$ & Rutgers University & New Brunswick, New Jersey \\
\hline Saskatchewan & $\mathrm{C}$ & University of Saskatchewan & Saskatoon, Saskatchewan \\
\hline South Carolina & S & University of South Carolina & Columbia, South Carolina \\
\hline Southern California & $\mathrm{P}$ & University of Southern California & Los Angeles, California \\
\hline Southern Illinois & S & Southern Illinois University & Carbondale, Illinois \\
\hline SUNY-Albany & S & University at Albany, State University of New York & Albany, New York \\
\hline SUNY-Buffalo & S & University at Buffalo, State University of New York & Buffalo, New York \\
\hline SUNY-Stony Brook & $\mathrm{S}$ & State University of New York at Stony Brook & Stony Brook, New York \\
\hline Syracuse & $\mathrm{P}$ & Syracuse University & Syracuse, New York \\
\hline Temple & $\mathrm{S}$ & Temple University & Philadelphia, Pennsylvania \\
\hline Tennessee & $\mathrm{S}$ & University of Tennessee & Knoxville, Tennessee \\
\hline Texas & S & University of Texas & Austin, Texas \\
\hline Texas A\&M & S & Texas A\&M University & College Station, Texas \\
\hline Texas Tech & S & Texas Tech University & Lubbock, Texas \\
\hline Toronto & $\mathrm{C}$ & University of Toronto & Toronto, Ontario \\
\hline Tulane & $\mathrm{P}$ & Tulane University & New Orleans, Louisiana \\
\hline Utah & $\mathrm{S}$ & University of Utah & Salt Lake City, Utah \\
\hline Vanderbilt & $\mathrm{P}$ & Vanderbilt University & Nashville, Tennessee \\
\hline Virginia & $\mathrm{S}$ & University of Virginia & Charlottesville, Virginia \\
\hline Virginia Tech & $\mathrm{S}$ & Virginia Polytechnic Institute \& State University & Blacksburg, Virginia \\
\hline Washington & S & University of Washington & Seattle, Washington \\
\hline Washington State & $\mathrm{S}$ & Washington State University & Pullman, Washington \\
\hline Washington U.-St. Louis & $\mathrm{P}$ & Washington University & St. Louis, Missouri \\
\hline Waterloo & C & University of Waterloo & Waterloo, Ontario \\
\hline Wayne State & $\mathrm{S}$ & Wayne State University & Detroit, Michigan \\
\hline Western Ontario & $\mathrm{C}$ & University of Western Ontario & London, Ontario \\
\hline Wisconsin & $\mathrm{S}$ & University of Wisconsin & Madison, Wisconsin \\
\hline Yale & $\mathrm{P}$ & Yale University & New Haven, Connecticut \\
\hline
\end{tabular}




\begin{tabular}{llll} 
Institution & Category & Full Name of Institution & Location \\
\hline York & C & York University & North York, Ontario \\
Boston Public Library & N & Boston Public Library & Boston, Massachusetts \\
Canada Inst. SciTech Info. & X & Canada Inst. for Scientific \& Technical Information & Ottawa, Ontario \\
Center for Research Libs. & N & Center for Research Libraries & Chicago, Illinois \\
Library of Congress & N & Library of Congress & Washington, DC \\
Natl. Agricultural Lib. & N & National Agricultural Library & Beltsville, Maryland \\
Lib. \& Archives of Canada & X & Library and Archives of Canada & Ottawa, Ontario \\
Natl. Library of Medicine & N & National Library of Medicine & Bethesda, Maryland \\
New York Public Library & N & New York Public Library & New York, New York \\
New York State Library & N & New York State Library & Albany, New York \\
Smithsonian Institution & N & Smithsonian Institution & Washington, DC \\
S - U.S. public university & & &
\end{tabular}




\section{Table Numbering Changes 1998-1999 to 1999-2000}

From 1998-1999 to 1999-2000 a number of new tables were added to ARL Annual Salary Survey publication and some old tables were renumbered. As a result of these changes, a new section was added, entitled US ARL University Libraries, which includes Tables 26 and 29. Two new tables showing averages for all US and Canadian institutions were also included as Table 3 and Table 4 . The table below maps the old table-numbering scheme to the new one for purposes of comparison.

\section{Old New}

\section{SAlary LeVels For StAFF IN ARL Libraries}

$1 \quad 1 \quad$ Distribution by Salary Level

$12 \quad$ Salary Trends in ARL University Libraries

N/A 3 Salary Trends in US ARL University Libraries

N/A 4 Salary Trends in Canadian ARL University Libraries

\section{ARL NONUNIVERSITY LIBRARIES}

25 Median and Beginning Professional Salaries in ARL Nonuniversity Libraries

36 Salary Trends in ARL Nonuniversity Libraries

\section{ARL UNIVERSITY LiBRARIES}

47 Filled Positions; Average, Median, Beginning Professional Salaries, And Average Years of Professional Experience in ARL University Libraries, FY 1999-2000

Beginning Professional Salaries in ARL University Libraries; Rank Order Table, FY 1998-1999

Beginning Professional Salaries in ARL University Libraries; Rank Order Table, FY 1999-2000

Median Professional Salaries in ARL University Libraries; Rank Order Table, FY 1998-1999

11 Median Professional Salaries in ARL University Libraries; Rank Order Table, FY 1999-2000

Average Professional Salaries in ARL University Libraries; Rank Order Table, FY 1998-1999

Average Professional Salaries in ARL University Libraries; Rank Order Table, FY 1999-2000

Average, Median, and Beginning Professional Salaries in ARL University Libraries; Summary of Rankings, FYs 1996-1997 to 1999-2000

Distribution of Professional Staff in ARL University Libraries by Salary and Position, FY 1999-2000

14 Distribution of Professional Staff in ARL University Libraries by Salary, Sex, and Position, FY 1999-2000

$15 \quad$ Number and Average Salaries of ARL University Librarians by Position and Sex, FY 1999-2000 


\begin{tabular}{|c|c|c|}
\hline Old & New & \\
\hline 17 & 18 & $\begin{array}{l}\text { Number and Average Years of Experience of ARL University Librarians } \\
\text { by Position and Sex, FY 1999-2000 }\end{array}$ \\
\hline 19 & 19 & $\begin{array}{l}\text { Number and Average Salaries of ARL University Librarians } \\
\text { by Years of Experience and Sex, FY 1999-2000 }\end{array}$ \\
\hline 21 & 20 & Average Salaries of ARL University Librarians by Years of Experience, FY 1999-2000 \\
\hline 22 & 21 & $\begin{array}{l}\text { Number and Average Salaries of ARL University Librarians } \\
\text { by Position and Type of Institution, FY 1999-2000 }\end{array}$ \\
\hline $22 b$ & 22 & $\begin{array}{l}\text { Years of Experience of ARL University Librarians } \\
\text { by Position and Type of Institution, FY 1999-2000 }\end{array}$ \\
\hline 23 & 23 & $\begin{array}{l}\text { Number and Average Salaries of ARL University Librarians } \\
\text { by Position and Size of Professional Staff, FY 1999-2000 }\end{array}$ \\
\hline $23 b$ & 24 & $\begin{array}{l}\text { Years of Experience of ARL University Librarians } \\
\text { by Position and Size of Professional Staff, FY 1999-2000 }\end{array}$ \\
\hline 24 & 25 & $\begin{array}{l}\text { Average Salaries of ARL University Librarians } \\
\text { by Position and Geographic Region, FY 1999-2000 }\end{array}$ \\
\hline
\end{tabular}

\section{US ARL UNIVERSITY LIBRARIES}

N/A $26 \quad$ Average Salaries of US ARL University Librarians

by Position and Years of Experience, FY 1999-2000

\section{US ARL UNIVERSITY LIBRARIES}

$16 \quad 27 \quad$ Number and Average Salaries of Minority US ARL University Librarians by Position and Sex, FY 1999-2000

$18 \quad 28 \quad$ Number and Average Years of Experience of Minority US ARL University Librarians by Position and Sex, FY 1999-2000

N/A $29 \quad$ Number and Average Salaries of US ARL University Librarians by Years of Experience and Sex, FY 1999-2000

$20 \quad 30 \quad$ Number and Average Salaries of Minority US ARL University Librarians by Years of Experience and Sex, FY 1999-2000

\section{Canadian ARL University Libraries}

$2531 \quad$ Filled Positions; Average, Median, and Beginning Professional Salaries; and Average Years of Professional Experience in Canadian ARL University Libraries, FY 1999-2000

$26 \quad 32 \quad$ Number and Average Salaries of Canadian ARL University Librarians by Position and Sex, FY 1999-2000

$27 \quad 33 \quad$ Number and Average Years of Experience of Canadian ARL University Librarians by Position and Sex, FY 1999-2000

$28 \quad 34 \quad$ Number and Average Salaries of Canadian ARL University Librarians by Years of Experience and Sex, FY 1999-2000 


\section{ARL University Medical Libraries}

$29 \quad 35$

Filled Positions; Average, Median, Beginning Professional Salaries; and Average Years of Professional Experience in ARL University Medical Libraries, FY 1999-2000

Beginning Professional Salaries in ARL University Medical Libraries;

Rank Order Table, FY 1999-2000

$31 \quad 37$

Median Professional Salaries in ARL University Medical Libraries;

Rank Order Table, FY 1999-2000

Average Professional Salaries in ARL University Medical Libraries;

Rank Order Table, FY 1999-2000

Number and Average Salaries of ARL University Medical Librarians by Position and Sex, FY 1999-2000

Number and Average Years of Experience of ARL University Medical Librarians by Position and Sex, FY 1999-2000

Number and Average Salaries of ARL University Medical Librarians

by Years of Experience and Sex, FY 1999-2000

\section{ARL University LAW LibraRIES}

Filled Positions; Average, Median, Beginning Professional Salaries; and Average Years of Experience in ARL University Law Libraries, FY 1999-2000

Beginning Professional Salaries in ARL University Law Libraries; Rank Order Table, FY 1999-2000

Median Professional Salaries in ARL University Law Libraries; Rank Order Table, FY 1999-2000

Average Professional Salaries in ARL University Law Libraries; Rank Order Table, FY 1999-2000

Number and Average Salaries of ARL University Law Librarians

by Position and Sex, FY 1999-2000

$41 \quad 47$

Number and Average Years of Experience of ARL University Law Librarians by Position and Sex, FY 1999-2000

Number and Average Salaries of ARL University Law Librarians

by Years of Experience and Sex, FY 1999-2000 
